\title{
INTRINSIC DISTANCES, MEASURES AND GEOMETRIC FUNCTION THEORY ${ }^{1}$
}

\author{
BY SHOSHICHI KOBAYASHI
}

To the memory of Carl B. Allendoerfer

\section{Table of Contents}

1. Introduction

2. Intrinsic pseudo-distances $\quad 359$

3. Hyperbolic complex spaces 364

4. Metric and holomorphic completeness $\quad 367$

5. Intrinsic volume forms

6. Schwarz lemma and differential geometric criteria for hyperbolicity

7. Ample canonical bundles and manifolds of general type 374

8. Families of holomorphic and meromorphic maps 377

9. Automorphisms and endomorphisms of complex spaces 383

10. Extension of holomorphic and meromorphic maps 386

11. Defect relations $\quad 387$

12. Examples and applications $\quad 390$

13. Unsolved problems $\quad 399$

1. Introduction. In 1879, Picard discovered the following spectacular theorems:

The Little Picard Theorem. If a function $f(z)$ holomorphic in $|z|<\infty$ misses two values, say 0 and 1 , then $f(z)$ is constant.

The Great Picard Theorem. If a function $f(z)$ holomorphic in $0<|z|<r$ misses two values, say 0 and 1 , then $z=0$ is either a removable singularity or a pole.

The original proofs of these theorems involved the use of the modular function $\lambda: H \rightarrow \boldsymbol{C}-\{0,1\}$, where $H$ denotes the upper half-plane $\{z=x+i y ; y>0\}$. Subsequent successful efforts by E. Borel, Landau, Schottky, Montel, Bloch and others to find so-called "elementary" proofs (i.e., proofs free of the modular function) greatly enriched the theory of functions.

In his epoch making paper of $1925, \mathrm{R}$. Nevanlinna established the theory of value distributions. The so-called defect relation for a meromorphic

An expanded version of an invited address delivered before the Annual Meeting of the Society in San Francisco on January 15, 1974; received July 16, 1975.

AMS (MOS) subject classifications (1970). Primary 32H15, 32H20, 32H25; Secondary 32A15, 32A17, 32C10, 32E10, 32F15, 32H10, 32J05, 32J25, 32M05, 30A70, 14E05, 53C55.

${ }^{1}$ Partially supported by NSF Grant GP-42020X. 
function $f(z),|z|<\infty$ (i.e., a holomorphic map $\left.f: \boldsymbol{C} \rightarrow P_{1}(\boldsymbol{C})\right)$ states that if $f$ is not a constant, then

$$
\sum_{a \in P_{1}(C)} \delta(f, a) \leqq 2,
$$

where $\delta(f, a)$ is the defect of the value $a$. Without giving the definition of the defect $\delta(f, a)$, we say simply that $\delta(f, a)$ is a number between 0 and 1 which measures how frequently the value $a$ is taken (or rather not taken) by the function $f$. Thus, $\delta(f, a)=1$ if $f$ misses the value a completely while $\delta(f, a)=0$ if $f$ takes the value $a$ as often as any other value. If $f$ misses three values, say $\infty, 0$ and 1 , then $\delta(f, \infty)=\delta(f, 0)=\delta(f, 1)=1$, and the defect relation implies that $f$ is a constant. It may be said that the theory of Nevanlinna is concerned with the quantitative measurement of the value distribution of $f$ while the earlier results of Picard and others are qualitative in nature.

As early as in 1897, E. Borel generalized the little Picard theorem to holomorphic maps of $\boldsymbol{C}$ into the complement of $n+2$ hyperplanes $H_{j}$, $j=0,1, \cdots, n+1$, in the projective space $P_{n}(\boldsymbol{C})$. Subsequently, Bloch in 1926 and H. Cartan in 1928 obtained more precise results by considering holomorphic maps of the unit disk into $P_{n}(\boldsymbol{C})-\bigcup H_{j}$. On the other hand, Nevanlinna's quantitative results have been also extended to holomorphic maps of $\boldsymbol{C}$ into $P_{n}(\boldsymbol{C})$; the value distribution theory for holomorphic curves in $P_{n}(\boldsymbol{C})$ were initiated by $\mathrm{H}$. and J. Weyl in 1938 and were essentially completed by Ahlfors in 1941. In the meantime, it has become increasingly clear owing to a series of Ahlfors' papers in 1929-1936 and more recent papers of Chern and others that one gets the best view of the subject by looking from the differential geometric standpoint.

This paper is a report on qualitative results in the higher dimensional value distribution theory which can be best described in terms of certain intrinsic metrics and measures. We shall make a few brief comments on quantitative results in the last section of the paper.

To explain the content of the paper further, a good place to start is Ahlfors' paper of 1938 [1] in which he uncovers the differential geometric character of the Schwarz lemma. Let $D$ be the unit disk with the Poincare metric $d s^{2}$ of Gaussian curvature -1 and let $X$ be a Riemann surface with a hermitian metric $d s_{X}^{2}$ of Gaussian curvature $\leqq-1$. Then every holomorphic map $f: D \rightarrow X$ is distance-decreasing, i.e., $f^{*} d s_{X}^{2} \leqq d s^{2}$ on $D$. When $X=D$ and $d s_{X}^{2}=d s^{2}$, this is nothing but the classical Schwarz-Pick lemma. It follows easily that for such a Riemann surface $X$ every holomorphic map $f: C \rightarrow X$ is a constant. Thus an "elementary" proof of the little Picard theorem may be obtained by constructing a metric $d s_{X}^{2}$ of curvature $\leqq-1$ on $X=C-\{0,1\}$. (On the other hand, if we make use of the modular function $\lambda$, we get a metric of curvature precisely equal to -1 on $\boldsymbol{X}=\boldsymbol{C}-\{0,1\}$ from the Poincaré metric of the upper half-plane $H$.) Viewing the Schwarz-Pick-Ahlfors lemma from a different angle, we consider all pseudo-distances on a Riemann surface or, more generally, a complex space $X$ which makes every 
holomorphic map $f: D \rightarrow X$ distance-decreasing and take the largest one. Then we ask if this intrinsically defined pseudo-distance $d_{X}$ is actually a distance. Then the result of Ahlfors can be viewed as a theorem providing a differential geometric sufficient condition for $d_{X}$ to be a distance. We say that a complex space $X$ is (complete) hyperbolic if $d_{X}$ is a (complete) distance. Corresponding to the generalized Schwarz-Pick lemma for holomorphic maps from a polydisk into a complex manifold of the same dimension, we can introduce an analogous intrinsic measure or pseudovolume form $\Psi_{X}$ on a complex manifold $X$. There are a number of general results on the pseudo-distance $d_{\mathrm{X}}$ and the pseudo-volume form $\Psi_{\mathrm{X}}$. But deeper and more interesting problems seem to be those of determining or, at least, estimating $d_{X}$ and $\Psi_{X}$ for individual $X$. While the pseudo-volume form $\Psi_{X}$ is positive everywhere for a large class of complex manifolds, the pseudo-distance $d_{X}$ is often partially degenerate, and it is important to determine where $d_{x}$ degenerates.

Beyond immediate applications to geometric function theory of several complex variables, in particular, to generalized Picard theorems, $d_{X}$ and $\Psi_{X}$ have found their ways into algebraic manifolds (particularly of general type), Teichmüller spaces and higher dimensional Diophantine geometry.

Since much of general basic results are in my monograph Hyperbolic manifolds and holomorphic mappings, our emphasis here is on recent progress, examples and unsolved problems. Examples represent the most important aspect of the theory.

I tried to assemble a reasonably complete bibliography on all work done on hyperbolic manifolds and related subjects, supplementing the bibliography of my monograph; the list of about 190 papers and books in this report contains approximately 50 of the 133 articles mentioned in my monograph. But I fear, because of a large number of papers involved, I overlooked quite a few. Since our main interests are in higher dimensional complex manifolds, papers on functions of one variable are completely disregarded unless they have a direct bearing on our theory. Results in Nevanlinna theory are mentioned only in passing and references on this subject are far from being complete even in the higher dimensional case.

During the preparation of this report, I had numerous useful conversations with S. S. Roan on algebraic geometric aspects of the theory.

2. Intrinsic pseudo-distances. Let $D$ denote the unit disk $\{z \in \boldsymbol{C} ;|z|<1\}$ in $\boldsymbol{C}$ with Poincaré metric

$$
d s^{2}=4 d z d \bar{z} /\left(1-|z|^{2}\right)^{2}
$$

of Gaussian curvature -1 . Let $\rho$ denote the distance function defined by $d s^{2}$. Then the classical lemma of Schwarz, reformulated geometrically by Pick, states:

THEOREM 2.1. Every holomorphic map $f: D \rightarrow D$ is distance-decreasing, that is, $f^{*} d s^{2} \leqq d s^{2}$, or equivalently,

$$
\rho\left(f(z), f\left(z^{\prime}\right)\right) \leqq \rho\left(z, z^{\prime}\right) \quad \text { for } z, z^{\prime} \in D .
$$


This is perhaps the most basic result in the geometric theory of functions. Carathéodory [1], [2] was the first to generalize the Schwarz-Pick lemma to holomorphic maps between higher dimensional domains. Given a complex space $X$, let $\operatorname{Hol}(X, D)$ denote the family of holomorphic maps $f: X \rightarrow D$. He defined a pseudo-distance $c_{X}$ on $X$ by

$$
c_{X}(p, q)=\sup _{f} \rho(f(p), f(q)) \text { for } p, q \in X
$$

where the supremum is taken over all $f \in H(X, D)$. (An argument using normal families shows that this supremum is actually achieved by a certain map $f$.) Carathéodory was primarily interested in bounded domains in $\boldsymbol{C}^{n}$, for which his pseudo-distance is a bona fide distance. But $c_{X}(p, q)$ can be, in general, zero even when $p$ and $q$ are distinct. In fact, $c_{X}$ vanishes identically if $X=\boldsymbol{C}$ (Liouville's theorem) or if $X$ is compact (the maximal principle). From the definition of the Carathéodory pseudo-distance it follows immediately that if $X$ and $Y$ are two complex spaces, then

$$
c_{Y}(f(p), f(q)) \leqq c_{X}(p, q) \text { for } f \in \operatorname{Hol}(X, Y), p, q \in X,
$$

that is, $f: X \rightarrow Y$ is distance-decreasing. The fact that the Carathéodory pseudo-distance generalizes the Poincaré distance $\rho$, i.e.,

$$
c_{D}=\rho
$$

is easily seen to be essentially equivalent to the Schwarz-Pick lemma.

In studying the Carathéodory pseudo-distance, it is convenient to consider also its infinitesimal form. We define

$$
E_{X}(v)=\sup _{f}\left\|f_{*}(v)\right\| \text { for } v \in T(X),
$$

where $\left\|f_{*}(v)\right\|$ is the length of the tangent vector $f_{*}(v)$ of $D$ measured by the Poincaré metric $d s^{2}$ and the supremum is taken over all $f \in \operatorname{Hol}(X, D)$. Corresponding to (2.3) and (2.4), we have

$$
E_{Y}\left(f_{*}(v)\right) \leqq E_{X}(v) \text { for } f \in \operatorname{Hol}(X, Y), v \in T(X),
$$

and

$$
E_{D}^{2}=d s^{2} .
$$

Shortly after Carathéodory, another intrinsic metric was introduced by Bergman [1]. He was also mainly interested in bounded domains in $\boldsymbol{C}^{n}$. Adapting his construction to a complex manifold $X$, let $H$ be the Hilbert space of square-integrable holomorphic $n$-forms $\omega$ on $X($ where $n=\operatorname{dim} X)$ :

$$
H=\left\{\text { holomorphic } n \text {-forms } \omega ; \int_{X}(\sqrt{ }-1)^{n^{2}} \omega \wedge \bar{\omega}<\infty\right\} .
$$

Using a complete orthonormal basis $\omega_{0}, \omega_{1}, \cdots$ for $H$, we define the Bergman kernel form by

$$
B_{X}=\sum_{j=0}^{\infty}(\sqrt{ }-1)^{n^{2}} \omega_{j} \wedge \bar{\omega}_{j} .
$$

In general, $B_{X}$ is a pseudo-volume form, that is, it might vanish at some 
point (or even identically). If $B_{X}$ is strictly positive everywhere, we set

$$
d s_{X}^{2}=2 \sum g_{j \bar{k}} d z^{j} d \bar{z}^{k} \quad \text { with } g_{j \bar{k}}=\partial^{2} \log B / \partial z^{j} \partial \bar{z}^{k},
$$

where $B$ is the coefficient of $B_{X}$ with respect to a local coordinate system $z^{1}, \cdots, z^{n}$ of $X$, i.e.,

$$
B_{X}=(\sqrt{ }-1)^{n^{2}} B d z^{1} \wedge \cdots \wedge d z^{n} \wedge d \bar{z}^{1} \wedge \cdots \wedge d \bar{z}^{n} .
$$

In general, $d s_{X}^{2}$ is positive semi-definite. When $H$ is very ample in a suitable sense, $d s_{X}^{2}$ is positive definite and is a Kähler metric on $X$. Then it is called the Bergman metric of $X$. In particular, when $X$ is a bounded domain in $\boldsymbol{C}^{n}$, $d s_{X}^{2}$ is positive definite.

Finally, we introduce another intrinsic pseudo-distance $d_{x}$, which will be the main subject of this paper. Given two points $p, q$ of a complex space $X$, we consider a chain of holomorphic disks from $p$ to $q$, that is, a chain of points $p=p_{0}, p_{1}, \cdots, p_{k}=q$ of $X$, pairs of points $a_{1}, b_{1}, \cdots, a_{k}, b_{k}$ of $D$ and holomorphic maps $f_{1}, \cdots, f_{k} \in \operatorname{Hol}(D, X)$ such that

$$
f_{i}\left(a_{i}\right)=p_{i-1} \quad \text { and } \quad f_{i}\left(b_{i}\right)=p_{i} \quad \text { for } i=1, \cdots, k .
$$

The length of this chain is defined to be

$$
\rho\left(a_{1}, b_{1}\right)+\cdots+\rho\left(a_{k}, b_{k}\right) .
$$

The distance $d_{X}(p, q)$ is given by

$$
d_{X}(p, q)=\inf \left(\rho\left(a_{1}, b_{1}\right)+\cdots+\rho\left(a_{k}, b_{k}\right)\right),
$$

where the infimum is taken over all chains of holomorphic disks from $p$ to $q$. As in the case of the Carathéodory pseudo-distance, $d_{X}(p, q)$ can be zero even when $p$ and $q$ are distinct. In fact, it is easy to verify that

$$
d_{\mathbf{c}}(p, q)=0, \quad p, q \in \boldsymbol{C} .
$$

From definition (2.14) we see that $d_{X}$ is defined in a manner "dual" to $c_{X}$. The following construction of the infinitesimal form of $d_{X}$ will make this duality more apparent. We define (at least when $X$ is nonsingular)

$$
F_{X}(v)=\inf _{f}\left\{\|u\| ; u \in T(D) \text { and } f_{*}(u)=v\right\} \text { for } v \in T(X),
$$

where $\|u\|$ denotes the length of the tangent vector $u$ measured by the Poincaré metric $d s^{2}$ of $D$ and the infimum is taken over all $f \in \operatorname{Hol}(D, X)$ and $u \in T(D)$ such that $f_{*}(u)=v$. Corresponding to $(2.3),(2.4),(2.6)$ and (2.7), we obtain immediately

$$
\begin{gathered}
d_{Y}(f(p), f(q)) \leqq d_{X}(p, q) \quad \text { for } f \in \operatorname{Hol}(X, Y), p, q \in X ; \\
d_{D}=\rho ; \\
F_{Y}\left(f_{*}(v)\right) \leqq F_{X}(v) \quad \text { for } f \in \operatorname{Hol}(X, Y), v \in T(X) ; \\
\\
F_{D}^{2}=d s^{2} .
\end{gathered}
$$

We have thus constructed three (pseudo-)distances $d s_{X}^{2}, c_{X}$ and $d_{X}$, which are all intrinsic invariants of the complex structure of $X$. They all generalize 
the Poincare metric of the unit disk. The Bergman metric $d s_{X}^{2}$ has the advantage of being a Kähler metric while $c_{X}$ and $d_{X}$ are not very smooth in general. On the other hand, both $c_{X}$ and $d_{x}$ have the advantage of being defined on any complex space $X$ (singular or nonsingular, finite or infinite dimensional), albeit degenerate in some cases while $d s_{X}^{2}$ can be defined only for a limited class of complex manifolds. Moreover, the distance-decreasing properties (2.3) and (2.17) make $c_{\mathrm{X}}$ and $d_{\mathrm{Y}}$ particularly useful in studying holomorphic maps. They are the two extreme pseudo-distances with respect to this property in the following sense:

THEOREM 2.2. Let $X$ be a complex space.

(1) If $\delta_{X}$ is a pseudo-distance such that $\rho(f(p), f(q)) \leqq \delta_{X}(p, q)$ for $p, q \in X$ and $f \in \operatorname{Hol}(X, D)$, then $c_{X}(p, q) \leqq \delta_{X}(p, q)$ for all $p, q \in X$;

(2) If $\delta_{X}$ is a pseudo-distance such that $\delta_{X}(f(a), f(b)) \leqq \rho(a, b)$ for $a, b \in D$ and $f \in \operatorname{Hol}(D, X)$, then $\delta_{X}(p, q) \leqq d_{X}(p, q)$ for all $p, q \in X$.

This theorem characterizes both $c_{\mathrm{X}}$ and $d_{\mathrm{X}}$ and can be used to define them. In particular, we have

$$
c_{X}(p, q) \leqq d_{X}(p, q) \text { for } p, q \in X .
$$

A little more generally, suppose we are given a family $\mathscr{C}$ of complex spaces of which the unit disk $D$ is a member and a pseudo-distance $\delta_{X}$ for each member $X$ of the family $\mathscr{C}$ such that $\delta_{D}=\rho$ and

$$
\delta_{Y}(f(p), f(q)) \leqq \delta_{X}(p, q) \quad \text { for } p, q \in X \in \mathscr{C}, Y \in \mathscr{C}, f \in \operatorname{Hol}(X, Y)
$$

then

$$
c_{X} \leqq \delta_{X} \leqq d_{X} \quad \text { for } X \in \mathscr{C} .
$$

The intrinsic pseudo-distance introduced by Chern, Levine and Nirenberg [1] is such a pseudo-distance and hence lies between $c_{X}$ and $d_{X}$.

If $X$ and $Y$ are complex manifolds for which the Bergman metrics $d s_{X}^{2}$ and $d s_{Y}^{2}$ exist, then the Bergman metric exists for $X \times Y$ and it is given by $d s_{X}^{2}+d s_{Y}^{2}$. Thus $X \times Y$ is the Kählerian product of $X$ and $Y$. On the other hand, we have (see Royden [1])

THeORem 2.3. For any two complex spaces $X$ and $Y$, we have

(1) $c_{X \times Y}\left((p, q),\left(p^{\prime}, q^{\prime}\right)\right)=\operatorname{Max}\left\{c_{X}\left(p, p^{\prime}\right), c_{Y}\left(q, q^{\prime}\right)\right\} \quad$ for $p, p^{\prime} \in X, q, q^{\prime} \in Y$;

(2) $d_{X \times Y}\left((p, q),\left(p^{\prime}, q^{\prime}\right)\right)=\operatorname{Max}\left\{d_{X}\left(p, p^{\prime}\right), d_{Y}\left(q, q^{\prime}\right)\right\} \quad$ for $p, p^{\prime} \in X, q, q^{\prime} \in Y$.

For the corresponding infinitesimal pseudo-metrics, we have

$$
\begin{array}{ll}
E_{X \times Y}(u, v)=\operatorname{Max}\left\{E_{X}(u), E_{Y}(v)\right\} & \text { for } u \in T(X), v \in T(Y) ; \\
F_{X \times Y}(u, v)=\operatorname{Max}\left\{F_{X}(u), F_{Y}(v)\right\} & \text { for } u \in T(X), v \in T(Y) .
\end{array}
$$

This theorem implies that $c_{X \times Y}$ and $d_{X \times Y}$ cannot be smooth except in the trivial case.

Let $X^{\prime}$ be a (not necessarily closed) complex subspace of an arbitrary complex subspace $X$. From the distance-decreasing property of the injection 
$X^{\prime} \rightarrow X$, we obtain

$$
c_{X^{\prime}} \geqq c_{X} \quad \text { and } \quad d_{X^{\prime}} \geqq d_{X} \quad \text { on } X^{\prime} .
$$

But we have

Theorem 2.4. (1) If $X$ is normal and $A=X-X^{\prime}$ is contained in an analytic subset of codimension at least 1 in $X$, then $c_{X^{\prime}}=c_{X}$ on $X^{\prime}$;

(2) If $X$ is a complex manifold whose tangent bundle is spanned by its global holomorphic sections and if $A=X-X^{\prime}$ is contained in an analytic subset of codimension at least 2 , then $d_{\mathrm{X}^{\prime}}=d_{\mathrm{X}}$ on $X^{\prime}{ }^{2}$

(3) If $X$ is a complex manifold with the Bergman metric $d s_{X}^{2}$ and if $A=X-X^{\prime}$ is contained in an analytic subset of codimension at least 1 , then $d s_{X^{\prime}}^{2}=d s_{X}^{2}$ on $X^{\prime}$.

(1) follows from Riemann's extension theorem. (2) is in Campbell and Ogawa [1] and is applicable to a domain in $\boldsymbol{C}^{n}$. (3) is in Bremermann [1]. Generally, $d_{x}$ is more sensitive to the removal of analytic subsets.

Perhaps the most striking difference between $c_{X}$ and $d_{X}$ is given by

THEOREM 2.5. Let $\tilde{X}$ be a covering space of a complex space $X$ with projection $\pi: \tilde{X} \rightarrow X$. Then

$$
d_{X}(p, q)=d_{\tilde{X}}\left(\pi^{-1}(p), \pi^{-1}(q)\right) \quad \text { for } p, q \in X .
$$

Infinitesimally, we have $F_{\tilde{X}}=\pi^{*} F_{\mathrm{X}}$.

This follows from the fact that every map $f: D \rightarrow X$ lifts to a map $f: D \rightarrow \tilde{X}$. The theorem fails completely for $c_{X}$ and $d s_{X}^{2}$.

The concept of an inner distance of Rinow [1] will clarify some points concerning $c_{X}, d_{X}, E_{X}$ and $F_{X}$. In general, let $X$ be a topological space with a pseudo-distance function $d$. Given a curve $\gamma(t), a \leqq t \leqq b$, in $X$, its length $L(\gamma)$ is defined by

$$
L(\gamma)=\sup \sum d\left(\gamma\left(t_{i-1}\right), \gamma\left(t_{i}\right)\right) \text {, }
$$

where the supremum is taken over all subdivisions $a=t_{0}<t_{1}<\cdots<t_{k}=b$ of the interval $[a, b]$. A curve $\gamma$ is said to be rectifiable if its length is finite. Assume that $X$ is finitely arcwise connected in the sense that every pair of points $x, y$ of $X$ can be joined by a rectifiable curve. Then we can define a new pseudo-distance $d^{i}$, called the inner distance induced by $d$, by setting

$$
d^{i}(x, y)=\inf L(\gamma)
$$

where the infimum is taken over all rectifiable curves from $x$ to $y$. It follows immediately that $d \leqq d^{i}$ but that the arc-length $L^{i}$ defined by $d^{i}$ coincides with $L$. We say that $d$ is inner if $d=d^{i}$. The terminology is consistent since $d^{i}$ is inner, i.e., $d^{i}=\left(d^{i}\right)^{i}$.

Now we can state

\footnotetext{
${ }^{2}$ According to Howard and Ochiai, the first condition is not necessary. This implies Theorem 3.11 .
} 
TheORem 2.6. Let $X$ be a complex space. Then

(1) The pseudo-metric $F_{X}$ is upper-semicontinuous on $T(X)$, and the pseudo-distance $d_{X}$ is the integrated form of $F_{X}$ and is inner.

(2) The pseudo-metric $E_{X}$ is continuous on $T(X)$, and the inner pseudodistance $c_{X}^{i}$ induced by $c_{X}$ is the integrated form of $E_{X}$.

For the proof of (1), see Royden [1]. The fact that $d_{X}$ is inner can be directly proved rather easily (Kobayashi [5]). For (2), see Reiffen [1]. According to T. Barth, $c_{X}$ is, in general, not inner.

Both $E_{X}$ and $F_{X}$ are differential pseudo-metrics in the sense of Grauert and Reckziegel [1] or complex Finsler pseudo-metrics in a very general sense. In general, a differential pseudo-metric $G$ on $X$ is a nonnegative function on $T(X)$ such that

$$
G(\lambda v)=|\lambda| \cdot G(v) \text { for } v \in T(X), \lambda \in \mathbf{C} .
$$

For the sake of simplicity, assume that $X$ is a complex manifold. Then it is sometimes convenient to write

$$
G=G(z, \zeta)
$$

in terms of a local coordinate system $z=\left(z^{1}, \cdots, z^{n}\right)$ of $X$ and the induced fibre coordinates $\zeta=\left(\zeta^{1}, \cdots, \zeta^{n}\right)$ of $T(X)$. If $G$ is twice differentiable outside of the zero section $\zeta=0$ of $T(X)$, then

$$
\begin{aligned}
G^{2}(z, \zeta) & =\frac{\partial^{2}}{\partial \lambda \partial \bar{\lambda}}\left(\lambda \bar{\lambda} G^{2}(z, \zeta)\right)=\frac{\partial^{2}}{\partial \lambda \partial \bar{\lambda}}\left(G^{2}(z, \lambda \zeta)\right) \\
& =\sum G_{\alpha \bar{\beta}}(z, \zeta) \zeta^{\alpha} \bar{\zeta}^{\beta}, \text { where } G_{\alpha \bar{\beta}}=\frac{\partial^{2}\left(G^{2}(z, \zeta)\right)}{\partial \zeta^{\alpha} \partial \bar{\zeta}^{\beta}} .
\end{aligned}
$$

If $\left(G_{\alpha \bar{\beta}}\right)$ is positive definite, then $G$ is said to be convex and the natural connection can be defined (see Kobayashi [7]). Even when $G$ is not smooth, its holomorphic sectional curvature can still be defined so long as $G$ is positive. Given a 1-dimensional complex subspace $\sigma$ of $T_{x}(X)$, let $S$ be a piece of complex submanifold through $x$ such that $\sigma=T_{x}(S)$. Restricting the metric $G$ to the surface $S$, let $k_{S}(x)$ be the Gaussian curvature of $S$ at $x$; this can be defined even when the (Riemannian) metric $G$ is not smooth (see, for example, Rinow [1] and Reckziegel [1]). The homomorphic sectional curvature $k_{\mathrm{X}}(\sigma)$ for $\sigma$ is by definition (cf. Grauert-Reckziegel [1])

$$
k_{X}(\sigma)=\sup k_{S}(x)
$$

where the supremum is taken over all $S$ such that $\sigma=T_{x}(S)$.

All this applies to $E_{X}$ and $F_{X}$, but very little differential geometric study has been made of $E_{X}$ and $F_{X}$ except for the paper of Reiffen [1] where the Finsler structure of $E_{X}$ was investigated systematically.

3. Hyperbolic complex spaces. We are naturally interested in complex spaces $X$ with nontrivial $d_{X}$. We say that $X$ is hyperbolic if $d_{X}$ is a distance, i.e., $d_{X}(p, q)>0$ whenever $p \neq q$. If $X$ is hyperbolic, the topology induced by $d_{\mathrm{X}}$ coincides with the complex space topology of $X$ (Barth [1] and Royden 
[1]). The hyperbolicity condition, when expressed in terms of the infinitesimal metric $F_{X}$, is slightly stronger than $F_{X}(v)>0$ for all nonzero $v \in T(X)$ (see Royden [1]). A hyperbolic space $X$ is complete if it is Cauchy complete with respect to $d_{x}$. Then we have

THEOREM 3.1. If $X$ is a hyperbolic complex space, it is complete when and only when every closed bounded subset of $X$ is compact.

This follows from the fact that $d_{\mathrm{X}}$ is an inner distance (see $\S 2$ ) and from the following general result (Rinow [1, p. 172]).

LEMMA. A locally compact metric space $X$ with an inner distance $d$ is Cauchy complete if and only if every closed bounded subset of $X$ is compact.

As immediate consequences of Theorems 2.3 and 2.5 , we have

THEOREM 3.2. If $X$ and $Y$ are both (complete) hyperbolic, so is $X \times Y$.

THEOREM 3.3. Let $\tilde{X}$ be a covering space of $X$. Then $\tilde{X}$ is (complete) hyperbolic if and only if $X$ is.

The following related result can be directly and easily proved (cf. Reckziegel [1], Kaup [1], Kwack [1]).

THEOREM 3.4. (1) Let $\pi: \tilde{X} \rightarrow X$ be a holomorphic map such that $\pi^{-1}(x)$ is discrete for each $x \in X$. If $X$ is hyperbolic, so is $\tilde{X}$.

(2) Let $\pi: \tilde{X} \rightarrow X$ be a proper holomorphic map such that $\pi^{-1}(x)$ is discrete (and hence finite) for each $x \in X$. If $X$ is complete hyperbolic, so is $\tilde{X}$.

(3) If $\tilde{X}$ is the normalization of a (complete) hyperbolic space $X$, it is also (complete) hyperbolic.

We shall be interested in complex spaces $X$ for which $d_{X}$ is only partially degenerate since such spaces are more common than hyperbolic spaces. We say that $X$ is hyperbolic modulo a subset $\Delta$ if $d_{X}(p, q)>0$ unless $p=q$ or $p, q \in \Delta$. In applications, the subset $\Delta$ is usually an analytic subset of $X$. A complex space $X$ is said to be complete hyperbolic modulo $\Delta$ if it is hyperbolic modulo $\Delta$ and if for every Cauchy sequence $\left\{p_{n}\right\}$ in $X$ with respect to the pseudo-distance $d_{\mathrm{x}}$ we have one of the following possibilities:

(a) $\left\{p_{n}\right\}$ converges to a point $p$ of $X$;

(b) For every open neighborhood $U$ of $\Delta$ in $X$, there exists an integer $n_{0}$ such that $p_{n} \in U$ for $n \geqq n_{0}$.

This is probably a good place to state the principle of the little Picard theorem:

TheOREM 3.5. Let $X$ be a complex space for which $d_{X} \equiv 0$. If $Y$ is hyperbolic, then every holomorphic map $f: X \rightarrow Y$ is a constant map. More generally, if $Y$ is hyperbolic modulo a subset $\Delta$, then every holomorphic map $f: X \rightarrow Y$ is either constant or $f(X) \subset \Delta$.

This is a trivial consequence of the fact that $f$ is distance-decreasing.

To state the principle of the great Picard theorem, we have to introduce another concept. Let $Y$ be a relatively compact, open subset of a complex 
space $Z$, and $\Delta$ a (possibly empty) subset of $Z$. Then $Y$ is said to be hyperbolically imbedded in $Z$ modulo $\Delta$ if

(a) $Y$ is hyperbolic modulo $\Delta \cap Y$;

(b) For every point $p$ of $\partial Y(=\bar{Y}-Y)$ not contained in $\Delta$ and every neighborhood $U$ of $p$ in $Z$, there is a smaller neighborhood $V$ such that $d_{Y}(V \cap Y, Y-U)>0$. We say that $Y$ is hyperbolically imbedded in $Z$ if it is so modulo the empty set $\Delta$. This concept will be essential in discussing a generalization of the theorem of Montel on normal families.

The principle of the great Picard theorem states:

THEOREM 3.6. Let $X$ be a complex manifold and A a complex subspace whose singularities are normal crossings. Let $Y$ be hyperbolically imbedded in a complex space $Z$. Then every holomorphic map $f: X-A \rightarrow Y$ extends to a holomorphic map $f: X \rightarrow Z$.

The condition on $A$ means that, locally, $X=D^{n}$ and $X-A=D^{* k} \times D^{n-k}$ for some $k$, where $D^{*}=D-\{0\}$. Making use of Hironaka's theorem on resolution of singularities, we obtain

Corollary 3.7. Let $X$ be a complex space and A a complex subspace. Let $Y$ be hyperbolically imbedded in $Z$. Then every meromorphic map $f: X-A \rightarrow$ $Y$ extends to a meromorphic map $f: X \rightarrow Z$.

If $Y$ is already compact, then the restriction on $A$ can be removed.

Corollary 3.8. Let $X$ be a complex manifold and A a complex subspace. Let $Y$ be a compact hyperbolic space. Then every holomorphic map $f: X-A \rightarrow$ $Y$ extends to a holomorphic map $f: X \rightarrow Y$.

COROllary 3.9. Let $X$ be a complex manifold and $Y$ a compact hyperbolic space. Then every meromorphic map $f: X \rightarrow Y$ is necessarily holomorphic.

Corollary 3.10. Let $X$ be a complex space and A a complex subspace. Let $Y$ be a compact hyperbolic space. Then every meromorphic map $f: X-A \rightarrow Y$ extends to a meromorphic map $f: X \rightarrow Y$.

Corollary 3.8 was first proved by Kwack [1] and then it was generalized to Theorem 3.6 by Kobayashi [4] when $A$ is nonsingular and by Kiernan [3] in full. The special case of $\operatorname{dim} X=\operatorname{dim} Y=\operatorname{dim} Z=1$ is in Huber [1, Satz 2]. It is not clear what type of theorem we can expect when $Y$ is hyperbolically imbedded in $Z$ modulo a nonempty subset $\Delta$.

Another related theorem of Kwack [1] states:

THEOREM 3.11. Let $X$ be a complex manifold and A a complex subspace of codimension at least 2. Let $Y$ be a complete hyperbolic space. Then every holomorphic map $f: X-A \rightarrow Y$ extends to a holomorphic map $f: X \rightarrow Y$.

For a slightly more general result, see Kobayashi [4].

We shall discuss later holomorphic and meromorphic extension problems 
more systematically. We have the following analogue of Theorem 3.4. For the sake of simplicity, we consider the case where $\Delta$ is empty.

THEOREM 3.12. Let $Y$ be hyperbolically imbedded in $Z$. Let $\tilde{Z}$ be a complex space with a proper holomorphic map $\pi: \tilde{Z} \rightarrow z$ such that $\pi^{-1}(z)$ is discrete (and hence finite) for each $z \in Z$. (In particular, let $\tilde{Z}$ be the normalization of $Z$.) Let $\tilde{Y}=\pi^{-1}(Y)$. Then $\tilde{Y}$ is hyperbolically imbedded in $\tilde{Z}$.

In examining examples later we shall find that when $Y$ is hyperbolically imbedded in $Z, Y$ is often complete hyperbolic. This is based on the following fact (see Kiernan and Kobayashi [2]).

THEOREM 3.13. Let $Y$ be hyperbolically imbedded in $Z$ modulo a subset $\Delta$. Assume that $Y$ is locally complete in the sense that every point $p$ of $\partial Y(=\bar{Y}-Y)$ has a neighborhood $V$ in $Z$ such that $V \cap Y$ is complete hyperbolic. (This assumption is satisfied if every $p \in \partial Y$ has a neighborhood $V$ such that $\mathrm{V}-\mathrm{Y}$ is the zero set of a single holomorphic function in $\mathrm{V}$.) Then $\mathrm{Y}$ is complete hyperbolic modulo $\Delta \cap Y$.

So far we have discussed spaces $X$ for which $d_{X}$ is a distance. We want to comment briefly on spaces $X$ for which $c_{X}$ is a distance. Let $\pi: \tilde{X} \rightarrow X$ be a covering projection. In contrast to the case of $d_{X}$ (see Theorem 3.3), $c_{X}$ can be trivial even when $c_{\tilde{X}}$ is a distance. For example, let $X$ be a compact Riemann surface of genus $\geqq 1$ and $\tilde{X}$ the unit disk. For this reason we introduce the concept of a Carathéodory hyperbolic (or C-hyperbolic for short) space. A complex space $X$ is said to be $C$-hyperbolic if it has a covering space $\tilde{X}$ such that $c_{\tilde{X}}$ is locally nondegenerate in the sense that each point $p \in \tilde{X}$ has a neighborhood $V$ such that $c_{\tilde{X}}(p, q)>0$ for $q \in V, q \neq p$. Then the induced inner pseudo-distance $c_{\tilde{X}}^{i}$ is a distance. Since $d_{\tilde{X}} \geqq c_{\tilde{X}}^{i}, \tilde{X}$ is hyperbolic and, by Theorem 3.3, $X$ itself is hyperbolic. (This definition is slightly more general than the one in my monograph where it is required that $c_{\tilde{X}}$ be a distance.) We say that a $C$-hyperbolic space $X$ is complete if $\tilde{X}$ is Cauchy complete with respect to $c_{\tilde{X}}^{i}$. From $d_{\tilde{X}} \geqq c_{\tilde{X}}^{i}$ and Theorem 3.3 it follows that every complete $C$-hyperbolic space is complete hyperbolic.

4. Metric and holomorphic completeness. In this section we consider only bounded domains in $\boldsymbol{C}^{n}$ although some of the results are valid for more general complex spaces.

A metric space is said to be finitely compact if every bounded closed set is compact. We do not know if the Cauchy completeness of a bounded domain $X$ with respect to $c_{X}$ implies the finite compactness with respect to $c_{X}$ (in contrast to the distance $\left.d_{X}\right)$. We denote by $H^{\infty}(X)$ the algebra of bounded holomorphic functions on $X$. A domain $X$ is said to be $H^{\infty}$-convex if it is convex with respect to $H^{\infty}(X)$. I do not define here the concept of "generalized analytic polyhedron" (see Kobayashi [4], [6]); it suffices to say that it encompasses balls in $\boldsymbol{C}^{n}$ as well as the analytic polyhedra. Now we state

THEOREM 4.1. For a bounded domain $X$ in $\boldsymbol{C}^{n}$, we have the following 
diagram of implications:

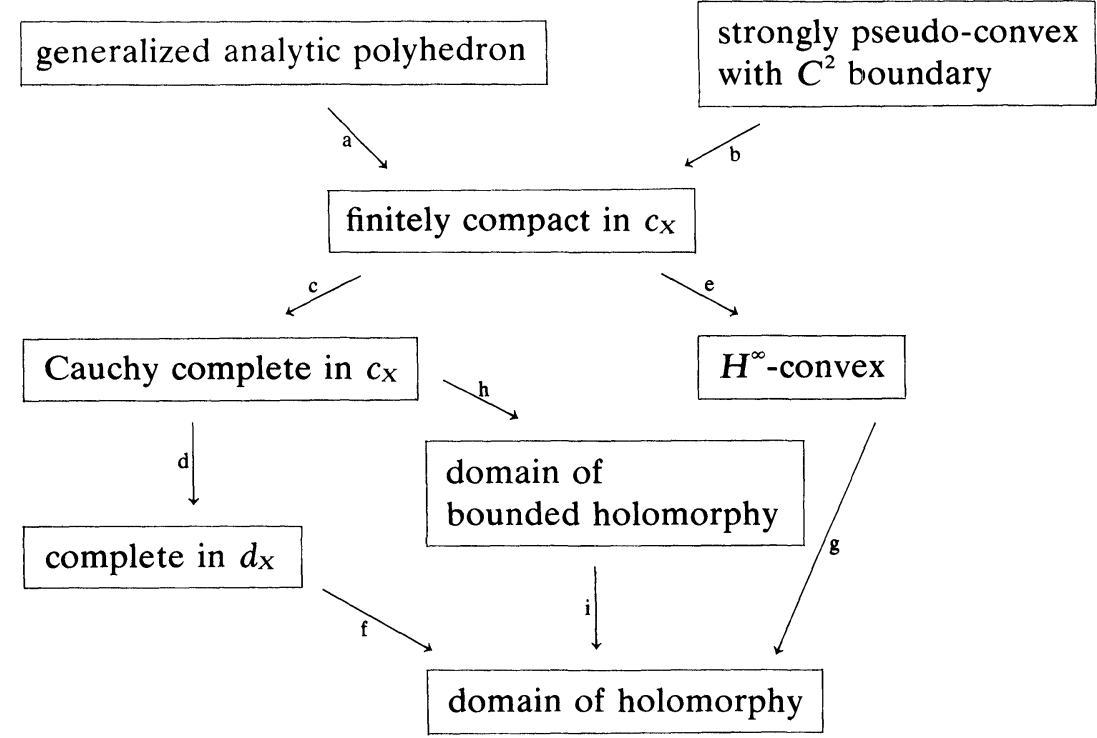

Implication $b$ is due to Graham [1]. For the remaining implications, see Kobayashi [4] and Sibony [1], [2]. In the 1-dimensional case, $X$ is $H^{\infty}$ convex if and only if it is a domain of bounded holomorphy, but no implication in either direction in higher dimensions, see Sibony [1] and Ahern and Schneider [1]. The punctured disk $D^{*}$ provides an example showing that $\left(d^{-1}\right)$ is not valid. For counterexamples to the implication $\left(e^{-1}\right)$, see Ahern and Schneider [1] as well as Sibony [1], [2]. I do not know if $\left(c^{-1}\right)$ is valid or not.

With respect to the Bergman metric $d s_{X}^{2}$, we have

TheORem 4.2. For a bounded domain $X$ in $\mathbf{C}^{n}$, we have the following diagram of implications:

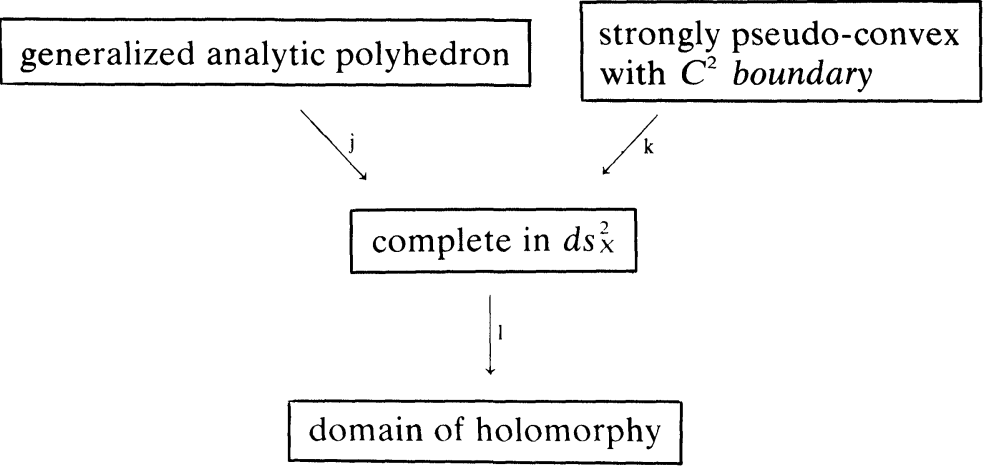

For the implication k, see $\mathrm{Wu}$ and Greene $[\mathbf{1}]$ and Kerzman [1]. For $\mathrm{j}$ and 1, see Bremermann [1] and Kobayashi [6]. Again, the punctured disk $D^{*}$ serves as a counterexample to $\left(l^{-1}\right)$. 
The study of the boundary behavior of $c_{X}, d_{X}$ and $d s_{X}^{2}$ is clearly relevant to the question of completeness with respect to these metrics. For the boundary behavior of $c_{\mathrm{X}}$ and $d_{\mathrm{X}}$ on pseudo-convex domains, see Graham [1]. For the boundary behavior of the Bergman metric $d s_{X}^{2}$, see Diederich [1].

5. Intrinsic volume forms. Let $X$ be a complex space. Then we can construct measures on $X$ using the pseudo-distances $c_{X}$ and $d_{X}$. In general, given a topological space $X$ with a pseudo-distance $d$ and a nonnegative real number $r$, we can define the so-called $r$-dimensional Hausdorff measure.

On the other hand, for each positive integer $k \leqq n(=\operatorname{dim} X)$, we can construct two types of $2 k$-dimensional measures on $X$ without using $c_{X}$ or $d_{X}$ but imitating the construction of $c_{X}$ and $d_{X}$. These measures have been studied by Eisenman [1] and Kobayashi [4], but they have proved interesting so far only in the top dimension. We shall describe them briefly in dimension $2 n$ as differential forms of degree $2 n$ rather than Borel measures as in the above mentioned monographs.

Let $X$ be a complex space of dimension $n$. Let $D^{n}$ be the unit polydisk in $\boldsymbol{C}^{n}$ with the invariant volume form $\mu$ defined by

$$
\mu=\prod_{j=1}^{n} \frac{4 \sqrt{ }-1}{\left(1-\left|z^{j}\right|^{2}\right)^{2}} d z^{j} \wedge d \bar{z}^{j}
$$

with respect to the natural coordinate system $z^{1}, \cdots, z^{n}$ of $\boldsymbol{C}^{n}$. We define an intrinsic pseudo-volume form $\Phi_{X}$ generalizing $\mu$ and analogous to the Carathéodory pseudo-metric $E_{X}$ by setting

$$
\left(\Phi_{X}\right)_{x}=\sup _{f}\left(f^{*} \mu\right)_{x}
$$

where the supremum is taken over all holomorphic maps $f: X \rightarrow D^{n}$. It is safer to consider $\Phi_{X}$ as a form defined only at the nonsingular points of $X$ since the tangent space at a singular point has a higher dimension than $n$.

To obtain an intrinsic pseudo-volume form $\Psi_{X}$ analogous to $F_{X}$, we set

$$
\left(\Psi_{X}\right)_{x}=\inf _{f}\left(f^{-1}\right)^{*}(\mu)_{0},
$$

where the infimum is taken over all holomorphic maps $f: D^{n} \rightarrow X$ which send the origin $0 \in D^{n}$ to $x \in X$ and are nondegenerate at 0 . Again, $\Psi_{X}$ is defined only at the regular points of $X$. By allowing $f$ to be meromorphic (but holomorphic and nondegenerate at 0 ) in the definition above, we obtain another intrinsic pseudo-volume form $\tilde{\Psi}_{x}$, which will be useful in birational geometry (see Yau [2]). (By allowing $f$ to be meromorphic in the definition of $\Phi_{X}$, we obtain nothing new since every meromorphic map $f: X \rightarrow D^{n}$ is holomorphic.)

The basic properties of these pseudo-volume forms may be summarized by the following theorem. (The proofs of these assertions are similar to those of the corresponding statements for $E_{X}$ and $F_{X}$.) 
TheOREM 5.1. Let $X$ and $Y$ be complex spaces of dimension $n$.

$$
\mu=\Phi_{D^{n}}=\Psi_{D^{n}}=\tilde{\Psi}_{D^{n}}
$$

(2) If $f: X \rightarrow Y$ is holomorphic, then

$$
f^{*} \Phi_{\mathrm{Y}} \leqq \Phi_{\mathrm{X}} \text { and } f^{*} \Psi_{\mathrm{Y}} \leqq \Psi_{\mathrm{X}}
$$

(3) If $f: X \rightarrow Y$ is meromorphic, then

$$
f^{*} \Phi_{Y} \leqq \Phi_{X} \text { and } f^{*} \tilde{\Psi}_{Y} \leqq \tilde{\Psi}_{X} ;
$$

(4) If $\Omega_{X}$ is any pseudo-volume form of $X$ such that $f^{*} \Omega_{X} \leqq \mu$ for all holomorphic (resp. meromorphic) maps $f: D^{n} \rightarrow X$, then

$$
\Omega_{\mathrm{X}} \leqq \Psi_{\mathrm{X}} \quad\left(\operatorname{resp} . \Omega_{\mathrm{X}} \leqq \tilde{\Psi}_{\mathrm{X}}\right)
$$

(5) If $\Omega_{X}$ is any pseudo-volume form on $X$ such that $f^{*} \mu \leqq \Omega_{X}$ for all holomorphic maps $f: X \rightarrow D^{n}$, then

$$
\begin{gathered}
\Phi_{X} \leqq \Omega_{X} ; \\
\Phi_{X} \leqq \tilde{\Psi}_{X} \leqq \Psi_{X} ;
\end{gathered}
$$

(7) If $\tilde{X}$ is a covering space of $X$ with projection $\pi: \tilde{X} \rightarrow X$, then

$$
\Psi_{\tilde{\mathrm{X}}}=\pi^{*} \Psi_{\mathrm{X}} \text { and } \tilde{\Psi}_{\mathrm{X}}=\pi^{*} \tilde{\Psi}_{\tilde{\mathrm{X}}}
$$

(8) $\Psi_{X}$ and $\tilde{\Psi}_{X}$ are upper semicontinuous and $\Phi_{X}$ is continuous.

The Bergman kernel form $B_{X}$ given by (2.9) is defined and smooth at the regular points of $X$. We have a weaker version of Theorem 5.1(2):

$$
B_{X^{\prime}} \geqq B_{X} \text { on } X^{\prime} \text { if } X^{\prime} \text { is a domain in } X \text {. }
$$

Corresponding to Theorem 2.4, we have

Theorem 5.2. If $X$ is normal and $A=X-X^{\prime}$ is contained in an analytic subset of codimension at least 1 in $X$, then

$$
\Phi_{\mathrm{X}^{\prime}}=\Phi_{\mathrm{X}} \text { and } B_{\mathrm{X}^{\prime}}=\mathrm{B}_{\mathrm{X}} \text { on } \mathrm{X}^{\prime} \text {. }
$$

But we cannot expect anything like (2) of Theorem 2.4 for $\Psi_{X}$.

We say that a complex space $X$ is measure-hyperbolic (resp. meromorphically measure-hyperbolic) if $\Psi_{X}$ (resp. $\tilde{\Psi}_{X}$ ) is positive outside an analytic subset of codimension at least 1 . Then

THEOREM 5.3. (1) Let $\pi: \tilde{X} \rightarrow X$ be a holomorphic map such that $\pi^{-1}(x)$ is discrete for each $x \in X$. If $X$ is (meromorphically) measure-hyperbolic, so is $\tilde{X}$

(2) If $\pi$ is an unbranched covering projection, $\tilde{X}$ is (meromorphically) measure-hyperbolic if and only if $X$ is.

Although I do not know a precise relation between $\Psi_{X \times Y}$ and $\Psi_{X} \wedge \Psi_{Y}$, we have

THEOREM 5.4. If $X$ and $Y$ are (meromorphically) measure-hyperbolic, so is $\mathrm{X} \times \mathrm{Y}$. 
Applying (4) of Theorem 5.1 to the $2 n$-dimensional Hausdorff measure defined by $d_{x}$, we obtain

THEOREM 5.5. If a complex space $X$ is hyperbolic modulo an analytic subset of codimension at least 1 , it is measure-hyperbolic.

The principle of the equidimensional little Picard theorem is given by

THEOREM 5.6. Let $X$ be a complex space such that $\Psi_{X}=0$ (resp. $\left.\tilde{\Psi}_{X}=0\right)$ on a nonempty open subset. Let $\mathrm{Y}$ be a measure-hyperbolic (resp. meromorphically measure-hyperbolic) complex space of the same dimension. Then every holomorphic (resp. meromorphic) map $f: X \rightarrow Y$ is everywhere degenerate.

It is easy to see that $\Psi_{X}=0$ if (i) $X=C \times X^{\prime}$, (ii) $X=T \times X^{\prime}$, where $T$ is a complex torus, (iii) $X=P_{k}(C) \times X^{\prime}$. More generally, if $X$ is a complex space on which a complex Lie group acts, then $\Psi_{X}=0$; (this observation is due to a discussion with Bun Wong). To see this, take any regular point $x$ of $X$ which is not fixed by the group (which may be assumed to be the 1-parameter group $\boldsymbol{C}$ ). Then imbed $D^{n-1}$ in $X$ so that the imbedded $D^{n-1}$ is transversal to the orbit of $\boldsymbol{C}$ through $x$. Translating the imbedded $D^{n-1}$ along the orbit by the group action of $\boldsymbol{C}$, we obtain a holomorphic map $f: C \times D^{n-1} \rightarrow X$ which is nondegenerate at $x$. Since $f$ is volume-decreasing, $\Psi_{X}$ vanishes at $x$.

6. Schwarz lemma and differential geometric criteria for hyperbolicity. Given a complex manifold $X$, it is often a difficult problem to determine whether or not $X$ is hyperbolic. In this section we want to give differential geometric criteria for hyperbolicity and measure hyperbolicity. They are based on generalizations of the Schwarz lemma.

As in $\$ 2$, we denote the Poincaré metric of curvature -1 on the unit disk $D$ by $d s^{2}$; see (2.1). The following generalization by Ahlfors [1] reveals the differential geometric nature of the Schwarz-Pick lemma.

THEOREM 6.1. Let $d \sigma^{2}$ be any hermitian pseudo-metric on $D$ whose curvature is bounded above by -1 . Then $d \sigma^{2} \leqq d s^{2}$.

REMARK. The term "pseudo-metric" means that $d \sigma^{2}$ is only positive semidefinite. The assumption is that the curvature be bounded by -1 wherever $d \sigma^{2}$ is positive. The theorem holds even when $d \sigma^{2}$ is only continuous at zero points of $d \sigma^{2}$ as long as it is twice differentiable at the points where it is positive. This fact is useful in applications. In fact, the theorem holds for an upper semicontinuous $d \sigma^{2}$ with "supporting pseudo-metrics", for details, see Ahlors [1], [2].

As a consequence of Theorem 6.1, we obtain

THEOREM 6.2. Let $X$ be a complex manifold with a hermitian pseudometric $d s_{X}^{2}$. If its holomorphic sectional curvature is bounded above by -1 , then every holomorphic map $f: D \rightarrow X$ is distance-decreasing, i.e., $f^{*} d s_{X}^{2} \leqq d s^{2}$ on $D$.

To prove this, one has only to apply Theorem 6.1 to $d \sigma^{2}=f^{*} d s_{X}^{2}$. The 
equation of Gauss-Codazzi for hermitian submanifolds implies that the curvature of $d \sigma^{2}$ does not exceed that of $d s_{X}^{2}$ and makes Theorem 6.1 applicable to $d \sigma^{2}=f^{*} d s_{X}^{2}$ (see Kobayashi [2], [4]).

If the holomorphic sectional curvature of $d s_{X}^{2}$ is bounded by a negative constant $-A$, we shall use the expression "negatively bounded curvature". In this case, $A d s_{X}^{2}$ has holomorphic sectional curvature $\leqq-1$.

Examining the proof of Theorem 6.2, we see that the assumption on the holomorphic sectional curvature was made to insure that $f^{*} d s_{X}^{2}$ has curvature bounded above by -1 . It is possible to extend Theorem 6.2 to a more general metric. Let $G_{X}$ be a different pseudo-metric as in (2.27) and use the definition of the curvature $k_{X}$ defined by (2.30). Then (Grauert and Reckziegel [1])

THEOREM 6.3. Let $X$ be a complex manifold with a differential pseudometric $G_{X}$ which is twice differentiable outside the zero section of $T(X)$. If its holomorphic sectional curvature $k_{X}$ is bounded above by -1 , then every holomorphic map $f: D \rightarrow X$ is distance-decreasing.

Let $G_{X}$ be a differential pseudo-metric as above and let $\Delta$ be the set of points $x \in X$ where $G_{X}$ is degenerate, i.e., $G_{X}(v)=0$ for some nonzero vector $v \in T_{x}(X)$. Let $\delta_{X}$ be the distance function defined by $G_{X}$; it is a pseudodistance in general. By (2) of Theorem 2.2 and Theorem 6.3, we have

$$
\delta_{\mathrm{X}} \leqq d_{\mathrm{X}}
$$

Hence, we have the following criterion for hyperbolicity.

THEOREM 6.4. Let $X$ be a complex manifold with a differential pseudometric $G_{X}$ which is twice differentiable outside the zero section of $T(X)$. If its holomorphic sectional curvature is negatively bounded, then $X$ is hyperbolic modulo the set $\Delta$ of points where $G_{X}$ is degenerate. If $G_{X}$ is complete modulo $\Delta$ (i.e., $\delta_{\mathrm{X}}$ is complete modulo $\Delta$ ), then $X$ is complete hyperbolic modulo $\Delta$.

In order to generalize Theorem 6.1 to the case of higher dimension, we consider a volume form and the associated Ricci form. If $X$ is a complex manifold of dimension $n$ with local coordinate system $z^{1}, \cdots, z^{n}$, then a pseudo-volume form $v_{X}$ on $X$ can be locally written as

$$
v_{\mathrm{X}}=V \cdot \prod_{j=1}^{n}\left(\sqrt{ }-1 d z^{j} \wedge d \bar{z}^{j}\right),
$$

where $V$ is a nonnegative function. If $V$ is positive everywhere, $v_{x}$ is a volume form of $X$. To each $v_{X}$ given by $(6.2)$, we associate a real $(1,1)$-form Ric $v_{M}$, called the associated Ricci form, as follows.

$$
\text { Ric } v_{\mathrm{X}}=-d d^{\mathrm{c}} \log V=2(-1)^{1 / 2} \sum R_{\mathrm{j} \overline{\mathrm{k}}} d z^{j} \wedge d \bar{z}^{k},
$$

where

$$
R_{j \bar{k}}=-\partial^{2} \log V / \partial z^{j} \partial \bar{z}^{k}
$$

The Ricci form Ric $v_{\mathrm{X}}$ is defined only at the points where $v_{\mathrm{X}}$ is strictly 
positive. If $v_{X}$ is strictly positive everywhere, then the closed 2-form $(4 \pi)^{-1}$ Ric $v_{X}$ defines the first Chern class $c_{1}(X)$ of $X$. If $X$ is a hermitian manifold with hermitian 2-form $\varphi_{X}$ and volume form $v_{X}=\varphi_{X}^{n}$, then $\left(R_{j \bar{k}}\right)$ is usually called the Ricci tensor. Hence the name "Ricci form" for Ric $v_{X}$. If $\operatorname{dim} X=1$, then

$$
\text { Ric } v_{\mathrm{X}}=K_{\mathrm{X}} v_{\mathrm{X}}
$$

where $K_{X}$ is the (Gaussian) curvature of $X$. We say that Ric $v_{X}$ is negative if the matrix $\left(R_{j \bar{k}}\right)$ is negative definite wherever defined. We say that it is negatively bounded if it is negative and if

$$
K_{X}:=-\left(-\operatorname{Ric} v_{X}\right)^{n} / v_{X} \leqq-c<0
$$

wherever defined.

Let $D^{n}$ be the unit polydisk in $\boldsymbol{C}^{n}$ and $\mu$ the volume form on $D^{n}$ defined by (5.1). Then

$$
\operatorname{Ric} \mu=-4(-1)^{1 / 2} \sum_{j=1}^{n} \frac{1}{\left(1-\left|z^{j}\right|^{2}\right)^{2}} d z^{j} \wedge d \bar{z}^{j}
$$

and

$$
K:=-(-\operatorname{Ric} \mu)^{n} / \mu=-1 .
$$

Theorem 6.1 can be generalized to $D^{n}$ in the following manner:

THEOREM 6.5. Let $v_{D^{n}}$ be any pseudo-volume form on $D^{n}$ with negatively bounded Ric $v_{D^{n}}$ and normalized in such a way that $K_{D^{n}}:=-\left(-\operatorname{Ric} v_{D^{n}}\right)^{n} / v_{D^{n}} \leqq-1$. Then $v_{D^{n}} \leqq \mu$.

The remark following Theorem 6.1 about differentiability applies also to Theorem 6.5. As a consequence of Theorem 6.5, we have the following equidimensional analogue of Theorem 6.2.

THEOREM 6.6. Let $X$ be an $n$-dimensional complex manifold with pseudovolume form $v_{\mathrm{X}}$ such that Ric $v_{\mathrm{X}}$ is negatively bounded and $K_{X}=-\left(-\operatorname{Ric} v_{X}\right)^{n} / v_{X} \leqq-1$. Then every meromorphic map $f: D^{n} \rightarrow X$ is volume-decreasing in the sense that $f^{*} v_{X} \leqq \mu$.

If $f$ is holomorphic, the theorem is immediate. If $f$ is meromorphic, let $S$ be the indeterminacy set for $f$. Since Ric $v_{x}$ is negative, the coefficient $V$ of $v_{\mathrm{X}}$ is plurisubharmonic. Hence the coefficient of $f^{*} v_{\mathrm{X}}$ is a plurisubharmonic function on $D^{n}-S$. Since $S$ has codimension at least $2, f^{*} v_{\mathrm{x}}$ extends across $S$ (see Grauert and Remmert [1]), and Theorem 6.5 applies to $f^{*} v_{X}$.

From Theorems 5.1 and 6.6 we conclude

THEOREM 6.7. Let $X$ and $v_{X}$ be as in Theorem 6.6. Then $v_{X} \leqq \tilde{\Psi}_{X} \leqq \Psi_{X}$, where $\Psi_{\mathrm{X}}$ and $\tilde{\Psi}_{\mathrm{X}}$ are the intrinsic pseudo-volume forms defined in $\$ 5$. In particular, if $v_{\mathrm{X}}$ is positive outside an analytic subset of codimension at least 1 , then $X$ is measure-hyperbolic (in fact, even meromorphically measurehyperbolic).

The criterion for hyperbolicity given by Theorem 6.4 is not very easy to 
use; it is usually a major task to construct a hermitian or differential metric and compute its curvature. On the other hand, Theorem 6.7 is much easier to use partly because Ric $v_{X}$ can be calculated easily and partly because the explicit calculation of Ric $v_{x}$ can be avoided as we shall explain in the next section.

In the preceding section we alluded to intermediate dimensional measures. But there is no theorem similar to Theorems 6.2 and 6.6 for these measures for lack of a suitable concept of "intermediate dimensional curvature", i.e., a concept between holomorphic sectional curvature and Ricci curvature. Although I used the word "between", I did not mean that the holomorphic sectional curvature is a richer concept than the Ricci curvature. Nevertheless it seems likely that "negative holmorphic sectional curvature" implies "negative Ricci curvature"; Theorem 5.5 is certainly a supporting evidence.

The generalized Schwarz lemma, Theorem 6.2, holds for domains a little more general than the unit disk; see Kobayashi [4], Yau [3]. However Theorems 6.2 and 6.3 suffice for most applications since a mapping defined on a general complex space can be always restricted to a small disk. This is true also with Theorem 6.6.

The equidimensional Schwarz lemma in higher dimension was first obtained by Dinghas [1] for maps into Einstein-Kähler manifolds of negative Ricci curvature and by Chern [3] for maps into Einstein-Hermitian manifolds of negative Ricci curvature. Theorem 6.6 in its general form is due to Kobayashi [1]. As we shall see in the next section, it is essential for algebraic geometric applications to be rid of Kähler or hermitian metrics and to consider pseudo-volume forms directly as in Theorem 6.6.

An interesting application of the Schwarz-Ahlfors lemma, Theorem 6.1, is to the problem of determining the Bloch constant; see Ahlfors [2] and references therein and Chern [3].

Attempts have been made to generalize the Schwarz lemma to harmonic maps between Riemannian manifolds; see Kiernan [7] and Chern and Goldberg [1].

7. Ample canonical bundles and manifolds of general type. In the preceding section, we stated the equidimensional Schwarz lemma for maps into a complex manifold carrying a pseudo-volume form with negatively bounded Ricci form. In this section, we shall give algebraic geometric criteria for the existence of such a pseudo-volume form.

Let $X$ be a compact complex manifold. A complex line bundle $L$ is said to be very ample if it has enough holomorphic sections to induce an imbedding of $X$ into a projective space in the following precise sense. Let $\Gamma(L)$ be the space of holomorphic sections of $L$ and $N+1=\operatorname{dim} \Gamma(L)$. For each $x \in X$, let $\Gamma(L)_{x}$ be the subspace consisting of sections vanishing at $x$. Assume that, for each $x, \Gamma(L)_{x} \neq \Gamma(L)$ so that $\operatorname{dim} \Gamma(L)_{x}=N$. Then we obtain a map $j: X \rightarrow$ $P^{*}(\Gamma(L))$, where $\left.P^{*}(\Gamma)\right)$ is the projective space of hyperplanes of $\Gamma(L)$. (We used the notation $P^{*}$ to indicate that it is the dual projective space over 
$\Gamma(L)$.) The map $j$ sends $x$ to $\Gamma(L)_{x}$. For our purpose it is convenient to fix a basis $\sigma_{0}, \sigma_{1}, \cdots, \sigma_{N}$ of $\Gamma(L)$ and to define $j$ as a map sending $x$ to $\left(\sigma_{0}(x), \cdots, \sigma_{\mathrm{N}}(x)\right) \in P_{\mathrm{N}}(C)$. If $j: X \rightarrow P_{\mathrm{N}}(C)$ is an imbedding, $L$ is said to be very ample. A line bundle $L$ is said to be ample if there exists a positive integer $k$ such that $L^{k}$ is very ample.

Let $K$ be the canonical line bundle of $X$, i.e., $K=\Lambda^{n} T^{*}(X)$, so that the holomorphic sections of $K$ are the holomorphic $n$-forms on $X$ (where $n=\operatorname{dim} X)$. A holomorphic section $\sigma$ of $K^{k}$ can be expressed locally as

$$
\sigma=f(z)\left(d z^{1} \wedge \cdots \wedge d z^{n}\right)^{k},
$$

where $z^{1}, \cdots, z^{n}$ is a local coordinate system of $X$ and $f$ is a function holomorphic in the coordinate neighborhood. Let $\sigma_{0}, \cdots, \sigma_{\mathrm{N}}$ be a basis of $\Gamma\left(K^{k}\right)$ and let

$$
\sigma_{i}=f_{i}(z)\left(d z^{1} \wedge \cdots \wedge d z^{n}\right)^{k} .
$$

Define a pseudo-volume form $v_{\mathrm{X}}$ by setting

$$
v_{\mathrm{X}}=\left(\sum_{i=0}^{N}\left|f_{i}\right|^{2}\right)^{1 / k}(\sqrt{ }-1)^{n^{2}} d z^{1} \wedge \cdots \wedge d z^{n} \wedge d \bar{z}^{1} \wedge \cdots \wedge d \bar{z}^{n} .
$$

Symbolically we may write

$$
v_{X}=\left(\sum_{i=0}^{N}\left|\sigma_{i} \wedge \bar{\sigma}_{i}\right|\right)^{1 / k}
$$

Assume that $K^{k}$ is very ample. Then $v_{X}$ is a volume form and its associated Ricci form Ric $v_{X}$ is negative. In fact, if we pull back the Fubini-Study metric of $P_{N}(\boldsymbol{C})$ by $j: X \rightarrow P_{N}(\boldsymbol{C})$, then its Kähler form differs only by the sign from Ric $v_{\mathrm{X}}$.

Conversely, if a compact complex manifold $X$ admits a volume form $v_{X}$ with negative Ric $v_{X}$, then its canonical line bundle $K$ is ample; this is a consequence of the result of Kodaira [1].

It is not known whether a compact complex manifold $X$ with ample canonical line bundle $K$ admits a Kähler metric of negative Ricci tensor; this is part of the problem posed by Calabi [1]. As I have already pointed out, it is therefore important in Theorem 6.6 not to restrict ourselves to the volume form derived from a Kähler metric.

Theorems 6.6 and 6.7 are stated for pseudo-volume forms. Pseudovolume forms arise in a rather natural manner. Let $X$ and $\tilde{X}$ be $n$ dimensional complex manifolds and $\pi: \tilde{X} \rightarrow X$ a holomorphic map whose differential $\pi_{*}: T(\tilde{X}) \rightarrow T(X)$ is nondegenerate outside an analytic subset $\Delta$ of $\tilde{X}$. If $v_{X}$ is a volume form on $X$, then $\pi^{*} v_{X}$ is a pseudo-volume form on $\tilde{X}$ which is positive outside $\Delta$. If Ric $v_{X}$ is negatively bounded, so is Ric $\pi^{*} v_{X}$ $\left(=f^{*}\left(\operatorname{Ric} v_{X}\right)\right)$ outside $\Delta$. For example, if we blow up a compact complex manifold $X$ with ample canonical line bundle at a point or, more generally, along a subvariety $\Delta$, then we obtain a manifold $\tilde{X}$ admitting a pseudovolume form with negatively bounded Ricci form. The manifold $\tilde{X}$ thus obtained is an example of an algebraic manifold of general type. 
An $n$-dimensional compact complex manifold $X$ is said to be of general type if its canonical line bundle $K$ satisfies

$$
\sup \lim _{m \rightarrow \infty} \frac{1}{m^{n}} \operatorname{dim} \Gamma\left(K^{m}\right)>0 .
$$

This definition says that the so-called Kodaira dimension of $X$ is equal to the complex dimension of $X$ (see Iitaka [1]). Assume further that $X$ is projective algebraic and let $L$ be a very ample line bundle over $X$. Then (see Kodaira [3] and Kobayashi and Ochiai [3]) there exists a positive integer $m$ such that

$$
\Gamma\left(K^{m} L^{-1}\right) \neq 0 .
$$

Fixing a nontrivial element $\alpha$ of $\Gamma\left(K^{m} L^{-1}\right)$, we define an injection

$$
\varphi \in \Gamma(L) \rightarrow \alpha \varphi \in \Gamma\left(K^{m}\right) .
$$

Let $\varphi_{0}, \varphi_{1}, \cdots, \varphi_{N}, N+1=\operatorname{dim} \Gamma(L)$, be a basis for $\Gamma(L)$. Then $\alpha \bar{\alpha} \sum_{i=0}^{N} \varphi_{i} \bar{\varphi}_{i}$ may be considered as a $C^{\infty}$ section of $\left(K^{m} L^{-1}\right) L \otimes\left(\bar{K}^{m} \bar{L}^{-1}\right) \bar{L}=K^{m} \otimes \bar{K}^{m}$ and can be locally expressed as

$$
|a(z)|^{2} \sum_{i=0}^{N}\left|h_{i}(z)\right|^{2}\left(\prod_{k=1}^{n} \sqrt{ }-1 d z^{k} \wedge d \bar{z}^{k}\right)^{m}
$$

where $a(z), h_{0}(z), \cdots, h_{N}(z)$ are locally defined holomorphic functions corresponding to the sections $\alpha, \varphi_{0}, \cdots, \varphi_{N}$. Then the pseudo-volume form $v_{X}$ defined by

$$
v_{\mathrm{X}}=\left(|a(z)|^{2} \sum_{i=0}^{N}\left|h_{i}(z)\right|^{2}\right)^{1 / m}\left(\prod_{k=1}^{n} \sqrt{ }-1 d z^{k} \wedge d \bar{z}^{k}\right)
$$

vanishes exactly where the section $\alpha$ vanishes, and its Ricci form Ric $v_{\mathrm{X}}$ is negatively bounded (outside the zeroes of $\alpha$ ).

We can summarize the results of this section so far by the following

THEOREM 7.1. For a compact complex manifold $X$, consider the following conditions:

(1) Its canonical line bundle $K$ is ample.

(2) It admits a volume form $v_{\mathrm{X}}$ with negatively bounded Ric $v_{\mathrm{X}}$.

(3) $\tilde{\Psi}_{X}$ is positive everywhere.

(4) $\Psi_{X}$ is positive everywhere.

(1') It is a compact complex manifold of general type.

(2') It admits a pseudo-volume form $v_{\mathrm{X}}$ which is positive outside an analytic subset $\Delta$ and has negatively bounded Ric $v_{\mathrm{x}}$ (outside $\Delta$ ).

(3') $\tilde{\Psi}_{X}$ is positive outside an analytic subset $\Delta$, i.e., $X$ is meromorphically measure-hyperbolic.

(4') $\Psi_{X}$ is positive outside an analytic subset $\Delta$, i.e., $X$ is measurehyperbolic.

Then we have the following implications between these conditions:

$$
\begin{array}{cccc}
(1) & \leftrightarrow(2) & \rightarrow(3) & \rightarrow(4) \\
\downarrow & \downarrow & \downarrow & \downarrow \\
\left(1^{\prime}\right) & \rightarrow\left(2^{\prime}\right) & \rightarrow\left(3^{\prime}\right) & \rightarrow\left(4^{\prime}\right) .
\end{array}
$$


The vertical implications are all sharp, but it is not known whether any of the horizontal implications is an equivalence (except $(1) \leftrightarrow(2)$ ). The argument used in the proof of Theorem 6.6, i.e., extension of plurisubharmonic functions, shows that condition $\left(2^{\prime}\right)$ is a bimeromorphic invariant. Both ( $\left.1^{\prime}\right)$ and $\left(3^{\prime}\right)$ are bimeromorphically invariant, but it is not clear if $\left(4^{\prime}\right)$ is so. In deriving the implication $\left(1^{\prime}\right) \rightarrow\left(2^{\prime}\right)$, we use the fact that a manifold of general type is a Moišezon space and hence is bimeromorphic to a projective algebraic manifold (Moišezon [1]). (We recall that a Moišezon space is a compact complex space whose function fields has transcendence degree equal to the dimension of the space.)

From Theorem 5.6 and the implication $\left(1^{\prime}\right) \rightarrow\left(3^{\prime}\right)$, we obtain

THEOREM 7.2. Let $X$ be an $n$-dimensional complex space bimeromorphic to a space on which a complex Lie group of positive dimension is acting. Let $Y$ be an $n$-dimensional Moišezon space of general type. Then every meromorphic map $f: X \rightarrow Y$ is everywhere degenerate.

Iitaka [2] made the following conjecture:

If $X$ is a projective algebraic manifold whose universal covering space is $\boldsymbol{C}^{n}$, then it admits an abelian variety as a finite unramified covering space.

This is known to be true for $n=2$ and Iitaka obtained partial results in dimension $\geqq 3$. He used the special case $X=\boldsymbol{C}^{n}$ of Theorem 7.2 to show that the Kodaira dimension of such a manifold is less than $n$.

While we have a reasonably simple algebraic sufficient condition for measure-hyperbolicity, we have only the following condition for hyperbolicity which is probably too strong to be useful.

THEOREM 7.3. Let $X$ be a compact complex manifold with ample cotangent bundle. Then $X$ admits a complex Finsler metric with negative holomorphic bisectional curvature and, hence, is hyperbolic.

I am using the term "ample" here in the sense most algebraic geometers use. For the proof, see Kobayashi [7].

8. Families of holomorphic and meromorphic maps. According to Douady [1], the family $H(z)$ of compact complex subspaces of a complex space $Z$ is, in a natural manner, a complex space (with many components). This is classical when $Z$ is projective algebraic-Chow coordinates and Chow varieties.

Given two complex spaces $X$ and $Y$, let $\operatorname{Hol}(X, Y)(\operatorname{resp} . \operatorname{Mer}(X, Y))$ denote the family of holomorphic (resp. meromorphic) maps $f: X \rightarrow Y$. Since the graph of $f \in \operatorname{Mer}(X, Y)$ is a complex subspace of $X \times Y$, we may consider both $\operatorname{Hol}(X, Y)$ and $\operatorname{Mer}(X, Y)$ as complex subspaces of $H(X \times Y)$ provided that $X$ is compact. If $X$ is noncompact, $\operatorname{Hol}(X, Y)$ can be a large set of infinite dimension as in the case of $X=Y=\boldsymbol{C}^{n}$.

In this section we are interested in showing that $\operatorname{Hol}(X, Y)$ and $\operatorname{Mer}(X, Y)$ cannot be too large if $Y$ is hyperbolic or measure-hyperbolic. At the outset, it should be pointed out that $\operatorname{Hol}(X, Y)=\operatorname{Mer}(X, Y)$ if $Y$ is compact hyperbolic. This will be proved in $\S 10$. 
For the following theorem, see Kobayashi [4].

THEOREM 8.1. Let $X$ and $Y$ be complex spaces. If $Y$ is complete hyperbolic, then $\operatorname{Hol}(X, Y)$ is locally compact with respect to the compact-open topology. For any point $p \in X$ and any compact subset $K \subset Y$, the family $F_{p, K}=\{f \in \operatorname{Hol}(X, Y) ; f(p) \in K\}$ is compact.

When $Y$ is not complete, we have the following theorem of Ascoli.

THEOREM 8.2. Let $Y$ be hyperbolic. Then a subfamily $F \subset \operatorname{Hol}(X, Y)$ is relatively compact if and only if, for each $p \in X,\{f(p) ; f \in F\}$ is a relatively compact subset of $Y$.

We say that a complex space $Y$ is taut modulo a subset $\Delta$ if, for every complex space $X$ and every sequence $\left\{f_{n}\right\}$ in $\operatorname{Hol}(X, Y)$, we have one of the following two possibilities:

(a) $\left\{f_{n}\right\}$ has a subsequence which converges in $\operatorname{Hol}(X, Y)$;

(b) for each compact $K \subset X$ and each compact $L \subset Y-\Delta$, there exists an integer $n_{0}$ such that $f_{n}(K) \cap L=\varnothing$ for $n \geqq n_{0}{ }^{3}$

According to Barth [2] it suffices to verify (a) and (b) for $X=D$ if $\Delta=\phi$.

If $Y$ is taut modulo the empty set, $Y$ is said to be taut. The concept of taut complex space was introduced by $\mathrm{Wu}[2]$ and Kaup [1] who used the term "hyperbolic". They made an extensive study of holomorphic maps into taut spaces. See also Reckziegel [1].

Concerning taut spaces and complete hyperbolic spaces, we have the following result of Kiernan [1] and Eisenman [2]:

THEOREM 8.3. A complex space $Y$ is taut if it is complete hyperbolic. It is hyperbolic if it is taut.

It is believed that $Y$ is complete hyperbolic if it is taut. This conjecture is supported by a number of parallel results for complete hyperbolic spaces and taut spaces; in addition to Wu [2] and Kaup [1], see also Barth [3].

To obtain a satisfactory generalization of Montel's theorem, we introduce another related concept. Given a relatively compact, open subset $Y$ of a complex space $Z$ and a (possibly empty) subset $\Delta$ of $Z$, we say that $Y$ is tautly imbedded in $Z$ modulo $\Delta$ if for every complex space $X$ and every sequence $\left\{f_{n}\right\} \subset \operatorname{Hol}(X, Y)$ we have one of the following two possibilities:

(a) $\left\{f_{n}\right\}$ has a subsequence which converges in $\operatorname{Hol}(\mathrm{X}, \mathrm{Z})$;

(b) for each compact $K \subset X$ and each compact $L \subset Z-\Delta$, there exists an integer $n_{0}$ such that $f_{n}(K) \cap L=\varnothing$ for $n \geqq n_{0}$.

We say that $Y$ is tautly imbedded in $Z$ if it is tautly imbedded modulo the empty set. If $\Delta$ is empty, (b) is impossible since $f_{n}(K) \cap L \neq \varnothing$ for $L=\bar{Y}$. Thus, $Y$ is tautly imbedded in $Z$ if and only if (a) holds, i.e., if and only if $\operatorname{Hol}(X, Y)$ is relatively compact in $\operatorname{Hol}(X, Z)$. The following result of Kiernan [2] may be considered as the principle of Montel's theorem on normal families.

\footnotetext{
${ }^{3} \mathrm{~T}$. Barth pointed out to me that this condition is stronger than the one in KiernanKobayashi [2] where $X$ is restricted to $D^{k}$.
} 
THEOREM 8.4. Let $Y$ be a relatively compact, open subset of a complex space $Z$. Then the following conditions are mutually equivalent:

(i) $Y$ is hyperbolically imbedded in $Z$;

(ii) $Y$ is tautly imbedded in $Z$, i.e., $\operatorname{Hol}(X, Y)$ is relatively compact in $\operatorname{Hol}(X, Z)$ for every complex space $X$;

(iii) For the unit disk $D, \operatorname{Hol}(D, Y)$ is relatively compact in $\operatorname{Hol}(X, Z)$;

(iv) Given a hermitian metric $d s_{Z}^{2}$ on $Z$, there is an everywhere positive continuous function $\varphi$ on $Z$ such that

$$
f^{*}\left(\varphi \cdot d s_{Z}^{2}\right) \leqq d s_{D}^{2} \quad \text { for all } f \in \operatorname{Hol}(D, Y),
$$

where $d s_{D}^{2}$ is the Poincaré metric of $D$.

When $\Delta$ is nonempty, our knowledge is less complete. First, we have to introduce two additional notions. We say that a relatively compact, open subset $Y$ in $Z$ is locally taut (resp. locally complete) in $Z$ if for each point $z \in \partial Y$ $(=\bar{Y}-Y)$ there exists a neighborhood $V_{z}$ of $z$ in $Z$ such that $V_{z} \cap Y$ is taut (resp. complete hyperbolic). Because of Theorem 8.3, if $Y$ is locally complete in $Z$, then it is locally taut in $Z$. For applications, it is useful to know the following simple criterion.

THEOREM 8.5. If $Z-Y$ is locally defined as the zeroes of a single holomorphic function, then $Y$ is locally complete (and hence locally taut) in $Z$.

For the proof, see Kiernan and Kobayashi [2]. The following result is also in the same paper. To facilitate the comparison, we state the corresponding result for $\Delta=\varnothing$ at the same time.

THEOREM 8.6. Let $Y$ be a relatively compact, open subset of a complex space $Z$ and $\Delta$ a subset of $Z$. Then we have the following diagrams of implications :

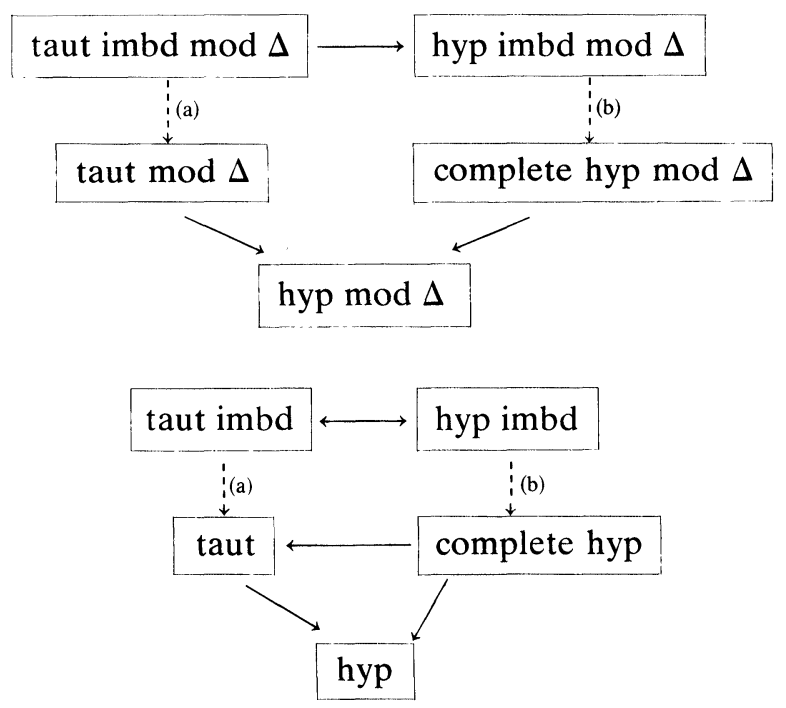


In these two diagrams, the dotted vertical arrows (a) (resp. (b)) are valid under the assumption that $Y$ is locally taut (resp. locally complete) in $Z$.

The equivalence between (i) and (iv) in Theorem 8.4 generalizes to the case of nonempty $\Delta$ (see Kiernan and Kobayashi [2]).

THEOREM 8.7. Let $Y, Z$ and $\Delta$ be as in Theorem 8.6. Then $Y$ is hyperbolically imbedded modulo $\Delta$ in $Z$ if and only if, given a hermitian metric $d s_{Z}^{2}$ on $Z$, there exists a continuous nonnegative function $\varphi$ on $Z$ such that

(a) $\varphi$ is strictly positive on $Z-\Delta$;

(b) $f^{*}\left(\varphi \cdot d s_{Z}^{2}\right) \leqq d s_{D}^{2}$ for all $f \in \operatorname{Hol}(D, Y)$.

At the beginning of this section, we mentioned the main result of Douady. In general, let $X$ be a compact complex space and $Y$ a complex space. Then we define a pseudo-distance $d$ in $\operatorname{Hol}(X, Y)$ by

$$
d(f, g)=\max _{x \in X} d_{Y}(f(x), g(x)) \quad \text { for } f, g \in \operatorname{Hol}(X, Y) .
$$

This definition is natural since Theorem 2.3 implies

$$
d_{Y^{k}}\left(y, y^{\prime}\right)=\max _{i}\left\{d_{Y}\left(y_{i}, y_{i}^{\prime}\right)\right\} \text { for } y=\left(y_{1}, \cdots, y_{k}\right), y^{\prime}=\left(y_{1}^{\prime}, \cdots, y_{k}^{\prime}\right)
$$

and since $Y^{k}=Y \times \cdots \times Y$ may be considered as the set of maps from a set of $X$ of $k$ points into $Y$. On the other hand, $\operatorname{Hol}(X, Y)$ is a complex space with many components according to Douady.

THEOREM 8.8. Let $X$ be a compact complex space and Y a complex space.

(1) The restriction of the pseudo-distance $d$ defined by (8.1) to each connected component $M$ of $\operatorname{Hol}(X, Y)$ coincides with the intrinsic pseudodistance $d_{M}$ of the complex space $M$;

(2) if $\mathrm{Y}$ is (complete) hyperbolic, each connected component $M$ of $\operatorname{Hol}(X, Y)$ is (complete) hyperbolic;

(3) if $Y$ is compact hyperbolic, $\operatorname{Hol}(X, Y)$ has only a finite number of connected components and each component is compact hyperbolic.

We want to explain a theorem of $\mathrm{H}$. Cartan which associates to each compact complex space $X$ a hyperbolic quotient complex space $X / R$ in a natural manner. This will allow us to reduce the study of $\operatorname{Hol}(X, Y)$ with compact $X$ and hyperbolic $Y$ to that of $\operatorname{Hol}(X / R, Y)$.

Let $\mathscr{C}$ be a class of complex spaces satisfying the following two conditions:

$\left(\mathrm{P}_{1}\right)$ the product of two spaces of $\mathscr{C}$ belongs to $\mathscr{C}$;

$\left(\mathrm{P}_{2}\right)$ if $X$ is a complex space and if, for each point $x$ of $X$, there exists a proper holomorphic map $f: X \rightarrow Y$ into space $Y$ of the class $\mathscr{C}$ such that the fibre $f^{-1}(f(x))$ contains $x$ as an isolated point, then $X$ belongs to $\mathscr{C}$.

If the spaces of $\mathscr{C}$ are compact, $\left(\mathrm{P}_{1}, \mathrm{P}_{2}\right)$ is equivalent to $\left(\mathrm{P}_{1}, \mathrm{P}_{2}^{\prime}, \mathrm{P}_{2}^{\prime \prime}\right)$, where

$\left(\mathrm{P}_{2}^{\prime}\right)$ any complex subspace of a space belonging to $\mathscr{C}$ belongs to $\mathscr{C}$; 
$\left(\mathrm{P}_{2}^{\prime \prime}\right)$ if $f: X \rightarrow Y$ is a ramified covering and if $Y$ belongs to $\mathscr{C}$, then $X$ belongs to $\mathscr{C}$.

Then the theorem of $\mathrm{H}$. Cartan states:

THeOREM 8.9. Let $\mathscr{C}$ be a class satisfying $\left(\mathrm{P}_{1}\right)$ and $\left(\mathrm{P}_{2}\right)$. Let $X$ be a complex space for which there exists at least one space $Y_{0}$ in $\mathscr{C}$ with a proper holomorphic map $X \rightarrow Y_{0}$. Let $R$ denote the equivalence relation defined on $X$ by all holomorphic maps of $X$ into the spaces $Y$ of the class $\mathscr{C}$. (That is, two points $x$ and $x^{\prime}$ of $X$ are equivalent if and only if $f(x)=f\left(x^{\prime}\right)$ for all $Y \in \mathscr{C}$ and all holomorphic maps $f: X \rightarrow Y$.) Then the space $X / R$ is a complex space belonging to $\mathscr{C}$ and the projection $X \rightarrow X / R$ is proper holomorphic with connected fibres.

From the construction of $X / R$ it follows that the diagram<smiles></smiles>

establishes a one-to-one correspondence between the holomorphic maps $f \in \operatorname{Hol}(X, Y)$ and the holomorphic maps $f^{\prime} \in \operatorname{Hol}(X / R, Y)$.

If $X$ is compact, the assumption in Theorem 8.9 is automatically satisfied since we can take any space $Y_{0}$ in $\mathscr{C}$ and a constant map $X \rightarrow Y_{0}$. We list several examples of classes $\mathscr{C}$ satisfying $\left(\mathrm{P}_{1}\right)$ and $\left(\mathrm{P}_{2}\right)$. (The first three are due to Cartan. The fourth was given by Kaup [1]. The remaining examples are analogous to the fourth.)

(1) the class $\mathscr{C}_{1}$ of all holomorphically complete spaces;

(2) the class $\mathscr{C}_{2}$ of all projective algebraic varieties;

(3) the class $\mathscr{C}_{3}$ of all compact algebraic varieties;

(4) the class $\mathscr{C}_{4}$ of all taut complex spaces;

(5) the class $\mathscr{C}_{5}$ of all hyperbolic complex spaces;

(6) the class $\mathscr{C}_{6}$ of all complete hyperbolic complex spaces;

(7) the class $\mathscr{C}_{7}$ of all compact hyperbolic complex spaces.

So if $X$ is a compact complex space and $R$ is the equivalence relation on $X$ defined by $\mathscr{C}_{5}$, then $X / R$ is defined and is compact hyperbolic. (Since $X$ is compact, we obtain the same result by using $\mathscr{C}_{4}, \mathscr{C}_{6}$ or $\mathscr{C}_{7}$.) The real question is how this equivalence relation is related to the equivalence relation defined by the pseudo-distance $d_{\mathrm{x}}$. (This problem will be discussed later.)

In $\S 3$, we introduced the concept of a Carathéodory-hyperbolic or $C$ hyperbolic complex space. Kaup [1] obtained many results on holomorphic maps into $C$-hyperbolic spaces. It is of interest to see if they can be generalized to hyperbolic spaces. We mention only one sample result of Kaup.

THEOREM 8.10. Let $X$ be a compact complex space and $Y$ a compact $C$-hyperbolic space. Then the set of holomorphic maps $f: X \rightarrow Y$ sending $a$ given point $x_{0} \in X$ to a given point $y_{0} \in Y$ is finite. 
Another result of the same nature is the following theorem of Borel and Narasimhan [1].

Theorem 8.11. Let $X$ and $Y$ be as in Theorem 8.10. Let $f, g \in \operatorname{Hol}(X, Y)$. If the two maps have the same image point $y_{0}=f\left(x_{0}\right)=g\left(x_{0}\right)$ at some point $x_{0}$ of $X$ and induce the same homomorphism $\pi_{1}\left(X, x_{0}\right) \rightarrow \pi_{1}\left(Y, y_{0}\right)$ between the fundamental groups, then $f=g$.

For mappings into a compact Riemann surface of higher genus, we have the following classical theorem of de Franchis (see Lang [1] and Samuel [1]).

TheOREM 8.12. Let $X$ be a compact complex space and $Y$ a compact Riemann surface of genus greater than 1 . Then the number of surjective meromorphic maps of $X$ onto $Y$ is finite. Moreover, there is a uniform upper bound on this number which depends on $X$ but not on $Y$.

The first statement of Theorem 8.12 was generalized by Kaup [1] to the case where $Y$ is a product of compact Riemann surfaces of genus greater than 1 . Lang [2] raised the question whether the theorem holds when $Y$ is compact hyperbolic. The question has been answered under a slightly different assumption on Y. We have (Kobayashi and Ochiai [4])

THEOREM 8.13. Let $X$ be a complex space and $Y$ a compact complex space of general type. Then the number of surjective meromorphic maps of $X$ onto $Y$ is finite.

For holomorphic or meromorphic maps into measure-hyperbolic manifolds, we have very few results. Given a complex space $X$ of dimension $n$, let $\Psi_{X}$ be the intrinsic pseudo-volume form defined in $\S 5$. Define the intrinsic total volume $\operatorname{Vol}(X)$ by

$$
\operatorname{Vol}(X)=\int_{X} \Psi_{X}
$$

If $X$ is a compact Riemann surface of genus $g \geqq 2$, then

$$
\operatorname{Vol}(X)=2 \pi(2 g-2)=-2 \pi \times(\text { Euler number of } X)
$$

since $\Psi_{X}$ has curvature -1 , i.e., Ric $\Psi_{X}=-\Psi_{X}$.

In general, we have (Kobayashi [4, pp. 22-25], Yau [2], Pelles [1])

THEOREM 8.14. Let $X$ and $Y$ be compact complex spaces of dimension $n$. Let $f \in \operatorname{Hol}(X, Y)$.

(1) If $\operatorname{Vol}(X)<\operatorname{Vol}(Y)$, then $f$ is degenerate everywhere on $X$.

(2) If $\operatorname{Vol}(X)<2 \cdot \operatorname{Vol}(Y)$ and $\Psi_{\mathrm{X}}>0$ on a nonempty open set, then $f$ is either degenerate everywhere or a biholomorphic map from $X$ onto $Y$.

In fact, the inequality $f^{*} \Psi_{\mathrm{Y}} \leqq \Psi_{\mathrm{X}}$ and the formula

$$
\operatorname{degree}(f)=\left(\int_{X} f^{*} \Psi_{Y}\right) / \operatorname{Vol}(Y)
$$


implies

$$
\text { degree }(f) \leqq \operatorname{Vol}(X) / \operatorname{Vol}(Y) \text { for } f \in \operatorname{Hol}(X, Y) \text {. }
$$

In the argument above, we can replace $\Psi_{X}$ and $\Psi_{Y}$ by $\tilde{\Psi}_{X}$ and $\tilde{\Psi}_{Y}$, respectively, and we set

$$
\tilde{\operatorname{Vol}}(X)=\int_{X} \tilde{\Psi}_{X}, \quad \tilde{\operatorname{Vol}}(Y)=\int_{Y} \tilde{\Psi}_{\mathrm{Y}} .
$$

Then we obtain

$$
\operatorname{degree}(f) \leqq \tilde{\operatorname{Vol}}(X) / \tilde{\operatorname{Vol}}(Y) \text { for } f \in \operatorname{Mer}(X, Y)
$$

provided that $\tilde{\Psi}_{Y}>0$ on a nonempty open subset. This shows that when $Y$ is compact measure-hyperbolic (resp. meromorphically measure-hyperbolic) we have an upper bound for the degree $f \in \operatorname{Hol}(X, Y)$ (resp. $f \in \operatorname{Mer}(X, Y)$ ), although we do not know if Theorem 8.13 generalizes to a compact (meromorphically) measure-hyperbolic space $\mathrm{Y}$ or not.

It is needless to say that Theorem 8.14 is valid for a meromorphic map $f: X \rightarrow Y$ if $\operatorname{Vol}(X)$ and $\operatorname{Vol}(Y)$ are replaced by $\operatorname{Vol}(X)$ and $\operatorname{Vol}(Y)$, respectively.

Even when $X$ and $Y$ are not necessarily compact, the result above is still valid (essentially) if $\operatorname{Vol}(X)<\infty$ and $\operatorname{Vol}(Y)<\infty$ and if $f$ is proper; for details, see Yau [2].

We note that Theorem 8.14 applied to Riemann surfaces implies that if $X$ and $Y$ are compact Riemann surfaces and if $2 \leqq \operatorname{genus}(X)<\operatorname{genus}(Y)$, then there is no holomorphic map $f: X \rightarrow Y$ other than the constant maps.

The reasoning above was used also by Kulle [2] in studying proper holomorphic maps of an open ball of $\boldsymbol{C}^{n}$ into itself.

9. Automorphisms and endomorphisms of complex spaces. Without going into the general theory of automorphisms of complex manifolds (cf. Kobayashi [8, Chapter III]), we state the two basic theorems we shall use in this section. The first theorem generalizes the classical theorem of $\mathbf{H}$. Cartan [3], [4] for bounded domains.

THEOREM 9.1. Let $X$ be a complex hyperbolic space and $\operatorname{Aut}(X)$ the group of holomorphic automorphisms of $X$. Then

(1) with respect to the compact-open topology, $\operatorname{Aut}(X)$ is a (real) Lie group of dimension at most $2 n+n^{2}$, where $n=\operatorname{dim} X$;

(2) the isotropy subgroup $\operatorname{Aut}(X)_{x}$ at each point $x \in X$ is compact;

(3) the Lie algebra aut $(X)$ of $\operatorname{Aut}(X)$ consists of complete (i.e., globally integrable) holomorphic vector fields. If $v \in \operatorname{aut}(X)$, then $\sqrt{ }-1 \cdot v$ is not in aut $(X)$, i.e., it is not globally integrable. In other words, no complex Lie group of positive dimension acts on $X$ effectively as a holomorphic transformation group.

This is essentially a metric-topological theorem and follows from the fact that $\operatorname{Aut}(X)$ is a closed subgroup of the group of isometries of $X$ with respect to $d_{X}$. For the details, see Kobayashi [4, pp. 70-72], [8, pp. 77-82] 
and Kaup [3]. For the basic properties of transformation groups on complex spaces (with singularities), see Kaup [4].

The second theorem is due to Bochner and Montgomery [1], [2]. (The generalization to compact complex spaces with singularities is due to Kerner [1].)

THEOREM 9.2. Let $X$ be a compact complex space. Then its automorphism group $\operatorname{Aut}(X)$ is a complex Lie group and its Lie algebra aut $(X)$ consists of all holomorphic vector fields on $X$.

Generally, if $X$ is a complex manifold without any restriction, $\operatorname{Aut}(X)$ can be too large to be a Lie group as in the case of $X=\boldsymbol{C}^{n}$ with $n \geqq 2$.

Let $\operatorname{Bim}(X)$ denote the group of bimeromorphic automorphisms of $X$. Theorem 3.11 implies the following

THEOREM 9.3. Let $X$ be a complete hyperbolic space. Then $\operatorname{Bim}(X)=\operatorname{Aut}(X)$.

It is not clear whether the completeness assumption is really necessary.

Making an essential use of Theorem 2.3, Konrad Peters [1] obtained the following theorem analogous to Cartan's [6] result.

Theorem 9.4. Let $X$ and $Y$ be two hyperbolic spaces and let Aut $^{0}(*)$ denote the identity component of $\operatorname{Aut}(*)$. Then the natural injection $\operatorname{Aut}(X) \times \operatorname{Aut}(Y) \rightarrow \operatorname{Aut}(X \times Y)$ induces an isomorphism $\operatorname{Aut}^{0}(X) \times \operatorname{Aut}^{0}(Y) \cong$ $\operatorname{Aut}^{0}(X \times Y)$.

He gives also conditions for $\operatorname{Aut}(X) \times \operatorname{Aut}(Y) \cong \operatorname{Aut}(X \times Y)$.

From Theorems 9.1 and 9.2 we obtain the following

THEOREM 9.5. Let $X$ be a compact hyperbolic complex space. Then $\operatorname{Aut}(X)(=\operatorname{Bim}(X))$ is finite.

We shall now discuss automorphisms of measure-hyperbolic spaces. As we stated at the very end of $\S 5$, we have

THEOREM 9.6. Let $X$ be a measure-hyperbolic complex space. Then no complex Lie group of positive dimension acts on X effectively as a holomorphic transformation group.

This generalizes only (3) of Theorem 9.1. We do not know if $\operatorname{Aut}(X)$ is a Lie group in this case.

From Theorems 9.2 and 9.6 we obtain a partial generalization of Theorem 9.5:

THEOREM 9.7. Let $X$ be a compact measure-hyperbolic complex space. Then $\operatorname{Aut}(X)$ is discrete. have

It is likely that $\operatorname{Aut}(X)$ is finite. At least, in an important special case we

THEOREM 9.8. Let $X$ be a compact complex space of general type. Then $\operatorname{Bim}(X)$ as well as $\operatorname{Aut}(X)$ is finite. 
This follows from Theorem 8.13. Theorem 9.8 has been obtained by Matsumura [1] purely algebraically making use of the theory of algebraic groups. A special case was considered earlier by Kobayashi [9] and Klaus Peters [1]:

THEOREM 9.9. Let $X$ be a compact complex manifold with ample canonical line bundle. Then $\operatorname{Aut}(X)$ is finite and $\operatorname{Bim}(X)=\operatorname{Aut}(X)$.

The fact that $\operatorname{Bim}(X)$ need not coincide with $\operatorname{Aut}(X)$ in Theorem 9.8 can be seen by blowing up a suitable point of a compact complex manifold $X$ with ample canonical line bundle.

We shall now consider holomorphic endomorphisms. The classical Schwarz lemma consists of two statements. If $f: D \rightarrow D$ is a holomorphic map with $f(0)=0$, then (i) $\left|f^{\prime}(0)\right| \leqq 1$ and (ii) the equality holds when and only when $f$ is biholomorphic. So far we have been concerned with only the first part of the Schwarz lemma. The following generalization of the second part was first proved by $\mathrm{H}$. Cartan [4], [5] and Carathéodory [3] for bounded domains. The generalization to hyperbolic manifolds is due to Kaup [3] and Wu [2]. (See also Kobayashi [4, p. 75].)

THeOREM 9.10. Let $X$ be a hyperbolic complex space and 0 a regular point of $X$. Let $f \in \operatorname{Hol}(X, X)$ and $f(0)=0$. Let $d f_{0}: T_{0}(X) \rightarrow T_{0}(X)$ denote the differential of $f$ at 0 . Then

(1) the eigenvalues of $d f_{0}$ have absolute value $\leqq 1$;

(2) if $d f_{0}$ is the identity linear transformation of $T_{0}(X)$, then $f$ is the identity transformation of $X$;

(3) if $\left|\operatorname{det} d f_{0}\right|=1$, then $f$ is biholomorphic.

A result similar to (3) of Theorem 9.10 is in Pelles [1], where $f$ is assumed to be measure-preserving. In the compact case, we can conclude that $f$ is biholomorphic without appealing to the distance $d_{\mathrm{x}}$. From formulae (8.6) and (8.8) we immediately obtain

THEOREM 9.11. Let $X$ be a compact complex space with $\operatorname{Vol}(X)=\int_{X} \Psi_{X}>0$ (resp. $\tilde{\operatorname{Vol}}(X)=\int_{X} \tilde{\Psi}_{X}>0$ ), the assumption being satisfied if $X$ is measurehyperbolic (resp. meromorphically measure-hyperbolic). Then every $f \in$ $\operatorname{Hol}(X, X)$ (resp. $f \in \operatorname{Mer}(X, X)$ ) is either degenerate everywhere or biholomorphic (resp. bimeromorphic).

Let $X$ be compact and $f \in \operatorname{Hol}(X, X)$. If $f$ is degenerate, $f(X)$ is a subspace of lower dimension. By iterating $f$, we obtain a decreasing sequence of complex subspaces:

$$
X \supset f(X) \supset f^{2}(X) \supset \cdots \supset f^{k}(X)=f^{k+1}(X)=\cdots
$$

which stops at $f^{k}(X)$ for some $k$. Then (Kaup [1]).

THEOREM 9.12. If $X$ is a compact hyperbolic space and if $f \in \operatorname{Hol}(X, X)$, then there is a positive integer $m$ such that $f^{2 m}=f^{m}$.

Let $g=f^{m}$ so that $g^{2}=g$. Then $X$ is a fibre space over $g(X)$ with projection $g$ and a holomorphic section $g: g(X) \rightarrow X$. If $X$ is nonsingular, so is $g(X)$. 
A similar result is not known for measure-hyperbolic spaces or even for spaces of general type.

10. Extension of holomorphic and meromorphic maps. Let $X$ be a complex space and $A$ a closed subset of $X$. We consider such analytic objects as holomorphic and meromorphic maps, complex subspaces, vector bundles, or sheaves all defined on $X-A$ and ask if they can be extended to $X$. Siu's recent monograph [1] gives a comprehensive survey of this general question. But we are interested here primarily in the problem of extending holomorphic or meromorphic maps.

First we consider the case where $A$ is a complex subspace of $X$. Let $f$ be a holomorphic or meromorphic map from $X-A$ into another complex space $Y$. If we impose no conditions on $Y$, it is, in general, impossible to extend $f$ to a map $f: X \rightarrow Y$ unless the dimension of $A$ is smaller than the minimum fibre dimension of $f$, i.e., smaller than $\operatorname{dim} f^{-1}(y)$ for all $y \in Y$ with $f^{-1}(y) \neq \varnothing$ (see Stein [1]). Consider, for instance, $X=\boldsymbol{C}^{n} \quad(n \geqq 2), \quad A=\{0\}$ and $\mathbf{Y}=\left(\boldsymbol{C}^{n}-\{0\}\right) / \boldsymbol{Z}$ (Hopf manifold), where $\boldsymbol{Z}$ acts on $\boldsymbol{C}^{n}-\{0\}$ by $(m, z) \in$ $\boldsymbol{Z} \times\left(\boldsymbol{C}^{n}-\{0\}\right) \rightarrow 2^{m} z \in \boldsymbol{C}^{n}-\{0\}$ (see Griffiths [2]). Then the natural projection $f: \boldsymbol{C}^{n}-\{0\} \rightarrow\left(\boldsymbol{C}^{n}-\{0\}\right) / \boldsymbol{Z}$ does not extend to $\boldsymbol{C}^{n}$ even as a meromorphic map.

The most basic extension theorem is that of Riemann which may be stated as follows:

THEOREM 10.1. Let $X$ be a normal complex space and A a complex subspace of codimension $\geqq 1$. Let $Y$ be a complex space for which $c_{Y}$ is a complete distance. Then every holomorphic map $f: X-A \rightarrow Y$ extends to a holomorphic map $f: X \rightarrow Y$.

This theorem says, in particular, that a bounded holomorphic function on $X-A$ extends to a bounded holomorphic function on $X$, and really nothing more. Another important extension theorem is that of Hartogs. We state it in the form generalized by Andreotti and Stoll [1].

THEOREM 10.2. Let $X$ be a normal complex space and A a subset of topological codimension $\geqq 3$ contained in a complex subspace of codimension $\geqq 1$. Let $Y$ be a complex space. Then every holomorphic map $f: X-A \rightarrow Y$ extends to a holomorphic map $f: X \rightarrow Y$ if one of the following two conditions is satisfied:

(1) $Y$ is a Stein space; $\tilde{Y}$.

(2) $X$ is a nonsingular complex manifold and $Y$ has a Stein covering space

When $Y=\boldsymbol{C}$, this is Hartogs' theorem. (It is usually assumed that $A$ is a complex subspace of codimension $\geqq 2$.)

In contrast to holomorphic maps into a Hopf manifold, we have the following well-known theorem for maps into projective algebraic varieties.

THEOREM 10.3. Let $X$ be a complex space and A a complex subspace of codimension $\geqq 2$. Let $Y$ be a projective algebraic variety. Then every meromorphic map $f: X-A \rightarrow Y$ extends to a meromorphic map $f: X \rightarrow Y$. 
It is not known if Theorem 10.3 still holds when $Y$ is a compact Kähler manifold. For partial results in this direction, see Griffiths [2] and Shiffman $[\mathbf{1}]$.

It is noteworthy that the extension theorems proved in $\$ 3$, Theorem 3.6 through Corollary 3.10, are all valid for a complex subspace $A$ of codimension $\geqq 1$ except Theorem 3.11 for which an assumption similar to that of Theorem 10.2 has to be made. In all these theorems (Theorems 3.6 through 3.11), $Y$ is a hyperbolic complex space. We have no extension theorems when $Y$ is merely (compact) measure-hyperbolic. But in an important special case, we have (Kobayashi and Ochiai [3], [4])

THEOREM 10.4. Let $X$ be a complex space and $A$ a complex subspace of codimension $\geqq 1$. Let $Y$ be an $n$-dimensional compact complex space of general type. Then every meromorphic map $f: X-A \rightarrow Y$ of maximal rank $n$ extends to a meromorphic map $f: X \rightarrow Y$.

In dimension $n \geqq 2$, every function holomorphic in a shell $X-A=\{z \in$ $\left.C^{n} ; 0<r<\|z\|<R\right\}$ extends to a holomorphic function in the ball $X=\{z \in$ $\left.C^{n} ;\|z\|<R\right\}$ (Hartogs' phenomenon). Now we want to state theorems generalizing this phenomenon.

Let $D$ be the unit disk in $\boldsymbol{C}$ and $A_{r}=\{z \in \boldsymbol{C} ; r<|z|<1\}$. We say that a complex space $X$ is disk-convex if every sequence $\left\{f_{n}\right\} \in \operatorname{Hol}(D, X)$ converges in $\operatorname{Hol}(D, X)$ whenever the sequence $\left\{f_{n} \mid A_{r}\right\} \in \operatorname{Hol}\left(A_{r}, X\right)$ converges in $\operatorname{Hol}\left(A_{r}, X\right)$ for some $r<1$. Shiffman [2] proved

THEOREM 10.5. Let $X$ be a domain in a Stein manifold $M$ and $E(X)$ its envelope of holomorphy. Let $Y$ be a disk-convex complex space. Then every holomorphic map $f: X \rightarrow Y$ extends to a holomorphic map $f: E(X) \rightarrow Y$.

Corollary 10.6. Let $X$ and $E(X)$ be as above. Then every holomorphic map $f: X \rightarrow Y$ extends to a holomorphic map $f: E(X) \rightarrow Y$ if $Y$ satisfies one of the following two conditions:

(1) $Y$ admits a hermitian metric of nonpositive holomorphic sectional curvature (or more generally, a differential metric of nonpositive curvature);

(2) $Y$ is complete hyperbolic (or more generally, taut).

The fact that $Y$ is disk-convex in case (1) was proved by Shiffman in the same paper. For a direct but similar proof of (1) of Corollary 10.6, see also Griffiths [2]. The fact that $Y$ is disk-convex in case (2) is proved in Kobayashi [4, p. 77]. A direct proof of (2) of Corollary 10.6 is in Fujimoto [2]. See also Kwack [3].

11. Defect relations. We shall briefly discuss the higher dimensional Nevanlinna theory. The main objective of the theory is to describe quantitatively the image of a holomorphic map $f: \boldsymbol{C}^{m} \rightarrow Y$ relative to the divisors of $Y$. At present, theory is in satisfactory form only in two cases: (i) $m=1$ and $Y=P_{n}(\boldsymbol{C})$ (holomorphic curves in $P_{n}(\boldsymbol{C})$ ), (ii) $m=n$ (the equidimensional case), although essential concepts of the theory are largely valid in the general case. This corresponds to the situation in the preceding sections 
where we could develop the theory of intrinsic pseudo-distances and pseudo-volume forms but could not obtain any significant result for intermediate dimensional intrinsic measures.

Let $Y$ be an $n$-dimensional projective algebraic manifold, and $S=$ $S_{1}+\cdots+S_{k}$ an effective divisor. Let $z^{1}, \cdots, z^{m}$ be the natural coordinate system in $\boldsymbol{C}^{m}$, and let $B_{\mathrm{r}}$ denote the closed ball of radius $r, B_{\mathrm{r}}=\{z \in$ $\left.\boldsymbol{C}^{m} ;\|z\|<r\right\}$, where $\|z\|^{2}=\left|z^{1}\right|^{2}+\cdots+\left|z^{m}\right|^{2}$. Define a $(1,1)$-form $\psi$ on $\boldsymbol{C}^{m}$ by

$$
\psi=(\sqrt{ }(-1) / 2 \pi) \partial \bar{\partial} \log \|z\|^{2} .
$$

Given a nondegenerate holomorphic map $f: \boldsymbol{C}^{m} \rightarrow Y$, we set

$$
\begin{aligned}
& n(S, \rho)=\int_{f^{-1} S \cap B_{\rho}} \psi^{m-1}, \\
& N(S, r)=\int_{0}^{r} n(S, \rho) \rho^{-1} d \rho \quad \text { (counting function). }
\end{aligned}
$$

Let $L$ be a line bundle over $Y$ and let $\omega$ be a closed real $(1,1)$-form representing the Chern class of $L$. Set

$$
\begin{aligned}
t(L, \rho) & =\int_{B_{\rho}} f^{*} \omega \wedge \psi^{m-1} \\
T(L, r) & =\int_{0}^{r} t(L, \rho) \rho^{-1} d \rho \quad \text { (order function). }
\end{aligned}
$$

Now we consider the case of a holomorphic curve in $P_{n}(\boldsymbol{C})$. We let $Y=P_{n}(\boldsymbol{C}), S=$ a hyperplane $H$ in $P_{n}(\boldsymbol{C})$, and $L=$ the line bundle defined by the divisor $H$. Choosing the natural hermitian metric in $L$, we may assume that $\omega$ is the Kähler form associated to the Fubini-Study metric of $P_{n}(\boldsymbol{C})$ normalized in such a way that $\int_{P_{n}(\boldsymbol{C})} \omega^{n}=1$. We assume that $f: \boldsymbol{C} \rightarrow P_{n}(\boldsymbol{C})$ is nondegenerate in the sense that $f(\boldsymbol{C})$ is not contained in any hyperplane of $P_{n}(\boldsymbol{C})$. Now, $B_{r}$ is the closed disk of radius $r$ in $\boldsymbol{C}$. Definition (11.1) means that $f\left(B_{\rho}\right)$ meets the hyperplane $H$ exactly $n(H, \rho)$ times, counted with multiplicity. Since the line bundle $L$ and the Fubini-Study metric of $P_{n}(\boldsymbol{C})$ are independent of the choice of a hyperplane $H$, we denote $t(L, \rho)$ simply by $t(\rho)$. Then $t(\rho)=\int_{B_{\rho}} f^{*} \omega$ is the area of $f\left(B_{\rho}\right)$ measured by the FubiniStudy metric of $P_{n}(\boldsymbol{C})$. Similarly, we write $T(r)$ for $T(L, r)$. The counting function $N(H, r)$ measures how frequently $f\left(B_{r}\right)$ meets $H$. The justification for using $N(H, r)$ rather than $n(H, r)$ to measure this frequency comes from the equality

$$
T(r)=\int N(H, r) d H,
$$

where the right-hand side is the integral of $N(H, r)$ over the dual projective space with respect to the natural measure $d H$. The image $f\left(B_{r}\right)$ may meet some hyperplanes more often than others, and (11.5) expresses the fact that $T(r)$ is the average frequency. The Nevanlinna inequality (a consequence of the so-called First Main Theorem) states:

$$
N(H, r)<T(r)+C,
$$


where $C$ is a constant depending on $H$ but independent of $r$. This inequality implies that the defect $\delta(H)$ defined by

$$
\delta(H)=1-\limsup _{r \rightarrow \infty}(N(H, r) / T(r))
$$

lies between 0 and 1 . The equality $\delta(H)=0$ means that $H$ meets $f(\boldsymbol{C})$ as often as any other hyperplane does. If $\delta(H)>0$, then $H$ meets $f(\boldsymbol{C})$ less often than the average. In particular, if $H$ does not meet $f(\boldsymbol{C})$ at all, we have $\delta(H)=1$. The most important result in Nevanlinna theory is the following defect relation, proved by Nevanlinna for $n=1$ and by Ahlfors for an arbitrary $n$.

THEOREM 11.1 Let $H_{1}, \cdots, H_{k}$ be hyperplanes in general position in $P_{n}(\boldsymbol{C})$. Let $f: C \rightarrow P_{n}(C)$ be a nondegenerate holomorphic curve. Then $\sum_{i} \delta\left(H_{i}\right) \leqq n+1$.

For the proof of Theorem 11.1 and the detail of the foregoing discussion, see $\mathrm{H}$. Weyl and $\mathrm{J}$. Weyl [1] and $\mathrm{Wu}[3]$. The most satisfactory proof from the differential geometric viewpoint is in Chern [4], where the defect relation is proved for $n=2$ but everything else is done for an arbitrary $n$, and in Cowen and Griffiths [1]. In the latter, one finds that metrics of negative curvature play an essential role as in the theory of intrinsic pseudodistances. Chern's proof of the defect relation was generalized for an arbitrary $n$ by H. Yamaguchi in 1972 (unpublished).

Consequences of Theorem 11.1 will be discussed in the next section in relation to the principle of the little Picard theorem established earlier (see Theorem 3.5).

The defect relation in the equidimensional case was first obtained by Carlson and Griffiths [1]. We present it in the form generalized by Sakai [2], [3]. Let $Y$ be an $n$-dimensional projective algebraic manifold and $f: \boldsymbol{C}^{n} \rightarrow Y$ be a nondegenerate holomorphic map. Given a divisor $S$ of $Y$, the counting function $N(S, r)$ is defined by (11.2). Given a line bundle $L$ over $Y$, the order function $T(L, r)$ is defined by (11.4). The line bundle defined by $S$ is denoted by $[S]$. Then the defect is defined by

$$
\delta(S)=1-\limsup _{r \rightarrow \infty}(N(S, r) / T([S], r)) .
$$

We introduce a few more quantities:

$$
n_{1}(\rho)=\int_{\left(J_{f}\right) \cap B_{\rho}} \psi^{n-1}
$$

where $\left(J_{f}\right)$ denotes the divisor of $\boldsymbol{C}^{n}$ given as the zero set of the Jacobian $\boldsymbol{J}_{f}$ of $f$. We set

$$
\begin{aligned}
N_{1}(r) & =\int_{0}^{r} n_{1}(\rho) \rho^{-1} d \rho, \\
\gamma_{1}([S]) & =\liminf _{r \rightarrow \infty}\left(N_{1}(r) / T([S], r)\right) .
\end{aligned}
$$

Then 
THEOREM 11.2. Let $S_{1}, \cdots, S_{k}$ be nonsingular divisors on a projective algebraic manifold $Y$ such that $S=S_{1}+\cdots+S_{k}$ has only normal crossings. Let $K$ denote the canonical line bundle of $Y$. Assume that there exist integers $q_{0}, \cdots, q_{k}$ such that the line bundle $L$ defined by $L=K^{q_{0}}\left[S_{1}\right]^{q_{1}} \cdots\left[S_{k}\right]^{q_{k}}$ satisfies

$$
\limsup _{m \rightarrow \infty} m^{-n} \operatorname{dim} \Gamma\left(L^{m}\right)>0 .
$$

Let $f: \boldsymbol{C}^{n} \rightarrow Y$ be a nondegenerate holomorphic map $(n=\operatorname{dim} Y)$. Then

$$
\delta(S)+\gamma_{1}([S]) \leqq \limsup _{r \rightarrow \infty}(-T(K, r) / T([S], r)) .
$$

This is only one of the results in Sakai [2], [3]. We recall that (11.12) is similar to (7.5) used in defining the concept of a manifold of general type. In terms of the concept of L-dimension of Iitaka [1], (11.12) means that the $L$-dimension of $Y$ is equal to the complex dimension $n$ of $Y$. If $L$ is ample, (11.12) is automatically satisfied. Carlson and Griffiths [1] obtained the result above in the case where $\left[S_{1}\right]=\cdots=\left[S_{k}\right]$ and the line bundle $L=K\left[S_{1}\right]$ is ample. We mention two consequences of Theorem 11.2 (see Sakai [2]).

Corollary 11.3. Let $Y=P_{n}(\mathbf{C})$ and let $S_{1}, \cdots, S_{k}$ be a nonsingular hypersurface of degree $d_{1}, \cdots, d_{k}$ such that $S=S_{1}+\cdots+S_{k}$ has normal crossings. If $f: \boldsymbol{C}^{n} \rightarrow P_{n}(\boldsymbol{C})$ is a nondegenerate holomorphic map, then $\sum_{i=1}^{k} d_{i} \cdot \delta\left(S_{i}\right) \leqq n+1$.

This follows from the fact that if $H$ is any hyperplane of $P_{n}(C)$, then $K=[H]^{-(n+1)}$ and $\left[S_{i}\right]=[H]^{d_{i}}$ so that $-T(K, r) / T([S], r)=(n+1) / d$, where $d=\sum_{i=1}^{k} d_{i}$. The following corollary can be derived from the special case considered by Carlson and Griffiths [1].

Corollary 11.4. Let $H_{1}, \cdots, H_{k}$ be hyperplanes in general position in $P_{n}(\boldsymbol{C})$. If $f: \boldsymbol{C}^{n} \rightarrow P_{n}(\boldsymbol{C})$ is a nondegenerate holomorphic map, then $\sum_{i=1}^{k} \delta\left(H_{i}\right) \leqq n+1$.

The proof of Theorem 11.2 involves volume forms with negative Ricci form, and it is simpler than the proof of Theorem 11.1 which in essence has to do with metrics of negative curvature.

For Nevanlinna theory for holomorphic maps of an affine algebraic manifold into a projective algebraic manifold of the same dimension, see Griffiths and King [1]. We have seen that the theory of intrinsic measures is applicable not only to holomorphic maps but also to meromorphic maps. Similarly, the equidimensional Nevanlinna theory generalizes to meromorphic maps (see Shiffman [3]).

\section{Examples and applications.}

EXAMPLE 1 . Since $d_{\mathbf{c}}=0$, we have $d_{\mathrm{G}}=0$ if $G$ is a complex Lie group. If $X$ is a complex space with a holomorphic map $f: G \rightarrow X$ such that $f(G)$ is dense in $X$, then $d_{X}=0$. In particular, if a complex Lie group $G$ acts on $X$ with a dense orbit, then $d_{X}=0$. If $X$ is a compact homogeneous complex manifold, e.g., $P_{n}(\boldsymbol{C})$, a hyperquadric, or a complex torus, then $d_{X}=0$. If two 
complex spaces $X$ and $Y$ are bimeromorphic to each other, then $d_{X}=0$ if and only if $d_{Y}=0$; this is because every meromorphic map $f: D \rightarrow X$ is holomorphic. Hence, $d_{X}=0$ if $X$ is a rational variety. If $f: Y \rightarrow X$ is a surjective meromorphic map and $d_{Y}=0$, then $d_{X}=0$. Hence, $d_{X}=0$ if $X$ is unirational (i.e., if there is a surjective meromorphic map $P_{n}(C) \rightarrow X$ with $n=\operatorname{dim} X$ ). If $X$ is a generalized Kummer variety (i.e., if there is a surjective meromorphic map from a complex torus $T$ with $\operatorname{dim} T \geqq \operatorname{dim} X$ ), then $d_{X}=0$. Since $\boldsymbol{C}^{n}-\{0\}$ has the trivial intrinsic pseudo-distance, for a Hopf surface $X$ we have $d_{X}=0$.

EXAMPLE 2. If $X$ is a complex space on which a complex Lie group acts, then $\Psi_{X}=0$ (see the end of $\S 5$ ). If $Y$ is bimeromorphic to such a space $X$, then $\tilde{\Psi}_{Y}=0$. If $X$ is a compact homogeneous complex manifold, then $\Psi_{X}=0$. If $X$ is a rational or unirational variety or a generalized Kummer variety, then $\tilde{\Psi}_{X}=0$ as above. If $X$ is a Hopf surface, then $\Psi_{X}=0$. If $X=C \times D^{n-1}$, then $\Psi_{X}=0$. Hence, if $X$ is an elliptic surface, then $\tilde{\Psi}_{X}=0$. If $X=P_{1}(C) \times D^{n-1}$, then $\Psi_{X}=0$. Hence, if $X$ is a ruled surface, then $\tilde{\Psi}_{X}=0$.

EXAMPLE 3. Let $X$ be a complete Kähler manifold with positive semidefinite Ricci tensor. Then $c_{X}=0$ (Yau [3]). (This is of interest only when the Ricci tensor is not strictly positive and bounded away from zero because $c_{X}=0$ trivially if $X$ is compact.) The next two examples will justify the term "hyperbolic space."

EXAmple 4. Let $X$ be the complex line $\boldsymbol{C}$ minus a discrete set of points. By Theorem 2.4 and Example 1, $c_{X}=0$. The situation is quite different for $d_{x}$. If we delete only one point, say the origin, then we still have $d_{c^{*}}=0$ by Example 1 since $\boldsymbol{C}^{*}$ is a complex Lie group. But as soon as we delete two points, say 0 and 1 , the resulting space $X$ becomes complete hyperbolic, and by Theorem 3.5 every holomorphic map $f: \mathbf{C} \rightarrow \boldsymbol{C}-\{0,1\}$ is constant (the little Picard theorem). The fact that $\boldsymbol{C}-\{0,1\}$ is complete hyperbolic can be seen from Theorem 3.3 and from the fact that the upperhalf-plane (which is biholomorphic to the disk $D$ ) is the universal covering space of $\boldsymbol{C}-\{0,1\}$, the projection being given by the modular function $\lambda$. This is the way the Picard theorem was originally proved. This proof shows also that the intrinsic distance $d_{\mathrm{X}}$ for $\boldsymbol{X}=\boldsymbol{C}-\{0,1\}$ comes from a metric of constant negative curvature -1 . But to see that $\boldsymbol{C}-\{0,1\}$ is hyperbolic, it suffices to construct a hermitian metric of negatively bounded curvature (Theorem 6.4). Such a metric was explicitly constructed in Robinson [1] (cf. the construction in Grauert and Reckziegel [1], reproduced in Kobayashi [4]). This is a differential geometric "elementary" proof of the little Picard theorem. We do not give here the explicit expression of such a metric since we shall give a more general result in another example. If we consider $\boldsymbol{C}-\{0,1\}$ as the complement of three points, say $\infty, 0,1$, in the Riemann sphere $P_{1}(C)$, then $\boldsymbol{C}-\{0,1\}$ is hyperbolically imbedded in $P_{1}(\boldsymbol{C})$ in the sense of $\S 3$. In this particular case, condition (b) for the hyperbolic imbedding is trivially satisfied although in many cases this condition is difficult to verify. Hence, from Theorem 3.6 we obtain the classical great Picard theorem: every holomorphic map $f: D^{*} \rightarrow \boldsymbol{C}-\{0,1\}$ extends to a holomorphic map $f: D \rightarrow$ 
$P_{1}(\boldsymbol{C})$. The implication (i) $\rightarrow$ (ii) of Theorem 8.4 applied to the hyperbolically imbedded $\boldsymbol{C}-\{0,1\}$ in $\boldsymbol{P}_{1}(\boldsymbol{C})$ yields the classical theorem of Montel. It should be noted that the complete hyperbolicity of $\boldsymbol{C}-\{0,1\}$ follows from the fact that $\boldsymbol{C}-\{0,1\}$ is hyperbolically imbedded (see Theorem 3.13) although it may be also derived from the fact that the hermitian metric of negatively bounded curvature constructed by Grauert-Reckziegel is a complete metric (see Theorem 6.4).

EXAMPLE 5. Let $X$ be a compact Riemann surface of genus $g$. Since $X$ is compact, $c_{X}=0$ irrespective of the genus $g$. If $g=0$ or 1 , then $d_{X}=0$ by Example 1. If $g \geqq 2$, then the disk $D$ is the universal covering space of $X$, and $X$ is complete hyperbolic. The intrinsic distance $d_{\mathrm{x}}$ comes from a metric of constant negative curvature -1 . If we are only interested in knowing that $X$ is complete hyperbolic when $g \geqq 2$, we have only to construct a hermitian metric with negative curvature explicitly using linearly independent holomorphic 1-forms $\omega_{1}, \cdots, \omega_{\mathrm{g}}$ as in Grauert and Reckziegel [1] and then apply Theorem 6.4 (see also Kobayashi $[4$, p. 12]). The existence of such a hermitian metric may be also derived from the fact that the canonical line bundle of $X$ is ample if $g \geqq 2$ and from the implication (1) $\rightarrow(2)$ of Theorem 7.1 .

We shall now consider examples of domains in $C^{\mathfrak{n}}$.

Example 6. Let $\Omega$ be an open cone in $\boldsymbol{R}^{n}$ and let $T_{\Omega}=\left\{z=x+i y \in \boldsymbol{C}^{n} ; y \in\right.$ $\Omega\}$ be the tube domain defined by $\Omega$. Then $T_{\Omega}$ is hyperbolic if and only if the cone $\Omega$ contains no whole straight lines. (Such a tube domain is called a Siegel domain of the first kind in the terminology of Pyatetzki-Šapiro [1].) It is complete hyperbolic if and only if the cone $\Omega$ (without straight lines) is convex. It is indeed finitely compact (and hence Cauchy-complete) with respect to its Carathéodory distance $c_{T_{\Omega}}$ if $\Omega$ is convex. The same result holds even for any Siegel domain of the second kind; see Sibony [1].

Example 7. Let $X$ be a bounded symmetric domain in $\boldsymbol{C}^{n}$. Then $c_{X}=d_{X}$ (see Kobayashi [4]). From the homogeneity we can conclude that $X$ is complete hyperbolic. This is also a special case of the example above, Siegel domain of the second kind.

The following interesting example is due to Sibony [1], [2].

EXAMPLE 8. Let $\left\{a_{k}\right\}$ be a discrete sequence of points in the unit disk $D$ and $\left\{\lambda_{k}\right\}$ a sequence of positive real numbers with $\sum \lambda_{k}<\infty$. We define functions $\varphi$ and $\Phi$ on $D$ by

$$
\varphi(z)=\sum_{k} \lambda_{k} \cdot \log \frac{\left|z-a_{k}\right|}{2}, \quad \Phi(z)=\exp (\varphi(z))
$$

and a domain $X$ in $D \times C$ by

$$
X=\{(z, w) \in D \times C ;|w|<\exp (-\Phi(z))\} .
$$

The function $|w| \exp (\Phi)$ is plurisubharmonic and $X$ is a pseudo-convex domain contained in the bidisk $D^{2}$. In particular, $X$ is a domain of holomorphy. Assume further that the sequence $\left\{a_{k}\right\}$ was chosen in such a 
way that every point of the boundary of $D$ is a nontangential limit of a suitable subsequence of $\left\{a_{k}\right\}$. Then $X$ has the following properties:

(1) $X$ is a Runge domain, i.e., every holomorphic function on $X$ can be uniformly approximated on an arbitrary compact set in $X$ by polynomials of $(z, w)$

(2) $X$ is convex with respect to the family of bounded holomorphic functions on $X$;

(3) Every bounded holomorphic function on $X$ extends to a bounded holomorphic function on $D^{2}$;

(4) $X$ is not Cauchy-complete with respect to $c_{X}$.

This example shows that the converse of (e) of Theorem 4.1 is not true in general. It shows also that an $H^{\infty}$-convex domain need not be a domain of bounded holomorphy. On the other hand, we have another example due to Sibony [1], [2]:

Example 9. Let $X=\left\{(z, w) \in \boldsymbol{C}^{2} ;|w|<|z|<1\right\}$. Then $X$ is a domain of bounded holomorphy. Let $X^{\prime}=\left\{(z, u) \in \boldsymbol{C}^{2} ; 0<|z|<1,|u|<1\right\}$. The map $(z, u) \rightarrow(z, u z)$ gives a biholomorphic correspondence between $X^{\prime}$ and $X$. It follows that $X$ is not $H^{\infty}$-convex. Clearly, $X$ is complete hyperbolic but is not Cauchy-complete with respect to $c_{X}$.

The following related example is due to Ahern and Schneider [1]:

EXAMPLE 10. Let $\left\{a_{k}\right\}$ be a sequence of positive numbers converging monotonically to 0 . Let $\left\{c_{k}\right\}$ be another sequence of positive numbers such that the closed disks of radius $c_{k}$ about $a_{k}$ are mutually disjoint and such that $\sum c_{k}\left(a_{k}-c_{k}\right)^{-1}<\frac{1}{2}$. Let $X$ be the Riemann sphere with 0 and the union of these closed disks removed. This is a one-dimensional example of an $H^{\infty}$ convex domain which is not finitely compact with respect to the Carathéodory distance.

EXAMPLE 11. An often-asked question is whether there exists a hyperbolic manifold $X$ which cannot be written as the quotient of a bounded domain by a properly discontinuous group of automorphisms. The following example by Kaup is such a noncompact hyperbolic manifold. Consider $\boldsymbol{C}^{2}-\{0\}$ as a principal bundle over $P_{1}(\boldsymbol{C})$ with group $\boldsymbol{C}^{*}$. Let $L$ be the associated line bundle over $P_{1}(\boldsymbol{C})$; it may be also obtained from $\boldsymbol{C}^{2}$ by blowing up the origin. (The origin blows up to the zero section of $L$.) Let $B$ be the set of elements of $L$ of length less than 1 with respect to the natural hermitian fibre metric; it may be obtained from the unit ball in $\boldsymbol{C}^{2}$ by blowing up the origin. It is a unit disk bundle over $P_{1}(\boldsymbol{C})$. Choose any three points in $P_{1}(\boldsymbol{C})$ and remove the closed disk of radius $\frac{1}{2}$ from the fibre over each of these three points. Then the resulting space $X$ is a simply connected complete hyperbolic manifold which is not $C$-hyperbolic in the sense of $\S 3$.

The following example is due to Green [2].

EXAMPLE 12. Let $C$ be a nonsingular algebraic curve in $P_{2}(\boldsymbol{C})$ and let $C^{*}$ be the dual curve in the dual projective plane $P_{2}^{*}(\boldsymbol{C}) ; C^{*}$ consists of lines in $P_{2}(\boldsymbol{C})$ which are tangent to $C$. We define a branched covering space $X$ of $P_{2}^{*}(\boldsymbol{C})$ with branch locus $C^{*}$ as follows. Let $C^{*}$ be given by the vanishing of a homogeneous polynomial $f\left(w_{0}, w_{1}, w_{2}\right)$ in $P_{2}^{*}(\boldsymbol{C})$. Then define $X \subset P_{3}(\boldsymbol{C})$ by 
the vanishing of the polynomial $w_{3}^{d}-f\left(w_{0}, w_{1}, w_{2}\right)$, where $d$ is the degree of $f$. (It is known that $d=k(k-1)$ if the degree of $C$ is $k$.) Then $X$ is simply connected by a theorem of Lefschetz. Green shows that if $k \geqq 4$, then $X$ is hyperbolic. But $X$ has singularities.

In Theorem 7.1 we saw that a compact complex manifold with ample canonical line bundle admits a volume form with negatively bounded Ricci form and hence is measure-hyperbolic. We shall give concrete examples of such manifolds.

EXAMPLE 13. Let $X$ be a nonsingular hypersurface in $P_{n+1}(C)$ of degree $d$. If we denote the positive generator of $H^{2}\left(P_{n+1}(\boldsymbol{C}) ; \boldsymbol{Z}\right)=\mathbf{Z}$ by $h$, then the first Chern class $c_{1}(X)$ of $X$ is given by

$$
c_{1}(X)=\left.(n+2-d) h\right|_{x},
$$

(see Hirzebruch [1, Appendix 1]). Hence, if $d>n+2$, then the canonical line bundle of $X$ is ample. More generally, if $X_{1}, \cdots, X_{r}$ are nonsingular hypersurfaces of $P_{n+r}(C)$ intersecting transversally, then $X=X_{1} \cap \cdots \cap X_{r}$ is a nonsingular submanifold of dimension $n$ and is called a complete intersection submanifold of $P_{n+r}(\boldsymbol{C})$. The degree $d$ of $X$ is defined to be the sum of the degrees of $X_{1}, \cdots, X_{r}$. Then $c_{1}(X)=\left.(n+r+1-d) h\right|_{X}$, where $h$ is the positive generator of $H^{2}\left(P_{n+r}(\boldsymbol{C}) ; Z\right)$. Hence, if $d>n+r+1$, then the canonical line bundle of $X$ is ample. (In fact, it is very ample.) In general, given a closed real $(1,1)$-form $\gamma$ on $X$ representing $c_{1}(X)$, we can find a volume form $v_{x}$ such that $\gamma=4 \pi{ }^{\prime}$ Ric $v_{x}$. But it is not known if such a volume form comes from a Kähler metric of $X$ (Calabi's problem).

EXAMPLE 14. If $z^{0}, z^{1}, \cdots, z^{n+1}$ is a homogeneous coordinate system for $P_{n+1}(\boldsymbol{C})$, then the hypersurface defined by $\sum_{i=0}^{n+1}\left(z^{i}\right)^{d}=0$ is called the Fermat variety of degree $d$ and will be denoted by $F(d)$. By the assertion made in Example 13, $F(d)$ has (very) ample canonical line bundle if $d>n+2$. If $n \geqq 2$, then the Fermat variety contains a rational curve

$$
\begin{gathered}
z^{0}=u, \quad z^{1}=\eta \cdot u, \quad z^{2}=v, \quad z^{3}=\eta \cdot v \quad(\eta=d \text { th root of }-1) \\
z^{4}=\cdots=z^{n+1}=0,
\end{gathered}
$$

and hence is not hyperbolic. For $n=1, F(d)$ is hyperbolic if $d>3$.

EXAMPLE 15. Let $Y$ be the complement of $n+2$ hyperplanes in general position in $P_{n}(\boldsymbol{C})$. We shall show that $Y$ admits a volume form $v_{Y}$ with negatively bounded Ricci form. We identify $P_{n}(\boldsymbol{C})$ with the Fermat variety $F(1)$ of degree 1 in $P_{n+1}(\boldsymbol{C})$ defined in Example 14. Thus $P_{n}(\boldsymbol{C})$ is the hyperplane in $P_{n+1}(\boldsymbol{C})$ defined by $z^{0}+z^{1}+\cdots+z^{n+1}=0$. Let $H_{i}$ be the hyperplane in $P_{n+1}(\boldsymbol{C})$ defined by $z^{i}=0$. Then without loss of generality we may assume that $Y=F(1)-\bigcup_{i=0}^{n+1} H_{i}$. The mapping $f_{d}: P_{n+1}(\boldsymbol{C}) \rightarrow P_{n+1}(\boldsymbol{C})$ defined by

$$
f_{d}\left(z^{0}, z^{1}, \cdots, z^{n+1}\right)=\left(\left(z^{0}\right)^{d},\left(z^{1}\right)^{d}, \cdots,\left(z^{n+1}\right)^{d}\right)
$$

induces a covering projection $F(d)-\bigcup H_{i} \rightarrow F(1)-\bigcup H_{i}=Y$. Now let $d>n+2$. Set $\tilde{Y}=F(d)-\bigcup H_{i}$. By Example 14, the intrinsic pseudo-volume form $\tilde{\Psi}_{F(d)}$ is positive, and $F(d)$ is (meromorphically) measure-hyperbolic. 
Hence $\tilde{\Psi}_{\tilde{Y}}>0$ and $\tilde{Y}$ is also (meromorphically) measure-hyperbolic. Since $F(d)$ is a covering space of $F(1)$ branched over $F(1) \cap\left(\cup H_{i}\right)$, it follows that $\tilde{Y}$ is an unbranched covering space of $Y$. By (7) of Theorem 5.1, $\tilde{\Psi}_{Y}>0$ and $Y$ is (meromorphically) measure-hyperbolic. This is probably the simplest way to see that $Y$ is (meromorphically) measure-hyperbolic. By Theorem 5.6, every meromorphic map $f: \boldsymbol{C}^{n} \rightarrow Y$ is everywhere degenerate. By setting $n=1$, we see that $P_{1}(\boldsymbol{C})$ with three points removed is hyperbolic and obtains the classical little Picard theorem (see Example 4). If we want to have a smooth volume form $v_{Y}$ with negatively bounded Ricci form, we have to construct a volume form $v_{\tilde{Y}}$ on $\tilde{Y}$ which is invariant under the covering transformations and has negatively bounded Ricci form. Let $\zeta$ be the primitive $d$ th root of 1 and let $G$ be the finite group of transformations of $F(d)$ consisting of transformations

$$
\left(z^{0}, \cdots, z^{n+1}\right) \rightarrow\left(\zeta^{k_{0}} z^{0}, \cdots, \zeta^{k_{n+1}} z^{n+1}\right) .
$$

Take any volume form $v_{F(d)}^{\prime}$ on $F(d)$, and let $\omega$ be a $G$-invariant closed $(1,1)$-form cohomologous to Ric $v_{F(d)}^{\prime}$; to obtain such a form $\omega$, restrict the Kähler form of $P_{n+1}(\boldsymbol{C})$ to $F(d)$ and multiply it by a suitable (negative) constant. Then there is a volume form $v_{F(d)}$, unique up to a constant factor, such that $\omega=\operatorname{Ric} v_{F(d)}$. This form is invariant by $G$. We have now only to set $v_{\tilde{Y}}=\left.v_{F(d)}\right|_{\tilde{Y}}$. We note that the volume of $Y$ measured by $v_{Y}$ is finite; in fact,

$$
\int_{Y} v_{Y}=\frac{1}{|G|} \int_{F(d)} v_{F(d)}
$$

We shall now examine the intrinsic pseudo-distance of the same example to obtain finer results.

EXAMPLE 16. As in Example 15, let $Y$ be the complement of $n+2$ hyperplanes in general position in $P_{n}(\boldsymbol{C})=F(1)$. (We use the same notation as in Example 15.) For each subset $I=\left\{j_{1}, \cdots, j_{k}\right\}$ of $\{0,1, \cdots, n+1\}$ consisting of at least two but no more than $n$ indices, we define a hyperplane $\Delta_{I}$ of $P_{n}(\boldsymbol{C})$ by $z^{j_{1}}+\cdots+z^{j_{k}}=0$, which is called the diagonal hyperplane corresponding to the index set $I$. We set $\Delta=\bigcup_{I} \Delta_{I}$. Then $Y$ is complete hyperbolic modulo $\Delta$. This is a reformulation of the result of Bloch [1]. A more precise result follows from the main theorem of Cartan [1]. Namely, Y is tautly (and hence hyperbolically) imbedded in $P_{n}(\boldsymbol{C})$ modulo $\Delta$. (For these reformulations of the results of Bloch and Cartan, see Kiernan and Kobayashi [2].) Applying Theorem 3.5 to this example, we can conclude that every nonconstant holomorphic map $f: \boldsymbol{C}^{m} \rightarrow Y$ sends $\boldsymbol{C}^{m}$ into one of its diagonal hyperplanes. This is the classical result of E. Borel [1] and generalizes the little Picard theorem. For proofs of the theorem of Borel, see also Nevanlinna [2], Fujimoto [1], Green [1]. Historically, Borel's theorem preceded and in fact motivated the work of Bloch and Cartan.

EXAMPLE 17. Let $Y$ be the complement of $n+k+1$ hyperplanes $H_{0}$, $H_{1}, \cdots, H_{n+k}$ in general position in $P_{n}(\boldsymbol{C})$. For each subset $I$ of $\{0,1, \cdots, n+k\}$ consisting of at least two but no more than $n+k-1$ indices, let $I^{\prime}$ be its complementary index set and let $\Delta_{I}\left(=\Delta_{I^{\prime}}\right)$ be the $(n-k)$ - 
dimensional linear subspace of $P_{n}(\boldsymbol{C})$ spanned by $\bigcap_{i \in I} H_{i}$ and $\bigcap_{j \in I^{\prime}} H_{j}$. We set $\Delta=\bigcup_{I} \Delta_{I}$. Then a simple combinatorial argument applied to Example 16 shows that $Y$ is complete hyperbolic modulo $\Delta$ and is hyperbolically imbedded in $P_{n}(\boldsymbol{C})$ modulo $\Delta$. It follows that every nonconstant holomorphic map $f: \boldsymbol{C}^{m} \rightarrow Y$ sends $\boldsymbol{C}^{m}$ into one of the $\Delta_{I}$ 's. If $k=n$, each $\Delta_{I}$ reduces to a point and it follows that the complement $Y$ of $2 n+1$ hyperplanes $H_{0}$, $H_{1}, \cdots, H_{2 n}$ in general position in $P_{n}(\boldsymbol{C})$ is complete hyperbolic and is hyperbolically imbedded in $P_{n}(\boldsymbol{C})$; see Dufresnoy [1], Fujimoto [1], [3], Kiernan and Kobayashi [2]. But a more careful combinatorial argument applied to the theorem of E. Borel yields the following theorem of Fujimoto [1] and Green [1].

THEOREM 12.1 Let $Y$ be the complement of $n+k+1$ hyperplanes in general position in $P_{n}(\boldsymbol{C})$. Then the image of any holomorphic map $f: \boldsymbol{C}^{m} \rightarrow Y$ is contained in a linear subspace of dimension $[n /(k+1)]$, the greatest integer $\leqq n /(k+1)$.

This bound is sharp, see the papers of Fujimoto and Green. Their theorem shows that if $Y$ is the complement of $n+2$ hyperplanes in general position in $P_{n}(\boldsymbol{C})$, then the image of $f: \boldsymbol{C}^{m} \rightarrow Y$ lies not merely in a diagonal hyperplane but in a linear subspace of dimension [ $n / 2]$. In connection with Example 16, we should note the result of Green [1] that if $Y$ is the complement of $n+2$ distinct hyperplanes in $P_{n}(\boldsymbol{C})$ (but not necessarily in general position), then the image of $f: \boldsymbol{C}^{m} \rightarrow Y$ lies in a hyperplane.

Although our principle of the great Picard theorem (Theorem 3.6) applies only to the case $k \geqq n$ in Example 17, Fujimoto [1] and Green [3] obtained generalizations of the great Picard theorem to all $k \geqq 1$. We quote only the result of Fujimoto. For the meromorphic version, see Green [3].

THEOREM 12.2. Let $X$ be a complex manifold and $A$ a complex subspace whose singularities are normal crossings. Let $Y$ be the complement of $n+k+1$ hyperplanes in general position in $P_{n}(\boldsymbol{C})$. Let $f: X-A \rightarrow Y$ be holomorphic. Then either the image $f(X-A)$ lies in an $(n-k)$-dimensional linear subspace of $P_{n}(\boldsymbol{C})$ or $f$ extends to a holomorphic map $f: X \rightarrow P_{n}(\boldsymbol{C})$.

There is another form of generalizing the great Picard theorem, which is probably more important in applications to algebraic geometry.

EXAMPLE 18. Let $M$ be a symmetric bounded domain and $\Gamma$ an arithmetically defined discontinuous group of automorphisms of $M$. Let $Y=\Gamma \backslash M$ and let $Z$ be the Satake-Baily-Borel compactification of $Y$. For a technical reason, assume that $\Gamma$ is acting freely on $M$. Then $Y$ is hyperbolically imbedded in $Z$ (see A. Borel [1], Kobayashi and Ochiai [2] and also Kiernan and Kobayashi [3] where the question concerning the Hausdorff property of Pyatetzki-Šapiro's compactfication has been resolved). Hence we can apply the principle of the great Picard theorem to this example. In the special case where $\boldsymbol{M}$ is the upper half-plane in $\boldsymbol{C}$ and $\Gamma$ is the principal congruence subgroup $\Gamma_{2}=\{A \in S L(2 ; Z) ; A=I \bmod 2\}$ of $S L(2 ; Z), Y=\Gamma \backslash M$ 
is $\boldsymbol{C}-\{0,1\}$ and $P_{1}(\boldsymbol{C})$ is the compactification of $Y$. (The covering projection $M \rightarrow \boldsymbol{C}-\{0,1\}$ is the modular function $\lambda$ mentioned in Example 4.)

In both Theorem 3.6 and Corollary 3.8 which are concerned with holomorphic extensions, we have to assume that the domain space $X$ is nonsingular as the following simple example shows. Let $Y$ be any compact hyperbolic space, e.g., an algebraic curve of genus greater than 1 , and let $X$ be the cone over $Y$. Let $A$ be the vertex of the cone. Then the natural projection $\pi: X-A \rightarrow Y$ does not extend to a holomorphic map of $X$ into $Y$. On the other hand, there is one important case where the holomorphic extension Theorem 3.6 holds for a singular $X$. Let $M$ be a symmetric bounded domain and $\Gamma$ an arithmetically defined discontinuous group of automorphisms of $M$. Let $X$ be the Satake-Baily-Borel compactification of $\Gamma \backslash M$ and let $A$ be the boundary of $\Gamma \backslash M$ added for the compactification so that $X=(\Gamma \backslash M) \cup A$. (Note that we switched the notation.) Let $Y$ be any hyperbolically imbedded complex space in another complex space $Z$. Then (Kiernan and Kobayashi [1])

THEOREM 12.3. Every holomorphic map $f: X-A \rightarrow Y$ extends to a holomorphic map $f: X \rightarrow Z$.

The following interesting example is due to Kiernan [3], [5].

Example 19. Let $Y=(C-\{0,1\}) \times(C-\{-1,1\})$. By Example 4 and Theorem $3.2, Y$ is complete hyperbolic. It is also hyperbolically imbedded in $P_{1}(C) \times P_{1}(C)$ in a natural manner. We shall now define two imbeddings of $Y$ into $P_{2}(\boldsymbol{C})$. Using a homogeneous coordinate system $u, v, w$ of $P_{2}(\boldsymbol{C})$, we define two open subsets $Y^{\prime}$ and $Y^{\prime \prime}$ of $P_{2}(C)$ by

$$
Y^{\prime}: \quad u \neq 0, v \neq 0, u \neq v, w \neq \pm u e^{v / u} ; \quad Y^{\prime \prime}: \quad u \neq 0, v \neq 0, u \neq v, v w \neq \pm u^{2} .
$$

Both of them are biholomorphic to $(\boldsymbol{C}-\{0,1\}) \times(\boldsymbol{C}-\{-1,1\})$ under the maps $\varphi: Y^{\prime} \rightarrow(\boldsymbol{C}-\{0,1\}) \times(\boldsymbol{C}-\{-1,1\}) \quad$ and $\quad \boldsymbol{\psi}: Y^{\prime \prime} \rightarrow(\boldsymbol{C}-\{0,1\}) \times(\boldsymbol{C}-\{-1,1\})$ defined by

$$
\varphi(u, v, w)=\left(v / u, e^{-u / v} w / u\right), \quad \psi(u, v, w)=\left(v / u, v w / u^{2}\right) .
$$

Neither $Y^{\prime}$ nor $Y^{\prime \prime}$ is hyperbolically imbedded in $P_{2}(\boldsymbol{C})$. In fact, the holomorphic map $f: D^{*} \rightarrow Y^{\prime}$ defined by $f(z)=\left(1, z, 2 e^{1 / z}\right)$ for $0<|z|<1$ has an essential singularity at $z=0$ and does not extend to a holomorphic map $f: D \rightarrow P_{2}(\boldsymbol{C})$. On the other hand, the holomorphic map $g: D^{*} \times D \rightarrow Y^{\prime \prime}$ defined by $g(z, t)=(1, z, t / z)$ has a pole at $(0,0)$ and does not extend to a holomorphic map $g: D \times D \rightarrow P_{2}(C)$, but it extends to a meromorphic map $g: D \times D \rightarrow P_{2}(C)$. The difference between $Y^{\prime}$ and $Y^{\prime \prime}$ lies in the fact that since $P_{2}(\boldsymbol{C})-Y^{\prime \prime}$ is a complex subspace of $P_{2}(\boldsymbol{C})$, the map $\psi$ extends to a bimeromorphic map $\psi: P_{2}(C) \rightarrow P_{1}(C) \times P_{1}(C)$.

We shall now combine Theorem 7.1, and Examples 13 and 15 in one general construction.

EXAMPLE 20. Let $Z$ be an $n$-dimensional projective algebraic manifold with canonical line bundle $K$. Let $S_{1}, \cdots, S_{k}$ be nonsingular divisors on $Z$ 
such that $S=S_{1}+\cdots+S_{k}$ has normal crossings. Let [S] denote the line bundle over $Z$ defined by the divisor $S$. If

$$
\limsup _{m \rightarrow \infty} \frac{1}{m^{n}} \operatorname{dim} \Gamma\left((K \cdot[S])^{m}\right)>0,
$$

then $Y=Z-S$ admits a pseudo-volume form $v_{Y}$ with negatively bounded Ricci form Ric $v_{Y}$. This includes the case where $S=0$ and $Z$ is of general type (see Theorem 7.1) and the case $K[S]$ is ample. The latter is the case considered by Carlson and Griffiths [1] and this generalization is due to Sakai [1]. The result of Carlson and Griffiths includes the case $Z=P_{n}(C)$ and $\operatorname{deg} S\left(=\sum \operatorname{deg} S_{i}\right) \geqq n+2$ such as Example 15. It includes also the case where $Z$ is an abelian variety and $S$ is ample. Leaving the detail to the papers of Carlson and Griffiths and Sakai, we describe the construction of $v_{Y}$. Let $H$ be any ample line bundle. Then there is a positive integer $m$ such that the line bundle $(K \cdot[S])^{m} H^{-1}$ admits a nontrivial section $\alpha$. We cover $Z$ by coordinate neighborhoods $\left\{U_{\lambda}\right\}$ with local coordinate systems $z_{\lambda}^{1}, \cdots, z_{\lambda}^{n}$. Let $\sigma_{i, \lambda}=0$ be the equation defining $U_{\lambda} \cap S_{i}$. We represent the section $\alpha$ and a hermitian fibre metric $h$ of $H$ by systems of locally defined functions $\left\{\alpha_{\lambda}\right\}$ and $\left\{h_{\lambda}\right\}$, respectively. Since $H$ is ample, we can choose $h$ such that $\left(\partial^{2} \log h_{\lambda} / \partial z_{\lambda}^{i} \partial \bar{z}_{\lambda}^{j}\right)$ is negative-definite. We define a pseudo-volume form $\omega_{\lambda}$ on $U_{\lambda}-S$ by

$$
\omega_{\lambda}=\frac{\left|\alpha_{\lambda}\right|^{2 / m}}{h_{\lambda}^{1 / m} \prod_{i}\left|\sigma_{i, \lambda}\right|^{2}} \prod_{j=1}^{n} \sqrt{ }-1 d z^{j} \wedge d \bar{z}^{j} .
$$

Then $\left\{\omega_{\lambda}\right\}$ defines a pseudo-volume form globally. Now the desired pseudovolume form $v_{Y}$ is given by

$$
v_{Y}=\frac{c}{\prod_{i}\left(\log \left\|\sigma_{i}\right\|^{2}\right)^{2}} \omega_{\lambda}
$$

where $\sigma_{i}$ is the global section of the line bundle $\left[S_{i}\right]$ defined by $\left\{\sigma_{i, \lambda}\right\}$, and $\left\|\sigma_{i}\right\|$ denotes its length with respect to any hermitian fibre metric in $\left[S_{i}\right]$. Taking positive constant $c$ sufficiently small, we obtain $v_{Y} \leqq\left(-\operatorname{Ric} v_{Y}\right)^{n}$. From the construction it is clear that $v_{Y}$ vanishes exactly at the zeroes of $\alpha$. In the special case where $K \cdot[S]$ is ample, we can take $H=K \cdot[S]$ and $\alpha=1$ so that $v_{Y}$ is positive everywhere. This volume form plays a central role in the equidimensional Nevanlinna theory (see Carlson and Griffiths [1]). The condition that $S_{1}, \cdots, S_{k}$ are nonsingular and $S=S_{1}+\cdots+S_{k}$ has normal crossings is essential; see Green [4] for counterexamples.

EXAMPLE 21. Let $G_{n, k}=U(n+k) / U(n) \times U(k)$ be the Grassmannian of $n$-planes in $\boldsymbol{C}^{n+k}$ and imbed $G_{n, k}$ in $P_{N}(\boldsymbol{C})\left(N=\left(\begin{array}{l}n \\ k\end{array}\right)-1\right)$ by Plücker coordinates. Let $H$ be the hyperplane line bundle of $P_{N}(C)$ restricted to $G_{n, k}$. If $K$ is the canonical line bundle of $G_{n, k}$, then $K=H^{-(n+k)}$. Let $H_{0}, H_{1}, \cdots, H_{n+k}$ be $n+k+1$ hyperplanes in $P_{N}(C)$ and let $Y=G_{n, k}-\left(\bigcup H_{i}\right)$. Assume that these hyperplanes are "in general position" in the sense that $S_{0}=H_{0} \cap G_{n, k}, \cdots, S_{n+k}=H_{n+k} \cap G_{n, k}$ are nonsingular and $S=S_{0}+\cdots+S_{n+k}$ has normal crossings, then $Y$ admits a volume form $v_{Y}$ with negatively 
bounded Ricci form and hence is meromorphically measure-hyperbolic; this follows immediately from Example 20. If we delete as many as $n k+2$ hyperplanes, much stronger results can be obtained (see Green [3]).

EXAMPLE 22. Let $X$ be a compact complex manifold immersed in a complex torus. Then $X$ is a principal torus bundle over a compact Kähler manifold of general type; see Matsushima [1], Yau [1], Ueno [1], [2]. If the fibre is nontrivial, then the intrinsic pseudo-volume form $\tilde{\Psi}_{X}$ vanishes identically by Example 2. If the fibre is trivial, then $X$ is of general type and hence meromorphically measure-hyperbolic by Theorem 7.1. We note that if one of the Chern numbers, e.g., the Euler number $c_{n}(X)$ or $\left(c_{1}(X)\right)^{n}$ of $X$ (with $\operatorname{dim} X=n$ ) is nonzero, then the fibre is trivial and $X$ is of general type.

One of the most interesting applications of the intrinsic distance is the following result of Royden [4], [5].

EXAMPLE 23. Let $T_{\mathrm{g}}$ denote the Teichmüller space of closed Riemann surfaces of genus $g \geqq 2$ (for a survey and references on Teichmüller spaces, see Bers [1]). It has a natural complex structure such that the cotangent space of $T_{\mathrm{g}}$ at $x \in T_{\mathrm{g}}$ is the space $Q_{x}$ of quadratic holomorphic differentials on the Riemann surface $W$ represented by $x$, i.e., $Q_{x}=\Gamma\left(K_{W}^{2}\right)$, where $K_{W}$ denotes the canonical line bundle of W. A norm $G(x, *)$ in $Q_{x}$ is defined by $G(x, \eta)=\int_{W}|\eta|$. Let $F(x, *)$ be the dual norm in the tangent space of $T_{\mathrm{g}}$ at $x$. The important theorem of Royden [4], [5] states that this norm $F$ coincides with the intrinsic differential metric $F_{T_{g}}$ of $T_{g}$ as a complex manifold. As a consequence, the intrinsic distance $d_{T_{\mathrm{g}}}$ coincides with the so-called Teichmüller distance on $T_{\mathrm{g}}$ introduced by Teichmüller before the natural complex structure was defined. Royden used his result to study automorphisms of $T_{\mathrm{g}}$. The theorem of Royden has been extended to Teichmüller spaces of punctured Riemann surfaces by Royden himself and also by Earle and Kra [1].

13. Unsolved problems. We list a number of open problems with some comments, classified into several groups. A few of them have been already mentioned in the main text.

(A) BASIC PROPERTIES OF INTRINSIC PSEUdo-DistANCES. Let $E_{X}$ and $F_{X}$ be the infinitesimal forms of $c_{X}$ and $d_{X}$ defined in $\$ 2$. The indicatrix $\Gamma_{x}$ of $E_{X}$ at $x \in X$ is given by

$$
\Gamma_{x}=\left\{v \in T_{x}(X) ; E_{X}(v)<1\right\} ;
$$

see Carathéodory [1], [2]. The indicatrix of $F_{X}$ at $x$ can be similarly defined.

Problem A.1. Study convexity of the indicatrix $\Gamma_{x}$ and differentiability of the boundary of $\Gamma_{x}$. For example, if $X$ is a bounded (pseudo-) convex domain with smooth boundary in $\boldsymbol{C}^{n}$, is the indicatrix also a convex domain with smooth boundary? Indicatrices are more intrinsic than the geometric shape of the domain $X$ in $\boldsymbol{C}^{n}$. If $X$ and $Y$ are two domains in $\boldsymbol{C}^{n}$ with a biholomorphic map $f: X \rightarrow Y$, then the indicatrix $\Gamma_{x} \subset T_{x}(X)$ is linearly biholomorphic to the indicatrix $\Gamma_{f(x)} \subset T_{f(x)}(Y)$ under the differential $f_{*}$. In $\boldsymbol{C}^{2}$ with coordinate system $z=x+i y, w=u+i v$, we consider a real ellipsoid

$$
X=X(a, b, c, d)=\left\{(z, w) ; a^{2} x^{2}+b^{2} y^{2}+c^{2} u^{2}+d^{2} v^{2}<1\right\}
$$


where $a, b, c, d$ are positive real numbers. Then $X(a, a, c, c)$ is a complex ellipsoid and is biholomorphic to the unit ball $X(1,1,1,1)$. What is the indicatrix $\Gamma_{0}$ of $X(a, b, c, d)$ at the origin? Is it the same as $X(a, b, c, d)$ under the obvious identification? If so, this implies immediately that $X(a, b, c, d)$ is biholomorphic to $X(1,1,1,1)$ only when $a=b, c=d$, the result proved by Webster recently by a completely different method. It is therefore of interest to determine the indicatrix for such a special domain as ellipsoid. If one wants to apply effectively methods of metric geometry of Busemann [1] and Rinow [1] to $\left(X, c_{X}\right)$ or $\left(X, d_{X}\right)$, it seems to be important to find out if the indicatrix is strictly convex.

Problem A.2. How smooth are $E_{X}$ and $F_{X}$ ? While $E_{X}$ is always continuous, $F_{X}$ need not be so. But Royden [1] has shown that $F_{X}$ is continuous if $X$ is taut (and hence if $X$ is complete hyperbolic). If $X$ is a bounded (pseudo-) convex domain in $C^{n}$ with smooth boundary, are $E_{X}$ and $F_{X}$ smooth on $T(X)$ outside the zero section? In order for methods of Finsler geometry to be applicable to $E_{X}$ and $F_{X}$, these intrinsic differential metrics must be at least twice differentiable outside the zero section of $T(X)$. For the question of differentiability of $E_{X}$, see Reiffen [1]. The question is clearly related to Problem A.1.

Problem A.3. Is $\log F_{X}$ plurisubharmonic on $T(X)$ ? From the very definition, $\log E_{X}$ is clearly plurisubharmonic. Geometrically, the question is whether $F_{X}$ has negative (or at least, nonpositive) "holomorphic sectional curvature." Masur [1] has shown that the Teichmüller space $T_{\mathrm{g}}, g \geqq 2$, does not have nonpositive "sectional curvature." But its holomorphic sectional curvature is probably negative in view of the fact that the Weil-Patterson metric has negative holomorphic sectional curvature (see Ahlfors [3]).

Problem A.4. For a symmetric bounded domain $X$, we have $c_{X}=d_{X}$; see Example 7. Is there any other domain for which $c_{X}=d_{X}$ ? For a Siegel domain $X$ of the first kind or, more generally, of the second kind, do we have $c_{X}=d_{X}$ ? For a real ellipsoid $X$ (see Problem A.1) do we have $c_{X}=d_{X}$ ? Earle [1] raises the question whether, for the Teichmüller space $T_{g}, c_{T_{g}}$ coincides with $d_{T_{\mathrm{g}}}$.

Problem A.5. Is $c_{X}$ an inner pseudo-distance? (See $\left.\$ 2.\right)^{4}$

Problem A.6. In connection with Theorem 4.1, we ask the following two questions:

(i) If a bounded domain $X$ is Cauchy-complete with respect to the Carathéodory distance $c_{X}$, is it finitely compact with respect to $c_{X}$ ? This is related to Problem A.5; if $c_{X}$ is inner, the answer to this question is affirmative.

(ii) If a bounded domain $X$ is a domain of holomorphy, is it complete with respect to $d_{X} ?^{5}$

Problem A.7. Results in $\S 4$ suggest that $c_{X}$ and the Bergman metric $d s_{X}^{2}$ behave in a similar manner. See also Graham [1]. For a bounded domain $X$,

\footnotetext{
${ }^{4} \mathrm{~T}$. Barth has shown me examples of $X$ for which $c_{X}$ is not inner.

${ }^{5} \mathrm{~T}$. Barth called my attention to N. Kerzman's example of a non-taut bounded domain of holomorphy (Notices Amer. Math. Soc. 16 (1969), 675-676).
} 
is there any relationship between "completeness with respect to $d s_{X}^{2}$ " and "completeness with respect to $c_{X}$ "? They seem to be more or less equivalent.

Problem A.8. Let $X$ be a compact complex space. According to Theorem 8.9, we can define an equivalence relation $R$ in $X$ using the class $\mathscr{C}_{7}$ of all compact hyperbolic spaces so that $X / R$ is compact hyperbolic. Define another equivalence relation $R^{\prime}$ on $X$ by

$$
x \sim_{R^{\prime}} x^{\prime} \quad \text { if and only if } d_{X}\left(x, x^{\prime}\right)=0 .
$$

Clearly, if $x$ and $x^{\prime}$ are $R^{\prime}$-equivalent, they are $R$-equivalent, i.e., there is a natural projection $X / R^{\prime} \rightarrow X / R$. Is $X / R^{\prime}$ a complex space in a natural manner? Equivalently, if $x$ and $x^{\prime}$ are $R$-equivalent, are they $R^{\prime}$-equivalent? To understand the $R^{\prime}$-equivalence relation, it is natural to examine the sets $Z(x)=\left\{x^{\prime} \in X ; d_{X}\left(x, x^{\prime}\right)=0\right\}$. Then each $Z(x)$ is connected. Let $\varepsilon$ be a positive number and $U_{\varepsilon}$ the $\varepsilon$-neighborhood of $Z(x)$ defined by $U_{\varepsilon}=\left\{x^{\prime} \in\right.$ $\left.X ; d_{X}\left(x, x^{\prime}\right)<\varepsilon\right\}$. In spite of the fact that $d_{U_{e}} \geqq d_{X}$ on $U_{\varepsilon}$, we still have $Z(x)=\left\{x^{\prime} \in U_{\varepsilon} ; d_{U_{\varepsilon}}\left(x, x^{\prime}\right)=0\right\}$, which shows that $Z(x)$ can be determined "locally" in a sense. The infinitesimal object corresponding to $Z(x)$ is given by $T_{x}^{0}=\left\{v \in T_{x}(X) ; F_{X}(v)=0\right\}$. We can ask if $Z(x)$ is a complex space with tangent space $T_{x}^{0}$ at $x$. Perhaps, one should examine Fermat varieties (see Example 14) with respect to these questions. A more modest question is the following: If $d_{\mathrm{X}}$ is not identically zero, does $X / R$ consist of more than one point? Let $X$ be a fibre space over $B$ with fibre $F$ such that $d_{B}=0$ and $d_{F}=0$. It is easy to see that $X / R$ is trivial (i.e., reduces to a single point), but it is not clear if $d_{X}=0$.

(B) BASIC PROPERTIES OF INTRINSIC PSEUDO-VOLUME FORMS.

Problem B.1. If we express the intrinsic pseudo-volume forms $\Phi_{X}, \Psi_{X}$ and $\tilde{\Psi}_{X}$ introduced in $\$ 5$ as

$$
\begin{gathered}
\Phi_{X}=E \Pi\left(\sqrt{ }-1 d z^{j} \wedge d \bar{z}^{j}\right), \quad \Psi_{X}=F \Pi\left(\sqrt{ }-1 d z^{j} \wedge d \bar{z}^{j}\right), \\
\tilde{\Psi}_{X}=\tilde{F} \Pi\left(\sqrt{ }-1 d z^{j} \wedge d \bar{z}^{j}\right),
\end{gathered}
$$

in terms of a local coordinate system $z^{1}, \cdots, z^{n}$ of $X$, we can ask questions similar to those of Problems A.2 and A.3. How smooth are the functions E, $F$ and $\tilde{F}$ ? ( $E$ is always continuous while $F$ and $\tilde{F}$ are upper semicontinuous.) Are $\log F$ and $\log \tilde{F}$ plurisubharmonic? ( $\log E$ is plurisubharmonic.)

Problem B.2. If $X$ and $Y$ are two complex manifolds, then

$$
\Phi_{X \times Y} \geqq \Phi_{X} \wedge \Phi_{Y}, \quad \Psi_{X \times Y} \geqq \Psi_{X} \wedge \Psi_{Y}, \quad \tilde{\Psi}_{X \times Y} \geqq \tilde{\Psi}_{X} \wedge \tilde{\Psi}_{Y},
$$

(where the projections of $X \times Y$ to $X$ and $Y$ are omitted to simplify the notation). Do we have the equality? For the Bergman kernel form, we have $B_{X \times Y}=B_{X} \wedge B_{Y}$ (Bremermann [1], Kobayashi [6]).

Problem B.3. Do we have $\Phi_{X} \leqq B_{X} \leqq \Psi_{X}$ in general or for a certain class of complex manifolds $X$ ? (Of course, we may have to multiply $B_{X}$ by a universal constant which depends only on the dimension of $X$.) When $X^{\prime}$ is a subdomain of $X$, we have $B_{X^{\prime}} \geqq B_{X}$ on $X^{\prime}$. So this is not an unreasonable question. For a bounded homogeneous domain $X$, we have $\Phi_{X}=B_{X}=\Psi_{X}$ 
(with the reservation that $B_{X}$ may have to be multiplied by a constant). For Siegel domains of the first or second kind and for real ellipsoid (see Problem A.1), what relations do we find for $\Phi_{X}, B_{X}$ and $\Psi_{Y}$ ?

Problem B.4. In view of Theorems 2.4 and 5.2 we ask: If $X$ is a ball in $\boldsymbol{C}^{n}$ with center at 0 , does $\Psi_{X-\{0\}}$ coincide with $\Psi_{X}$ on $X-\{0\}$ ?

Problem B.5. Blow up a point of the polydisk $D^{n}$ and denote the resulting space by $X$. Then we should have an example of space for which $\tilde{\Psi}_{X}$ differs from $\Psi_{X}$. But we can still ask if a (compact) measure-hyperbolic space is necessarily meromorphically measure-hyperbolic.

Problem B.6. If $\Psi_{\mathrm{X}}$ vanishes on a nonempty open subset of $X$, does it vanish identically?

(C) Differential geometric Questions.

Problem C.1. According to Yau [3], if $X$ is a complete Kähler manifold with Ricci tensor $\geqq 0$, then $c_{X}=0$ and $\Phi_{X}=0$ (Example 3$)$. Do we have $d_{X}=0$ and/or $\Psi_{X}=0$. (Assume, if necessary, that $X$ is compact or Ricci tensor 0 .)

Problem C.2. We do not know if a hyperbolic manifold admits a hermitian metric with negatively bounded holomorphic sectional curvature. Brody and Green have shown recently that hypersurfaces of large degree in $P_{n+1}(\boldsymbol{C})$ obtained by perturbing a Fermat variety are hyperbolic. One should find out if these hyperbolic manifolds admit hermitian metrics with negatively bounded holomorphic sectional curvature.

Problem C.3. Let $\tilde{X}$ be a covering space of a complex manifold $X$. In view of Theorem 3.3, we ask the following question. Does $X$ admit a hermitian metric with negatively bounded holomorphic sectional curvature if $\tilde{X}$ admits such a metric? According to $\mathrm{Wu}[\mathbf{1}]$, the answer is affirmative if $\tilde{X}$ is a finitely sheeted covering space of $X$. We can ask a similar question about volume forms with negatively bounded Ricci form.

Problem C.4. Let $X$ be a hermitian manifold with negatively bounded holomorphic sectional curvature. Does $X$ admit a volume form with negatively bounded Ricci form? (The converse is, of course, not true; consider, for example, a Fermat variety of large degree.)

The following question is not directly related to the main text of this paper but is of geometric interest.

Problem C.5. By the famous theorem of Remmert, every Stein manifold $X$ can be imbedded into some $\boldsymbol{C}^{N}$ as a closed complex submanifold. The natural flat metric of $\boldsymbol{C}^{N}$ induces on $X$ a complete Kähler metric with nonpositive holomorphic bisectional curvature. If a simply connected complex manifold $X$ admits a complete Kähler metric with nonpositive holomorphic bisectional curvature, is it a Stein manifold?

Problem C.6. Let $X$ be a compact complex manifold admitting a volume form $v_{X}$ with Ric $v_{X}<0$ (or $>0$ ). Does $X$ admit a Kähler metric with negative (or positive) Ricci tensor? (This is a special case of the Calabi conjecture [1].) Even for hypersurfaces in $P_{n+1}(C)$ this seems to be unknown.

(D) HOLOMORPHIC AND MEROMORPHIC MAPS.

Problem D.1. In connection with Theorem 8.6, we ask the following three questions: 
(i) Is every taut complex space complete hyperbolic? ${ }^{6}$

(ii) If $Y$ is hyperbolically imbedded in $Z$ modulo $\Delta$, is it tautly imbedded in $Z$ modulo $\Delta$ ?

(iii) Is there any implication between "taut $\bmod \Delta$ " and "complete hyperbolic mod $\Delta$ "? (See the footnote for (i).)

In (ii) and (iii) we might as well assume that $\Delta$ is a complex subspace of $Z$ since that is the only interesting case.

Problem D.2. Generalize Theorems 8.10 and 8.11 from C-hyperbolic spaces to hyperbolic spaces.

The following problem was posed by Lang [2] (see Theorems 8.12 and 8.13).

Problem D.3. Let $X$ be a compact complex space and $Y$ a compact hyperbolic space. Prove that there are only finitely many surjective holomorphic maps of $X$ onto $Y$. (This will imply also that there are only finitely many surjective meromorphic maps since $Y$ is compact hyperbolic.) A related question is whether Theorem 8.13 extends to (meromorphically) measure-hyperbolic spaces. It is also an important problem to show that there is an upper bound on the number of surjective morphisms which depends only on $X$ as in Theorem 8.12.

Problem D.4. In Example 14 we stated that the complement $Y$ of $n+2$ hyperplanes in general position in $P_{n}(C)$ is tautly imbedded in $P_{n}(C)$ modulo the diagonal hyperplanes and that this is a consequence of the main theorem of H. Cartan [1]. The result of Cartan is a little too long to be quoted here but it seems to imply much more than we have concluded. So the problem is to find a geometric statement which reflects the full strength of Cartan's theorem (cf. Kiernan and Kobayashi [2]). Since the proof of Cartan's theorem is long and difficult, it is desirable to find a simpler, geometric proof estimating the intrinsic pseudo-distance $d_{\mathrm{Y}}$. Cowen [2] has given such an estimate for the complement of $2^{n}+1$ hyperplanes in general position.

Problem D.5. Let $Y$ be hyperbolically imbedded in $Z$ modulo $\Delta$. The principle of the great Picard theorem (Theorem 3.6) has been so far proved only when $\Delta$ is empty. Extend this theorem to the case $\Delta$ is nonempty or at least $\Delta$ is a complex subspace so that theorems such as Theorem 12.2 of Fujimoto and Green can be derived from the general principle.

Problem D.6. Extend Theorem 10.4 to the case where $Y$ is a compact (meromorphically) measure-hyperbolic space. Find also a concept of complex space $Y$ measure-hyperbolically imbedded in $Z$ such that every equidimensional nondegenerate holomorphic (or meromorphic) map $f: X-A \rightarrow Y$ extends to $f: X \rightarrow Z$. The concept should include the situation where $Y$ is the complement of $n+2$ hyperplanes in general position in $Z=P_{n}(C)$.

Problem D.7. "Let $Y$ be a projective algebraic manifold whose irregularity $\left(=\operatorname{dim} H^{0,1}(Y ; \boldsymbol{C})\right)$ is greater than the dimension of $Y$. Then every holomorphic map $f: \boldsymbol{C} \rightarrow Y$ has its image in a proper closed subvariety of $Y$."

${ }^{6}$ No, for some reducible spaces according to T. Barth. 
This assertion was made by Bloch [2] without a complete proof. Recently, Ochiai [2] verified Bloch's assertion in many important cases. But the statement in its full generality still remains a conjecture. Let $A$ be the Albanese variety of $Y$ with canonical map $j: Y \rightarrow A$. If $j \circ f: C \rightarrow A$ has its image $j(f(\boldsymbol{C}))$ in a proper abelian subvariety, then $f(\boldsymbol{C})$ lies in a proper subvariety of $Y$.

Hence, Problem D.7 is related to the following:

Problem D.8. Let $X$ be a subvariety in a complex torus T. Let $x, x^{\prime} \in X$. Is it true then that $d_{X}\left(x, x^{\prime}\right)=0$ if and only if there is a complex subtorus $T^{\prime} \subset X$ containing $x$ and $x^{\prime}$. In view of Example 22, it suffices to consider the case where $X$ is of general type. Since the holomorphic bisectional curvature of $X$ (with respect to the metric coming from a flat metric of $T$ ) is nonpositive by the equations of Gauss and Codazzi, complex submanifolds of $T$ are good candidates for hyperbolic manifolds. ${ }^{7}$

Problem D.9. Let $T$ be a complex torus and $S=S_{1}+\cdots+S_{k}$ an ample divisor of $T$, where $S_{1}, \cdots, S_{k}$ are nonsingular and $S$ has normal crossings. Let $Y=T-S$. Is $Y$ hyperbolically imbedded in $T$ ? According to Example 20, $Y$ admits a volume form with negatively bounded Ricci form and is (meromorphically) measure-hyperbolic. We do not even know if $Y$ is hyperbolic or not. The following more modest question is still open (Griffiths [4]): Is every holomorphic map $f: \boldsymbol{C} \rightarrow Y$ constant? Special cases have been considered by Ax [1] and Ochiai [2].

(E) HOLOMORPHIC AND MEROMORPHIC AUTOMORPHISMS.

Problem E.1. Let $X$ be a complex space such that the complement of some compact subset $K$ is hyperbolic. Does this imply that $\operatorname{Aut}(X)$ is a Lie group? The affirmative answer would unify Theorems 9.1 and 9.2. A supporting evidence is that Fujimoto [4] has shown that $\operatorname{Aut}(X)$ is a Lie group when $X-K$ satisfies a condition closely related to the $C$ hyperbolicity.

Another possible generalization of Theorem 9.1 is to complex spaces which are hyperbolic modulo "small" subsets.

Problem E.2. Let $X$ be a complex space and

$$
\Delta=\left\{x \in X ; d_{X}(x, y)=0 \text { for some point } y \neq x\right\} .
$$

Then $X$ is hyperbolic modulo $\Delta$. If $\Delta$ is "small" in a suitable sense, is $\operatorname{Aut}(X)$ a Lie group? If $X-\Delta$ is connected, the natural restriction homomorphism $\operatorname{Aut}(X) \rightarrow \operatorname{Aut}(X-\Delta)$ is injective. Since $X-\Delta$ is hyperbolic and $\operatorname{Aut}(X-\Delta)$ is a Lie group, the question is whether the image of $\operatorname{Aut}(X)$ in $\operatorname{Aut}(X-\Delta)$ is closed or not. This is related to extension problems.

Somewhat related is the following question.

Problem E.3. Let $X$ be a measure-hyperbolic space. We know (Theorem 9.6) that no complex Lie groups act on $X$. Is $\operatorname{Aut}(X)$ a Lie group? Consider the Ricci form associated to the intrinsic pseudo-volume form $\Psi_{X}$. (Since $\Psi_{X}$ may not be smooth, this should be taken in the distributional sense.) If the

\footnotetext{
${ }^{7}$ This question has been settled recently by M. Green.
} 
Ricci form is positive definite in some open set, it is reasonable to expect $\operatorname{Aut}(X)$ to be a Lie group. So the problem is related to Problem B.1.

Problem E.4. Let $X$ be compact measure-hyperbolic. Is $\operatorname{Aut}(X)$ finite? We know that it is discrete (Theorem 9.7) and that it is finite if $X$ is of general type (Theorem 9.8). There are examples of nonsingular quartic surfaces in $P_{3}(\boldsymbol{C})$ (due to Fano and Severi) with discrete but infinite automorphism group (see Matsumura and Monsky [1]). But I rather doubt that they are measure-hyperbolic.

Problem E.5. Is Theorem 9.3 valid for a noncomplete hyperbolic space?

Problem E.6. Let $X$ be a hyperbolic space with finite total volume (measured either in terms of $\Psi_{X}$ or the top-dimensional Hausdorff measure defined by $\left.d_{X}\right)$. Is $\operatorname{Aut}(X)$ finite? The reasoning in Avérous and Kobayashi [1] shows that $\operatorname{Aut}(X)$ is compact.

Problem E.7. Is Theorem 9.11 valid for a noncompact $X$ with $\operatorname{Vol}(X)<\infty$ ?

Problem E.8. Is Theorem 9.12 valid for a compact complex manifold of general type?

The following problem has been studied by Eisenman [3] and Kulle [2].

Problem E.9. If $f$ is a proper holomorphic map of the unit ball in $\boldsymbol{C}^{n}$, $n \geqq 2$, into itself, is it an automorphism of the ball?

(F) Algebraic geOmetric Questions.

Problem F.1. Let $X$ be a compact complex space of dimension $n$. I conjecture that if $X$ is not of general type, i.e., if the Kodaira dimension $\kappa(X)$ is less than $n$, then $\tilde{\Psi}_{X}=0$. A little more modest conjecture is the following.

Problem F.2. Let $X$ be a compact complex manifold with canonical line bundle $K$. If $K>0$ (ample) or $K=0$, then $\tilde{\Psi}_{X}$ is most likely to be trivial. (The assumption implies $\kappa(X)<0$.) This problem is related to Problem C.1. If $K>0$, there exists a positive integer $m$ such that $K^{-m}$ is very ample. Let $\xi$ be a nontrivial section of $K^{-m}$. Then $\left\langle\tilde{\Psi}_{X}^{m}, \xi\right\rangle$ is an upper semicontinuous function on $X$. If the answer to Problem B.1 is affirmative so that $\log \tilde{F}$ is plurisubharmonic, then $\log \left\langle\tilde{\Psi}_{\mathrm{X}}^{m}, \xi\right\rangle$ is also plurisubharmonic and hence constant. Since $\xi$ vanishes at some point, this would imply $\tilde{\Psi}_{X}=0$. A special case of this problem is given by

Problem F.3. Let $X$ be a nonsingular hypersurface of $P_{n+1}(\boldsymbol{C})$. Let $d$ be the degree of $X$. The problem is to show that $\tilde{\Psi}_{X}=0$ if $d \leqq n+2$. If $d=2$, then $d_{X}=0$ and $\Psi_{X}=0$ (Examples 1 and 2). If $d=3$, then $X$ is unirational (see, for instance, Manin [1]) and we have $d_{X}=0$ and $\tilde{\Psi}_{X}=0$ (Examples 1 and 2). So the first nontrivial case is when $X$ is a quartic in $P_{3}(\boldsymbol{C})$. The Fermat quartic in $P_{3}(\boldsymbol{C})$ is a Kummer surface (see Shioda [1]) and $\tilde{\Psi}_{X}=0$ in this case (Example 2). Quartic surfaces and Kummer surfaces are all K3 surfaces. So we can ask if $\tilde{\Psi}_{\mathrm{X}}=0$ for any $K 3$ surface.

We shall now examine $d_{X}$ and $\tilde{\Psi}_{X}$ for compact complex manifolds $X$ of dimension 2 (called surfaces for simplicity's sake) using Kodaira's classification of surfaces. Without loss of generality, we may assume that $X$ is free of exceptional curves. Then there are seven classes (Kodaira [2], [4]): 
(I) The class of projective plane and ruled surfaces. These are not hyperbolic and $\tilde{\Psi}_{X}=0$.

(II) The class of K3 surfaces. This class contains surfaces without meromorphic functions as well as Kummer surfaces and quartic surfaces. As we have already seen, $d_{X}=0$ and $\tilde{\Psi}_{X}=0$ if $X$ is a Kummer surface. On the other hand, for every integer $g \geqq 2$, there exists an algebraic $K 3$ surface $X$ containing a curve of genus $g$ but no curves of smaller genus (see Šafarevič [1]). This makes it difficult to prove the degeneracy of $d_{X}$ and $\tilde{\Psi}_{X}$.

(III) The class of complex tori. Clearly, $d_{X}=0$ and $\Psi_{X}=0$.

(IV) The class of minimal elliptic surfaces with even $b_{1}, P_{12}>0$ and $K \neq 0$. (Here, $K$ is the canonical line bundle of $X, P_{m}=\operatorname{dim} \Gamma\left(K^{m}\right)$, and $b_{1}=$ the first Betti number.) In this case, $X$ is not hyperbolic and $\tilde{\Psi}_{X}=0$ (see Example 2).

(V) The class of minimal algebraic surfaces with $P_{2}>0, c_{1}^{2}>0$. They are of general type and meromorphically measure-hyperbolic. Some of them are hyperbolic and some are not. Probably, $d_{x}$ is not identically zero.

(VI) The class of minimal elliptic surfaces with odd $b_{1}, P_{12}>0$. (These are, of course, not algebraic.) As in the class (IV), $X$ is not hyperbolic and $\tilde{\Psi}_{X}=0$.

(VII) The class of minimal surfaces with $b_{1}=1, P_{12}=0$. (Again nonalgebraic, of course.) This class contains Hopf surfaces and elliptic surfaces. Including the examples constructed by Inoue [1], the universal covering of a known surface in this class is either (i) $D \times \boldsymbol{C}$, (ii) $\boldsymbol{C}^{2}$, or (iii) $\boldsymbol{C}^{2}-\{0\}$. So, for all known examples of this class, we know that $X$ is not hyperbolic and $\tilde{\Psi}_{X}=0$.

This classification table shows that a better understanding of $\mathrm{K} 3$ surfaces is needed to solve problems such as

(a) whether every meromorphically measure-hyperbolic (algebraic) surface is of general type, or

(b) whether every hyperbolic or meromorphically measure-hyperbolic surface is algebraic.

The classification of surfaces by Kodaira is stable under deformations. So we ask the following question.

Problem F.4. Let $X$ be a holomorphic family of compact complex manifolds parametrized by the space $S$. Let $\pi: X \rightarrow S$ be the projection and denote $\pi^{-1}(s), s \in S$, by $X_{s}$.

(i) If $X_{o}$ is hyperbolic (resp. (meromorphically) measure-hyperbolic) at one point $o \in S$, does there exist a neighborhood $U$ of $o$ in $S$ such that $X_{s}$ is also hyperbolic (resp. (meromorphically) measure-hyperbolic) for $s \in U$ ?

Cowen [1] has shown that if $X_{o}$ admits a hermitian metric with negatively bounded holomorphic sectional curvature, then there is a neighborhood $U$ of $o$ such that $\pi^{-1}(U)$ admits such a hermitian metric. Hence, each $X_{s}$ for $s \in U$ admits such a hermitian metric. (This is a generalization of a similar theorem of Grauert and Reckziegel [1].) A result similar to that of Grauert, Reckziegel and Cowen can be proven for volume forms. If $X_{o}$ is of general type, so is $X_{s}$ for $s \in U$. These facts indicate that the answer to the question above is most likely to be affirmative. 
(ii) Let $\left\{s_{k}\right\}$ be a sequence of points in $S$ converging to the point $o \in S$. Assume, for each $k, X_{s_{k}}$ is hyperbolic modulo a proper subvariety $\Delta$ (resp. (meromorphically) measure-hyperbolic). Does $X_{o}$ have the same property?

In view of a recent example of compact hyperbolic surface in $P_{3}(C)$ by Brody and Green, we cannot make $\Delta$ empty. For some results in this direction, see Wright [1].

Problem F.5. Let $Z, S=S_{1}+\cdots+S_{k}$ be as in Example 20. Does there exist an algebraic manifold $\tilde{Z}$ of general type which is a branched covering of $Z$ branched over $S$ ? An affirmative answer to this would give a geometric construction of a volume form $v_{Y}$ free of calculations as in Example 20. In Example 15, we constructed such a covering space when $Z=P_{n}(C)$ and $S$ consists of $n+2$ hyperplanes in general position. When $Z=P_{n}(C)$ and $S$ is a nonsingular divisor of degree $d \geqq n+3$, Carlson [1] constructed such a covering space $\tilde{Z}$ as follows. Let $p\left(z^{0}, \cdots, z^{n}\right)$ be a homogeneous polynomial of degree $d$ defining $S$. Then the homogeneous polynomial $q\left(z^{0}, \cdots, z^{n}, z^{n+1}\right)=\left(z^{n+1}\right)^{d}-p\left(z^{0}, \cdots, z^{n}\right)$ of degree $d$ in $n+2$ variables defines a nonsingular hypersurface of degree $d$ in $P_{n+1}(\boldsymbol{C})$. The projection $\pi: \tilde{Z} \rightarrow P_{n}(\mathbf{C})$ is given by $\left(z^{0}, \cdots, z^{n}, z^{n+1}\right)=\left(z^{1}, \cdots, z^{n}\right)$.

Problem F.6. The so-called adjunction formula (see Kodaira [2]) states that if $S$ is a nonsingular divisor of a compact complex manifold $X$, then $K_{S}=\left(K_{X} \cdot[S]\right)_{s}$, where $K_{S}$ and $K_{X}$ denote the canonical line bundles of $S$ and $X$, respectively. If $K_{X} \cdot[S]$ is ample, then $X-S$ admits a volume form with negatively bounded Ricci form (see Example 20). In this case, $K_{\mathbf{S}}$ is also ample by the adjunction formula and $S$ also admits a volume form with negatively bounded Ricci form (Theorem 7.1). This suggests the following question. To what extent can we claim that $X-S$ is measure-hyperbolic if and only if $S$ is measure-hyperbolic?

\section{BIBLIOGRAPHY}

This bibliography should be supplemented by the bibliography of my monograph Hyperbolic manifolds and holomorphic mappings.

P. R. Ahern and R. Schneider

[1] Isometries of H, Duke Math. J. 42 (1975), 321-326.

L. V. Ahlfors

[1] An extension of Schwarz's lemma, Trans. Amer. Math. Soc. 43 (1938), 359-364.

[2] Conformal invariants, Topics in Geometric Function Theory, McGraw-Hill, New York, 1973.

[3] Curvature properties of Teichmüller's space, J. Analyse Math. 9 (1961/62), 161-176. MR 25 \#192.

A. Andreotti and W. Stoll

[1] Extension of holomorphic maps, Ann. of Math. (2) 72 (1960), 312-349. MR 23 \#A1056.

G. Avérous and S. Kobayashi

[1] On automorphisms of spaces of non-positive curvature with finite volume (to appear). J. Ax

[1] Some topics in differential algebraic geometry. II: On the zeros of theta functions, Amer. J. Math. 94 (1972), 1205-1213.

W. L. Baily, Jr. and A. Borel

[1] Compactification of arithmetic quotients of bounded symmetric domains, Ann. of Math. (2) 84 (1966), 442-528. MR 35 \#6870. 
T. J. Barth

[1] The Kobayashi distance induces the standard topology, Proc. Amer. Math. Soc. 35 (1972), 439-441. MR 46 \#5668.

[2] Taut and tight complex manifolds, Proc. Amer. Math. Soc. 24 (1970), 429-431. MR 40 \#5897.

[3] Normality domains for families of holomorphic maps, Math. Ann. 190 (1971), 293-297. MR 43 \#3486.

[4] Families of holomorphic maps into Riemann surfaces, Trans. Amer. Math. Soc. 207 (1975), 175-187.

\section{S. Bergman}

[1] Über die Kernfunktion eines Bereiches und ihre Verhalten am Rande, J. Reine Angew. Math. 169 (1933), 1-42; ibid. 172 (1935), 89-128.

L. Bers 300 .

[1] Uniformization, moduli, and Kleinian groups, Bull. London Math. Soc. 4 (1972), 257-

A. Bloch

[1] Sur les systèmes de fonctions holomorphes à variétés linéaires lacunaires, Ann. Sci. École Norm. Sup. 43 (1926), 309-362.

[2] Sur les systèmes de fonctions uniformes satisfaisant à l'équation d'une variété algébrique dont l'irregularité depasse la dimension, J. Math. Pures Appl. 5 (1926), 19-66.

S. Bochner and D. Montgomery

[1] Groups on analytic manifolds, Ann. of Math. (2) 48 (1947), 659-669. MR 9, 174.

[2] Groups of differentiable and real or complex analytic transformations, Ann. of Math. (2) 46 (1945), 685-694. MR 7, 241.

A. Borel

[1] Some metric properties of arithmetic quotients of symmetric spaces and an extension theorem, J. Differential Geometry 6 (1972), 543-560. MR 49 \#3220.

A. Borel and R. Narasimhan

[1] Uniqueness conditions for certain holomorphic mappings, Invent. Math. 2 (1967), 247255. MR 35 \#414.

E. Borel

[1] Sur les zéros des fonctions entières, Acta Math. 20 (1897), 357-396.

H.-J. Bremermann

[1] Holomorphic continuation of the kernel function and the Bergman metric in several complex variables, Lectures on Functions of a Complex Variable, Univ. of Michigan Press, Ann Arbor, Mich., 1955, pp. 349-383. MR 17, 529.

R. Brody

[1] Intrinsic metrics and measures on compact complex manifolds, Harvard Ph.D. thesis, 1975.

H. Busemann

[1] The geometry of geodesics, Academic Press, New York, 1955. MR 17, 779.

E. Calabi

[1] On Kähler manifolds with vanishing canonical class, Algebraic Geometry and Topology (Sympos. in Honor of S. Lefschetz), Princeton Univ. Press, Princeton, N.J., 1957, pp. 78-89. MR 19, 62 .

L. A. Campbell and R. H. Ogawa

[1] On preserving the pseudodistance, Nagoya Math. J. 57 (1975) 37-47.

C. Carathéodory

[1] Über das Schwarzsche Lemma bei analytischen Funktionen von zwei komplexen Veränderlichen, Math. Ann. 97 (1926), 76-98.

[2] Über die Geometrie der analytischen Abbildungen, die durch analytische Funktionen von zwei Veränderlichen vermittelt werden, Abh. Math. Sem. Univ. Hamburg 6 (1928), 96-145.

[3] Über die Abbildungen, die durch Systeme von analytische Funktionen von mehreren Veränderlichen erzeugt werden, Math. Z. 34 (1932), 758-792. 


\section{J. A. Carlson}

[1] Some degeneracy theorems for entire functions with values in an algebraic variety, Trans. Amer. Math. Soc. 168 (1972), 273-301. MR 45 \#5417.

J. A. Carlson and P. A. Griffiths

[1] A defect relation for equidimensional holomorphic mappings between algebraic varieties, Ann. of Math. (2) 95 (1972), 557-584. MR 47 \#497.

H. Cartan

[1] Sur les systèmes de fonctions holomorphes à variétés linéaires lacunaires et leurs applications, Ann. Sci. École Norm. Sup. 45 (1928), 255-346.

[2] Quotients of complex analytic spaces, Contributions to Function Theory (Internat. Colloq., Bombay, 1960), Tata Institute of Fundamental Research, Bombay, 1960, pp. 1-15. MR 25 \#3199.

[3] Sur les groupes de transformations analytiques, Actualités Sci. Indust., no. 198, Hermann, Paris, 1935.

[4] Sur les fonctions de plusieurs variables complexes. L'itération des transformations interieurs d'un domaine borne, Math. Z. 35 (1932), 760-773.

[5] Les fonctions de deux variables complexes et le problème de la représentation analytique, J. Math. Pures Appl. 10 (1931), 1-114.

[6] Les transformations du produit topologique de deux domaines bornés, Bull. Soc. Math. France 64 (1936), 37-48.

S.-S. Chen

[1] Carathéodory distance and convexity with respect to bounded holomorphic functions, Proc. Amer. Math. Soc. 39 (1972), 305-307; corrections, ibid. 45 (1974), 468. MR 47 \#5298.

S. S. Chern

[1] Complex analytic mappings of Riemann surfaces. I, Amer. J. Math. 82 (1960), 323-337. MR 22 \#5985.

[2] The integrated form of the first main theorem for complex analytic mappings in several complex variables, Ann. of Math. (2) 71 (1960), 536-551. MR 23 \#A3276.

[3] On holomorphic mappings of Hermitian manifolds of the same dimension, Proc. Sympos. Pure Math., vol. 11, Amer. Math. Soc., Providence, R.I., 1968, pp. 157-170. MR 38 \#2714.

[4] Holomorphic curves in the plane, Differential Geometry (In Honor of K. Yano), Kinokuniya, Tokyo, 1972, pp. 73-94.

S. S. Chern and S. I. Goldberg

[1] On the volume decreasing property of a class of real harmonic mappings, Amer. J. Math. 97 (1975), 133-147.

S. S. Chern, H. I. Levine and L. Nirenberg

[1] Intrinsic norms on a complex manifold, Global Analysis (Papers in Honor of K. Kodaira), Univ. of Tokyo Press, Tokyo, 1969, pp. 119-139. MR 40 \#8084.

M. J. Cowen

[1] Families of negatively curved Hermitian manifolds, Proc. Amer. Math. Soc. 39 (1973), 362-366. MR 47 \#3712.

[2] The Kobayashi metric on $P_{n}-\left(2^{n+1}\right)$ hyperplanes, Value Distribution Theory, part A, Dekker, New York, 1974, pp. 205-223.

M. J. Cowen and P. A. Griffiths

[1] Holomorphic curves and metrics of negative curvature, J. Analyse Math.

K. Diederich

[1] Das Randverhalten der Bergmanschen Kernfunktion und Metrik in streng pseudokonvexen Gebieten, Math. Ann. 187 (1970), 9-36. MR 41 \#7149.

\section{A. Dinghas}

[1] Ein $n$-dimensionales Analogon des Schwarz-Pickschen Flächensatzes für holomorphe Abbildungen der komplexen Einheitskugel in eine Kähler-Mannigfaltigkeit, Festschr. Gedächtnisfeier K. Weierstrass, Westdeutscher Verlag, Cologne, 1966, pp. 477-494. MR 34 \#2935. 
A. Douady

[1] Le problème des modules pour les sous-espaces analytiques compacts d'un espace analytique donné, Ann. Inst. Fourier (Grenoble) 16 (1966), fasc. 1, 1-95. MR 34 \#2940.

H. Dufresnoy

[1] Théorie nouvelle des familles complexes normales. Applications à l'étude des fonctions algébroïdes, Ann. Sci. École Norm. Sup. (3) 61 (1944), 1-44. MR 7, 289.

C. J. Earle

[1] On the Carathéodory metric in Teichmüller spaces, Discontinuous Groups and Riemann Surfaces, Ann. of Math. Studies, no. 79, Princeton Univ. Press, Princeton, N.J., 1974, pp. 99-104.

C. J. Earle and I. Kra

[1] On holomorphic mappings between Teichmüller spaces, Contributions to Analysis, Academic Press, New York, 1974, pp. 107-124.

A. Eastwood

[1] A propos des variétés hyperboliques complètes, C.R. Acad. Sci. Paris, 280 (1975), 1071-1074.

D. Eisenman (now Pelles)

[1] Intrinsic measures on complex manifolds and holomorphic mappings, Mem. Amer. Math. Soc. No. 96 (1970). MR 41 \#3807.

[2] Holomorphic mappings into tight manifolds, Bull. Amer. Math. Soc. 76 (1970), 46-48. MR 40 \#5895.

[3] Proper holomorphic self-maps of the unit ball, Math. Ann. 190 (1971), 298-305; corrections, ibid. 202 (1973), 135-136. MR 43 \#3501; erratum, 43, p. 1698.

H. Fujimoto

[1] Extensions of the big Picard's theorem, Tôhoku Math. J. (2) 24 (1972), 415-422. MR 48 \#11578.

[2] On holomorphic maps into a taut complex space, Nagoya Math. J. 46 (1972), 49-61. MR 46 \#9375.

[3] Families of holomorphic maps into the projective space omitting some hyperplanes, J. Math. Soc. Japan 25 (1973), 235-249. MR 48 \#6473.

[4] On the holomorphic automorphism groups of complex spaces, Nagoya Math. J. 33 (1968), 85-106. MR 39 \#3038.

[5] On meromorphic maps into the complex projective space, J. Math. Soc. Japan 26 (1974), 272-288.

[6] On families of meromorphic maps into the complex projective space, Nagoya Math. J. 54 (1974), 21-51.

I. Graham

[1] Boundary behavior of the Carathéodory and Kobayashi metrics on strongly pseudo-convex domains in $C^{n}$ with smooth boundary, Trans. Amer. Math. Soc. 207 (1975), 219-240.

H. Grauert and H. Reckziegel

[1] Hermitesche Metriken und normale Familien holomorpher Abbildungen, Math. Z. 89 (1965), 108-125. MR 33 \#2827.

H. Grauert and R. Remmert

[1] Plurisubharmonische Funktionen in komplexen Räumen, Math. Z. 65 (1956), 175-194. MR 18, 475.

[2] Komplexe Räume, Math. Ann. 136 (1958), 245-318. MR 21 \#2063.

M. L. Green

[1] Holomorphic maps into complex projective space omitting hyperplanes, Trans. Amer. Math. Soc. 169 (1972), 89-103. MR 46 \#7547.

[2] The complement of the dual of a plane curve and some new hyperbolic manifolds, Value Distribution Theory, part A, Dekker, New York, 1974, pp. 119-131.

[3] Some Picard theorems for holomorphic maps to algebraic varieties, Amer. J. Math. 97 (1975), 43-75. 
[4] Some examples and counter-examples in value distribution theory for several variables, Compositio Math. (to appear)

[5] Holomorphic mappings to Grassmannians of lines, Proc. Sympos. Pure Math. vol. 27, Part II, Amer. Math. Soc., Providence, R.I., 1975, pp. 27-31.

P. A. Griffiths

[1] Holomorphic mappings into canonical algebraic varieties, Ann. of Math. (2) 93 (1971), 439-458. MR 43 \#7668.

[2] Two theorems on extensions of holomorphic mappings, Invent. Math. 14 (1971), 27-62. MR 45 \#2202.

[3] Some remarks on Nevanlinna theory, Value Distribution Theory, part A, Dekker, New York, 1974, pp. 1-11.

[4] Holomorphic mappings: Survey of some results and discussion of open problems, Bull. Amer. Math. Soc. 78 (1972), 374-382. MR 45 \#3786.

[5] A Schottky-Landau theorem for holomorphic mappings in several complex variables, Symposia Mathematica, vol. 10 (Convegno Geom. Diff. INDAM, Rome), Academic Press, New York, pp. 229-243.

[6] Differential geometry and complex analysis, Proc. Sympos. Pure Math., vol. 27, part II, Amer. Math. Soc., Providence, R. I., 1975, pp. 43-64.

P. A. Griffiths and J. King

[1] Nevanlinna theory and holomorphic mappings between algebraic varieties, Acta Math. 130 (1973), 145-220.

A. Hirschowitz

[1] Domaines de Stein et fonctions holomorphes bornées, Math. Ann. 213 (1975), 185-193.

F. Hirzebruch

[1] Topological methods in algebraic geometry, 3rd ed., Die Grundlehren der math. Wissenschaften, Band 131, Springer-Verlag, New York, 1966. MR 34 \#2573.

H. Huber

[1] Über analytische Abbildungen Riemannscher Flächen in sich, Comment. Math. Helv. 27 (1953), 1-72.

S. Iitaka

[1] On D-dimensions of algebraic varieties, J. Math. Soc. Japan 23 (1971), 356-373. MR 44 \#2749.

[2] On algebraic varieties whose universal covering manifolds are complex affine 3-spaces. I, Number Theory, Algebraic Geometry and Commutative Algebra (In Honor of Y. Akizuki), Kinokuniya, Tokyo, 1973, pp. 147-167.

[3] Projective manifolds whose universal covering manifolds are $C^{3}$, Manifolds Tokyo 1973, pp. 353-356.

M. Inoue

[1] On surfaces of class $\mathrm{VII}_{0}$, Manifolds Tokyo, 1973, pp. 389-392.

W. Kaup

[1] Hyperbolische komplexe Räume, Ann. Inst. Fourier (Grenoble) 18 (1968), fasc. 2, 303-330 (1969). MR 39 \#7138.

[2] Holomorphe Abbildungen in hyperbolische komplexe Räume, Geometry of Homogeneous Bounded Domains (C.I.M.E., $3^{\circ}$ Ciclo, Urbino, 1967), Edizioni Cremonese, Rome, 1968, pp. 111-123. MR 39 \#478.

[3] Reelle Transformationsgruppen und invariante Metriken auf komplexen Räumen, Invent. Math. 3 (1967), 43-70. MR 35 \#6865.

[4] Infinitesimale Transformationsgruppen komplexer Räume, Math. Ann. 160 (1965), 72-92. MR 31 \#5988.

H. Kerner

[1] Über die Automorphismengruppen kompakter komplexer Räume, Arch. Math. 11 (1960), 282-288. MR 22 \#8533. 


\section{N. Kerzman}

[1] The Bergman kernel function. Differentiability at the boundary, Math. Ann. 195 (1972), 149-158. MR 45 \#3762.

P. J. Kiernan

[1] On the relations between taut, tight and hyperbolic manifolds, Bull. Amer. Math. Soc. 76 (1970), 49-51. MR 40 \#5896.

[2] Hyperbolically imbedded spaces and the big Picard theorem, Math. Ann. 204 (1973), 203-209.

[3] Extensions of holomorphic maps, Trans. Amer. Math. Soc. 172 (1972), 347-355. MR 47 \#7066.

[4] On the compactifications of arithmetic quotients of symmetric spaces, Bull. Amer. Math. Soc. 80 (1974), 109-110. MR 48 \#4357.

[5] Holomorphic extension theorems, Value Distribution Theory, part A, Dekker, New York, 1974, pp. 97-107.

[6] Meromorphic mappings into compact complex spaces of general type, AMS Summer Institute, 1975.

[7] Quasiconformal mappings and Schwarz's lemma, Trans. Amer. Math. Soc. 148 (1970), 185-197. MR 41 \#467.

P. J. Kiernan and S. Kobayashi

[1] Satake compactification and extension of holomorphic mappings, Invent. Math. 16 (1972), 237-248.

[2] Holomorphic mappings into projective space with lacunary hyperplanes, Nagoya Math. J. 50 (1973), 199-216. MR 48 \#4353.

[3] Comments on Satake compactification and the great Picard theorem, J. Math. Soc. Japan (to appear).

S. Kobayashi

[1] Volume elements, holomorphic mappings and Schwarz's lemma, Proc. Sympos. Pure Math., vol. 11, Amer. Math. Soc., Providence, R. I., 1968, pp. 253-260. MR 38 \#6100.

[2] Distance, holomorphic mappings and the Schwarz lemma, J. Math. Soc. Japan 19 (1967), 481-485. MR 36 \#1705.

[3] Invariant distances on complex manifolds and holomorphic mappings, J. Math. Soc. Japan 19 (1967), 460-480. MR 38 \#736.

[4] Hyperbolic manifolds and holomorphic mappings, Pure and Appl. Math., 2, Dekker, New York, 1970. MR 43 \#3503.

[5] Some remarks and questions concerning the intrinsic distance, Tôhoku Math. J. (2) 25 (1973), 481-486, MR 48 \#8856.

[6] Geometry of bounded domains, Trans. Amer. Math. Soc. 92 (1959), 267-290. MR 22 \#3017.

[7] Negative vector bundles and complex Finsler structures, Nagoya Math. J. 57 (1975), 153-166.

[8] Transformation groups in differential geometry, Ergebnisse der mathematik und ihrer Grenzgebiete, Band 70, Springer-Verlag, Berlin and New York, 1972.

[9] On the automorphism group of a certain class of algebraic manifolds, Tôhoku Math. J. (2) 11 (1959), 184-190. MR 22 \#3014.

[10] On hyperbolic complex spaces and extension problems, Manifolds Tokyo 1973, pp. 333-342.

[11] Some problems on intrinsic distances and measures, The Greek Math. Soc., C. Carathéodory Sympos., 1973, pp. 306-317.

S. Kobayashi and T. Ochiai

[1] On complex manifolds with positive tangent bundles, J. Math. Soc. Japan 22 (1970), 499-525. MR 43 \#1231.

[2] Satake compactification and the great Picard theorem, J. Math. Soc. Japan 23 (1971), 340-350. MR 45 \#5418.

[3] Mappings into compact complex manifolds with negative first Chern class, J. Math. Soc. Japan 23 (1971), 137-148. MR 44 \#5514. 
[4] Meromorphic mappings into compact complex manifolds of general type, Invent. Math. (to appear).

K. Kodaira

[1] On Kähler varieties of restricted type (an intrinsic characterization of algebraic varieties), Ann. of Math. (2) 60 (1954), 28-48. MR 16, 952.

[2] On compact complex analytic surfaces. I-III, Ann. of Math. (2) 71 (1960), 111-152; (2) 77 (1963), 563-626; (2) 78 (1963), 1-40. MR 24 \#A2396; 32 \#1730.

[3] Holomorphic mappings of polydiscs into compact complex manifolds, J. Differential Geometry 6 (1971/72), 33-46. MR 46 \#386.

[4] On the structure of compact complex analytic surfaces. I-IV, Amer. J. Math. 86 (1964), 751-798; 88 (1966), 682-721; 90 (1968), 55-83, 1048-1066. MR 32 \#4708; 34 \#5112; 37 \#3603; 39 \#473.

R.-D. Kulle

[1] Holomorphe Abbildungen beschränkter symmetrischer Gebiete, Nachr. Akad. Wiss. Göttingen II Math.-Phys. 1973, 191-196.

[2] Eigentliche, holomorphs Abbildungen der Kugel in sich, Abh. Math. Sem. Univ. Hamburg 41 (1974), 5-16.

M. H. Kwack

[1] Generalization of the big Picard theorem, Ann. of Math. (2) 90 (1969), 9-22. MR 39 \#4445.

[2] A Schwarz lemma for canonical algebraic manifolds, Proc. Amer. Math. Soc. 41 (1973), 219-222. MR 48 \#4354.

[3] Mappings into hyperbolic spaces, Bull. Amer. Math. Soc. 79 (1973), 695-697. MR 47 \#8908.

[4] Some classical theorems for holomorphic mappings into hyperbolic manifolds, Proc. Sympos. Pure Math., vol. 27, Amer. Math. Soc., Providence, R.I., 1975, pp. 99-104.

[5] Holomorphic mappings into complex spaces.

S. Lang

[1] Diophantine geometry, Interscience Tracts in Pure and Appl. Math., no. 11, Interscience, New York, 1962. MR 26 \#119.

[2] Higher dimensional diophantine problems, Bull. Amer. Math. Soc. 80 (1974), 779-787.

Ju. I. Manin

[1] Cubic forms. Algebra, geometry, arithmetic, "Nauka," Moscow, 1972; English transl., North-Holland Math. Library, vol. 4, North-Holland, Amsterdam; American Elsevier, New York, 1974.

H. Masur

[1] On the negative curvature of Teichmüller space, Univ. of Minnesota Ph.D. Thesis, 1974; The curvature of Teichmüller space, Lecture Notes in Math., vol. 400, Springer-Verlag, Berlin and New York, 1974, pp. 122-123.

H. Matsumura

[1] On algebraic groups of birational transformations, Atti Accad. Naz. Lincei Rend. Cl. Sci. Fis. Mat. Natur. (8) 34 (1963), 151-155. MR 28 \#3041.

H. Matsumura and P. Monsky

[1] On the automorphisms of hypersurfaces, J. Math. Kyoto Univ. 3 (1963/64), 347-361. MR 29 \#5819.

Y. Matsushima

[1] Holomorphic immersions of a complex Kähler manifold into complex tori, J. Differential Geometry 9 (1974), 309-328.

B. G. Moǐsezon

[1] On $n$-dimensional compact complex varieties with $n$ algebraically independent meromorphic functions. I-III, Izv. Akad. Nauk SSSR Ser. Mat. 30 (1966), 133-174, 345-386, 621-656; English transl., Amer. Math. Soc. Transl. (2) 63 (1967), 51-177. MR 35 \#7355a, b, c. 


\section{P. Montel}

[1] Leçons sur les familles normales de fonctions analytiques et leurs applications, GauthierVillars, Paris, 1927.

[2] Sur les familles des fonctions analytiques, qui admettent des valeurs exceptionnelles dans un domaine, Ann. Sci. École Norm. Sup. 23 (1912).

R. Narasimhan

[1] Introduction to the theory of analytic spaces, Lecture Notes in Math., no. 25, SpringerVerlag, Berlin and New York, 1966. MR 36 \#428.

R. Nevanlinna

[1] Analytic functions, Die Grundlehren der math. Wissenschaften, Band 162, SpringerVerlag, Berlin and New York, 1970. MR 43 \#5003.

[2] Le théorème de Picard-Borel et la théorie des fonctions méromorphes, Gauthier-Villars, Paris, 1939.

T. Ochiai

[1] Some remarks on the defect relation of holomorphic curves, Osaka J. Math. 11 (1974), 483-501.

[2] On holomorphic curves in algebraic varieties with ample irregularity (to appear).

D. A. Pelles (formerly Eisenman)

[1] Holomorphic maps which preserve intrinsic measure, Amer. J. Math. 97 (1975), 1-15.

Klaus Peters

[1] Über holomorphe und meromorphe Abbildungen gewisser kompakter Mannigfaltigkeiten, Arch. Math. 15 (1964), 222-231.

Konrad Peters

[1] Starheitssätze für Pridukte normierte Vektorraume endlicher Dimension und für Pridukte hyperbolischer komplexer Räume, Math. Ann. 208 (1974), 343-354.

E. Picard

[1] Sur une propriété des fonctions entières, C. R. Acad. Sci. Paris 88 (1879), 1024-1027.

[2] Mémoire sur les fonctions entières, Ann. Sci. École Norm. Sup. (2) 9 (1880), 145-166; Selecta, Paris, 1928, pp. 1-21.

I. I. PjateckiY-Sapiro

[1] Geometry of classical domains and theory of automorphic functions, Fizmatgiz, Moscow, 1961; French transl., Dunod, Paris, 1966; English transl., Math. and its Applications, vol. 8, Gordon and Breach, New York, 1969. MR 25 \#231; 33 \#5949; 40 \#5908.

[2] Arithmetic groups in complex domains, Uspehi Mat. Nauk 19 (1964), no. 6 (120), 93121 = Russian Math. Surveys 19 (1964), no. 6, 83-109. MR 32 \#7790.

H. Reckziegel

[1] Hyperbolische Räume und normale Familien holomorpher Abbildungen, Dissertation, Univ. Göttingen, 1967.

H.-J. Reiffen

[1] Die differentialgeometrischen Eigenschaften der invarianten Distanzfunktion von Carathéodory, Schr. Math. Inst. Univ. Münster No. 26 (1963). MR 28 \#1320.

[2] Die Carathéodorysche Distanz und ihre zugehörige Differentialmetrik, Math. Ann. 161 (1965), 315-324. MR 33 \#4325.

R. Remmert

[1] Holomorphe und meromorphe Abbildungen komplexer Räume, Math. Ann. 133 (1957), 328-370. MR 19, 1193.

W. Rinow

[1] Die innere Geometrie der metrischen Räume, Die Grundlehren der math. Wissenschaften, Band 105, Springer-Verlag, Berlin, 1961. MR 23 \#A1290.

R. M. Robinson

[1] A generalization of Picard's and related theorems, Duke Math. J. 5 (1939), 118-132. 


\section{H. L. Royden}

[1] Remarks on the Kobayashi metric, Several Complex Variables, II (Proc. Internat. Conf., Univ. of Maryland, College Park, Md., 1970), Lecture Notes in Math., vol. 185, SpringerVerlag, Berlin, 1971, pp. 125-137. MR 46 \#3826.

[2] The extension of regular holomorphic maps, Proc. Amer. Math. Soc. 43 (1974), 306-310. MR 49 \#629.

[3] Holomorphic fiber bundles with hyperbolic fiber, Proc. Amer. Math. Soc. 43 (1974), 311-312. MR 49 \#3229.

[4] Automorphisms and isometries of Teichmüller spaces, Advances in the Theory of Riemann Surfaces (Proc. Conf., Stony Brook, N.Y., 1969), Ann. of Math. Studies, no. 66, Princeton Univ. Press, Princeton, N.J., 1971, pp. 369-383. MR 44 \#5452.

[5] Metrics on Teichmüller spaces, Lecture Notes in Math., vol. 400, Springer-Verlag, Berlin and New York, 1974, pp. 71-78.

I. R. Šafarevič, et al.

[1] Algebraic surfaces, Trudy Mat. Inst. Steklov. 75 (1965)=Proc. Steklov Inst. Math. 75 (1965). MR 32 \#7557; 35 \#6685.

F. Sakai

[1] Degeneracy of holomorphic maps with ramification, Invent. Math. 16 (1974), 212-229.

[2] Defect relations and ramification, Proc. Japan Acad. Sci. 50 (1974), 723-728.

[3] Defect relations for equidimensional holomorphic maps (to appear).

P. Samuel

[1] Lectures on old and new results on algebraic curves, Lectures on Math., no. 36, Tata Institute of Fundamental Research, Bombay, 1966. MR 36 \#5140.

I. Satake

[1] On compactifications of the quotient spaces for arithmetically defined discontinuous groups, Ann. of Math. (2) 72 (1960), 555-580. MR 30 \#594.

B. Shiffman

[1] Extension of positive line bundles and meromorphic maps, Invent. Math. 15 (1972), 332-347.

[2] Extension of holomorphic maps into Hermitian manifolds, Math. Ann. 194 (1971), 249-258. MR 45 \#598.

[3] Applications of geometric measure theory to value-distribution theory for meromorphic maps, Value Distribution Theory, part A, Dekker, New York, 1974, pp. 63-95.

T. Shioda

[1] Algebraic cycles on certain K3 surfaces in characteristic p, Manifolds Tokyo 1973, pp. 357-364.

N. Sibony

[1] Prolongement des fonctions holomorphes bornées et métrique de Carathéodory, Invent. Math. 29 (1975), 205-230.

[2] Prolongement analytique des fonctions holomorphes bornées, Séminaire Lelong 1972/73, Lecture Notes in Math., vol. 410, Springer-Verlag, Berlin and New York, 1974, pp. 44-66.

Y. T. Siu

[1] Techniques of extension of analytic objects, Lecture Notes Pure and Appl. Math., no. 8, Dekker, New York, 1974.

K. Stein

[1] Fortsetzung holomorpher Korrespondenzen, Invent. Math. 6 (1968), 78-90. MR 38 \#1286.

W. Stoll

[1] Über meromorphe Abbildungen komplexer Räume. I, II, Math. Ann. 136 (1958), 201239, 393-429. MR 21 \#2061; \#2062.

[2] Value distribution of holomorphic maps into compact complex manifolds, Lecture Notes in Math., vol. 135, Springer-Verlag, Berlin and New York, 1970. MR 42 \#2040. 
Y. Suyama

[1] On the first main theorem of holomorphic mappings from $C^{2}$ into $Q_{n-1}(C)$, Osaka J. Math. 11 (1974), 425-449.

K. Ueno

[1] Classification of algebraic varieties, Manifolds Tokyo 1973, pp. 329-332.

[2] Classification of algebraic varieties. I, Compositio Math. 27 (1973), 277-342.

T. Urata

[1] On meromorphic mappings into taut complex analytic spaces, Nagoya Math. J. 50 (1973), 49-65.

H. Weyl

[1] Meromorphic functions and analytic curves, Ann. of Math. Studies, no. 12, Princeton Univ. Press, Princeton, N.J., 1943. MR 5, 94.

M. W. Wright

[1] The behavior of the differential Kobayashi pseudometric in deformations of complex manifolds, Ph.D. Thesis, Stanford University, Stanford, Calif., 1974.

H.-H. Wu

[1] A remark on holomorphic sectional curvature, Indiana Univ. Math. J. 22 (1972/73), 1103-1108. MR 47 \#4191.

[2] Normal families of holomorphic mappings, Acta Math. 119 (1967), 193-233. MR 37 \#468.

[3] The equidistribution theory of holomorphic curves, Ann. of Math. Studies, no. 64, Princeton Univ. Press, Princeton, N.J., 1970. MR 42 \#7951.

[4] Mappings of Riemann surfaces (Nevanlinna theory), Proc. Sympos. Pure Math., vol. 11, Amer. Math. Soc., Providence, R.I., 1968, pp. 480-532. MR 38 \#6053.

[5] Remarks on the first main theorem in equidistribution theory. I-IV, J. Differential Geometry 2 (1968), 197-202, 369-384; 3 (1969), 83-94, 433-446. MR 43 \#2247a, b, c, d. H.-H. Wu and R. E. Greene

[1] Curvature and complex analysis, Bull. Amer. Math. Soc. 77 (1971), 1045-1049. MR 44 \#473.

S. T. Yau

[1] On the curvature of compact hermitian manifolds, Invent. Math. 15 (1974), 213-239.

[2] Intrinsic measures of compact complex manifolds, Math. Ann. 212 (1975), 317-329.

[3] A general Schwarz lemma for Kähler manifolds, Amer. J. Math. (to appear).

Department of Mathematics, University of CAlifornia, Berkeley, California 94720 